\title{
Tamanho amostral para estimar a concentração de organismos em água de lastro: uma abordagem bayesiana
}

\author{
Eliardo Guimarães da Costa
}

\author{
TESE APRESENTADA \\ $\mathrm{AO}$ \\ Instituto de Matemática e Estatística \\ DA \\ Universidade de SÃo Paulo \\ PARA \\ OBTENÇÃO DO TÍTULO \\ $\mathrm{DE}$ \\ DOUTOR EM CIÊNCIAS \\ Programa: Estatística \\ Orientador: Prof. Dr. Julio da Motta Singer \\ Coorientador: Prof. Dr. Carlos Daniel Paulino
}

Durante o desenvolvimento deste trabalho o autor recebeu auxílio financeiro da CAPES e do CNPq

São Paulo, junho de 2017 


\section{Tamanho amostral para estimar a concentração de organismos em água de lastro: uma abordagem bayesiana}

Esta versão da tese contém as correções e alterações sugeridas pela Comissão Julgadora durante a defesa da versão original do trabalho, realizada em 05/06/2017. Uma cópia da versão original está disponível no Instituto de Matemática e Estatística da Universidade de São Paulo.

Comissão Julgadora:

- Prof. Dr. Julio da Motta Singer (orientador) - IME-USP

- Prof. Dr. Carlos Daniel Paulino (coorientador) - Universidade de Lisboa

- Prof. Dr. Rafael Bassi Stern - UFSCar

- Prof. Dr. Jorge Alberto Achcar - FMRP-USP

- Prof. Dr. Mario de Castro Andrade Filho - ICMC-USP 
"Eu sou apenas um rapaz latino-americano sem dinheiro no banco, sem parentes importantes e vindo de Fortaleza [...]" trecho adaptado da música 'Apenas Um Rapaz Latino-Americano' de Belchior

"Walk down Corifeu Ave to the sound of $M P B$, I'm alive $[\ldots] "$ trecho adaptado da música 'Nine Out Of Ten' de Caetano Veloso "[...] está provado, quem espera nunca alcança [...]" 'Bom Conselho' de Chico Buarque 


\section{Agradecimentos}

À minha amada esposa, Rachel Tarini, por todo o apoio e companheirismo tanto nos momentos difíceis como nos bons momentos; com você São Paulo têm sido muito melhor, pelas alegrias no cotidiano, pelos planos e sonhos compartilhados $(-\log 0)$.

Aos meus amados pais (Sônia e Sérgio) e irmãos (Eduardo e Sara), pelo carinho, pela educação, pelo incentivo, pelo apoio mesmo que de longe; e aos meus familiares que de alguma forma contribuíram para minha formação pessoal e profissional.

Ao meu orientador, Professor Julio Singer, pela oportunidade e orientação, pela paciência, pelo apoio profissional e pessoal durante todo o doutorado, pelos ensinamentos que com certeza contribuíram para eu me tornar um profissional melhor.

Ao meu coorientador, Professor Daniel Paulino, pela oportunidade e orientação, pelas discussões e ensinamentos bayesianos, fundamentais para esta tese.

Aos membros titulares da banca examinadora as sugestões e comentários sobre o trabalho, e também aos membros suplentes pela disposição em completar a composição da banca.

Aos professores do IME-USP, em ordem alfabética: Chang Chiann, Heleno Bolfarine, Lucia Barroso, Nelson Tanaka, Silvia Ferrari e Silvia Nagib, que contribuíram para minha formação durante o doutorado.

Aos amigos do IME-USP pelas discussões e aprendizado nas disciplinas, pelos cafés no salão de chá do IME, pelos finais de semana de estudo na USP, pelos momentos de descontração na "salinha do doutorado", pelas idas ao bandejão etc, dentre os quais destaco, em ordem alfabética: Agatha Rodrigues, Alejandro Morán, Alice Lemos, Aline Oliveira, Aline Tsuyuguchi, Ana Paula Zerbeto, Andressa Cerqueira, Antonio Marcos, Ariadne Nogueira, Artur Lemonte, Benigno Alves, Brian Melo, Bruno Monte, Bruno Santos, Daniel Reis, Diana Soto, Edite Taufer, Elivaldo Macedo, Eriton Santos, Elizabeth Patiño, Elisângela Rodrigues, Fabiana Barros, Fabio Oki, Guaraci Requena, Gilberto Sassi, Giovana Fumes, Guilherme Ost, Helton Graziadei, Hérica Priscila, Isabela Coelho, Isabelie Hernandez, João Nunes, Josemir Almeida, Kaique Matias, Leandro Correia, Leandro Ferreira, Lucas Reis, Luis Hernando Vanegas, Luz Marina Poveda, Marcio Diniz, Marcos Rafael, Mark Gannon, Mel Oliveira, Michel Helcias, Moisés Medeiros, Renato Ciani, Paloma Uribe, Tamy Tsujimoto, Tiago Maia, Tuany Castro, Vinicius Cokin, William Duran e Yolanda Olmos.

Aos funcionários da secretaria, dentre os quais destaco: Tamara Sciré e Regiane Guimarães, pelo atendimento e prontidão em resolver os problemas burocráticos; aos funcionários do salão de chá por nos servir com alegria este líquido precioso, o café!

Aos Professores Maurício e Juvêncio do DEMA-UFC e à Professora Socorro Oliveira (Matemática) 
do HDO por terem regado essa "semente do conhecimento" na graduação e no ensino médio, respectivamente.

Aos amigos da república Gobbo os momentos de descontração, dentre os quais destaco, em ordem alfabética: Benigno Alves, Carlos Eduardo, Felipe Viana, Licia Sales, Othon, Tom e Vinicius Queiroz.

Aos amigos da república na Eiras: Rodrigo Silva, Victor Tayra e Vinicius Cokin, pela acolhida na república e pelos momentos de descontração, churrascos etc.

Ao CNPq e à CAPES o apoio financeiro. 


\section{Resumo}

COSTA, E. G. Tamanho amostral para estimar a concentração de organismos em água de lastro: uma abordagem bayesiana. 2017. 82 f. Tese (Doutorado) - Instituto de Matemática e Estatística, Universidade de São Paulo, São Paulo.

Metodologias para obtenção do tamanho amostral para estimar a concentração de organismos em água de lastro e verificar normas internacionais são desenvolvidas sob uma abordagem bayesiana. Consideramos os critérios da cobertura média, do tamanho médio e da minimização do custo total sob os modelos Poisson com distribuição a priori gama e binomial negativo com distribuição a priori Pearson Tipo VI. Além disso, consideramos um processo Dirichlet como distribuição a priori no modelo Poisson com o propósito de obter maior flexibilidade e robustez. Para fins de aplicação, implementamos rotinas computacionais usando a linguagem $\mathrm{R}$.

Palavras-chave: critério da cobertura média, critério do comprimento médio, risco de Bayes, distribuição Poisson, distribuição binomial negativa, processo Dirichlet. 


\section{Abstract}

COSTA, E. G. Sample size for estimating the organism concentration in ballast water: a Bayesian approach. 2017. 82 pp. DSc thesis - Instituto de Matemática e Estatística, Universidade de São Paulo, São Paulo.

Sample size methodologies for estimating the organism concentration in ballast water and for verifying international standards are developed under a Bayesian approach. We consider the criteria of average coverage, of average length and of total cost minimization under the Poisson model with a gamma prior distribution and the negative binomial model with a Pearson type VI prior distribution. Furthermore, we consider a Dirichlet process as a prior distribution in the Poisson model with the purpose to gain more flexibility and robustness. For practical applications, we implemented computational routines using the $\mathrm{R}$ language.

Keywords: average coverage criterion, average length criterion, Bayes risk, Poisson distribution, negative binomial distribution, Dirichlet process. 


\section{Sumário}

Lista de Figuras $\quad$ xi

Lista de Tabelas $\quad$ xiii

1 Introdução 1

1.1 Abordagem por meio de teste de hipóteses . . . . . . . . . . . . . . 2

1.2 Abordagem por meio de intervalos de confiança . . . . . . . . . . . . . . . . 4

2 Modelos bayesianos $\quad 9$

2.1 Modelo Poisson com distribuição a priori gama . . . . . . . . . . . . . . . . 9

2.2 Modelo binomial negativo com distribuição a priori Pearson Tipo VI . . . . . . . . . 10

2.3 Modelo Poisson com distribuição a priori não-paramétrica . . . . . . . . . . . . . . . 13

3 Determinação do tamanho amostral sob o enfoque bayesiano 15

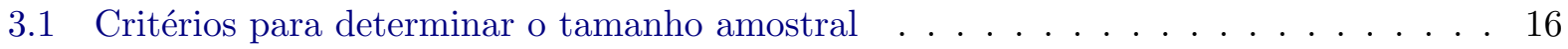

3.1 .1 Critério da cobertura média $(\mathrm{CCobM}) \ldots \ldots \ldots \ldots$

3.1 .2 Critério do comprimento médio $(\mathrm{CCompM}) \ldots \ldots \ldots \ldots$

3.1 .3 Minimização do custo total $(\mathrm{MCT}) \ldots \ldots \ldots \ldots$. . . . . . . . . . 19

4 Resultados e discussão $\quad \mathbf{2 5}$

4.1 Abordagem baseada nos critérios CCobM e CCompM . . . . . . . . . . . . 25

4.2 Abordagem baseada na Teoria da Decisão . . . . . . . . . . . . . . . . . . . 31

4.3 Representação da informação a priori . . . . . . . . . . . . . . . . . . . . 33

4.4 Considerações finais . . . . . . . . . . . . . . . . . 35

$\begin{array}{ll}\text { A Modelo Poisson com distribuição a priori gama } & 39\end{array}$

A.1 Distribuição a posteriori e propriedades . . . . . . . . . . . . . . . . . 39

A.2 Demonstração do Teorema $3.1 \ldots \ldots \ldots \ldots$. . . . . . . . . . . . . 40

A.3 Obtenção da decisão de Bayes usando MCT . . . . . . . . . . . . . . . . . . . . . 42

A.3.1 Função perda $1 \ldots \ldots \ldots \ldots$. . . . . . . . . . . . . . . 42

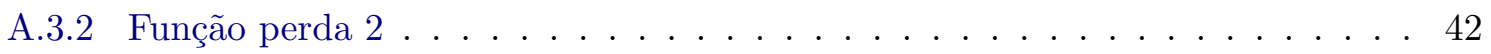

A.4 Algoritmos necessários para o cálculo do tamanho amostral . . . . . . . . . . . . 43

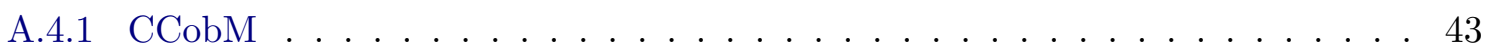

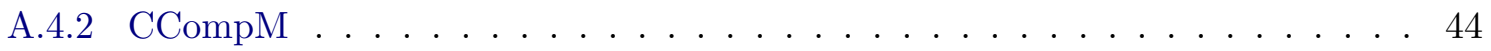

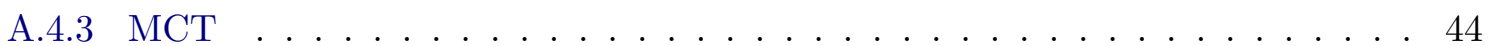


B Modelo binomial negativo com distribuição a priori Pearson Tipo VI 47

B.1 Distribuição a posteriori e propriedades . . . . . . . . . . . . . . 47

B.2 Obtenção da decisão de Bayes usando MCT . . . . . . . . . . . . . . . . . . . . 48

B.2.1 Função perda $1 \ldots \ldots \ldots \ldots \ldots \ldots$. . . . . . . . . . . . . . . . . . . . . . . . . . . .

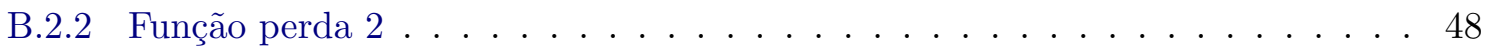

B.3 Algoritmos necessários para o cálculo do tamanho amostral . . . . . . . . . . . . 49

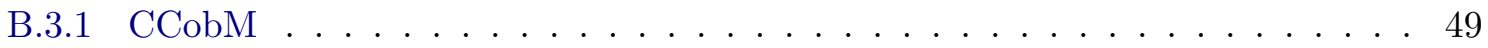

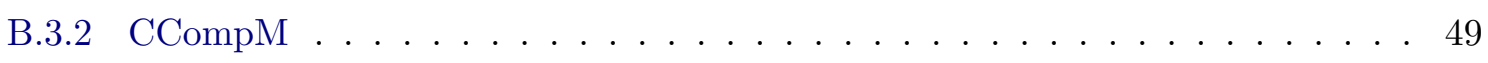

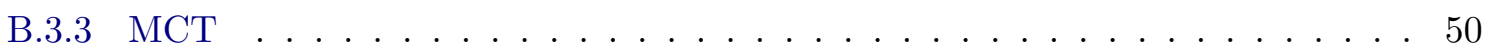

$\begin{array}{ll}\text { C Modelo Poisson com distribuição a priori não-paramétrica } & \mathbf{5 1}\end{array}$

C.1 Distribuição a posteriori e propriedades . . . . . . . . . . . . . 51

C.2 Obtenção da decisão de Bayes usando MCT . . . . . . . . . . . . . . . . . . 56

C.2.1 Função perda $3 \ldots \ldots \ldots \ldots \ldots \ldots$. . . . . . . . . . . . . . . . . . .

C.3 Algoritmos necessários para o cálculo do tamanho amostral . . . . . . . . . . . . 57

C.3.1 Distribuição $\nu\left(\cdot \mid \boldsymbol{x}_{n}\right) \ldots \ldots \ldots \ldots \ldots \ldots \ldots \ldots$

C.3.2 Estimação de $($ C.13) . . . . . . . . . . . . . . . . . . . 57

C.3.3 Obtenção da região HPD de probabilidade $1-\rho \ldots \ldots \ldots$. . . . . . . 58

C.3.4 Obtenção da região HPD de tamanho $\ell \ldots \ldots \ldots \ldots$. . . . . . . 58

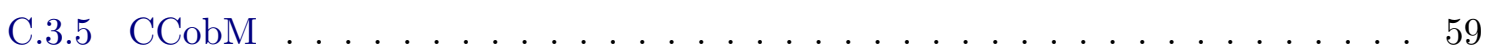

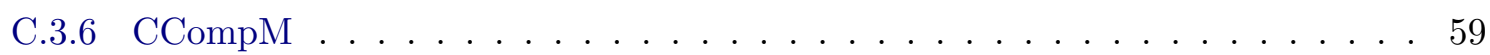

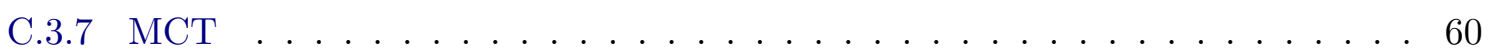

$\begin{array}{lll}\text { Referências } & 61\end{array}$

$\begin{array}{ll}\text { Índice Remissivo } & 66\end{array}$ 


\section{Lista de Figuras}

2.1 Funções densidade da distribuição $G\left(\theta_{0}, \theta_{0} / \lambda_{0}\right) \ldots \ldots \ldots \ldots \ldots \ldots$

2.2 Funções densidade da distribuição $P V I\left(0, \phi / w, \theta_{0}, \theta_{0} / \lambda_{0}+1\right) \operatorname{com} w=1, \lambda_{0}=3$ e

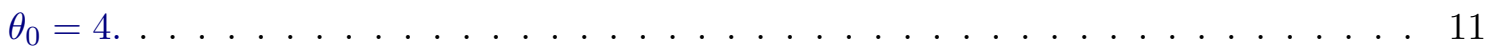

2.3 Funções densidade da distribuição $P V I\left(0, \phi / w, \theta_{0}, \theta_{0} / \lambda_{0}+1\right) \operatorname{com} w=1 . \quad \ldots \ldots 12$ 


\section{Lista de Tabelas}

4.1 Número mínimo de alíquotas $(n)$ com informação a priori $\lambda_{0}=10$ sob o modelo (2.1)-(2.2) com o critério CCobM $(3.1)$ e $\rho=0,05 \ldots \ldots \ldots \ldots$

4.2 Número mínimo de alíquotas $(n)$ com informação a priori $\lambda_{0}=10(w / \phi)$ sob o modelo (2.3)-(2.4) com o critério CCobM (3.1) e $\rho=0,05 \ldots \ldots \ldots \ldots \ldots$

4.3 Número mínimo de alíquotas $(n)$ usando o critério CCobM sob o modelo (2.5)-(2.7)

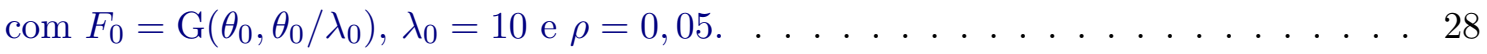

4.4 Número mínimo de alíquotas $(n)$ com informação a priori $\lambda_{0}=10$ sob o modelo (2.1)-(2.2) com o critério CCompM (3.3) e $\rho=0,05 \ldots \ldots \ldots \ldots$

4.5 Número mínimo de alíquotas $(n)$ com informação a priori $\lambda_{0}=10$ usando o Teorema 3.1 sob o modelo $(2.1)-(2.2)$ e $\rho=0,05 \ldots \ldots \ldots \ldots \ldots$

4.6 Número mínimo de alíquotas $(n)$ com informação a priori $\lambda_{0}=10(w / \phi)$ sob o modelo (2.3)-(2.4) com o critério CCompM (3.3) e $\rho=0,05 \ldots \ldots \ldots$. . . . . . . 29

4.7 Número mínimo de alíquotas $(n)$ usando o critério CCompM sob o modelo (2.5)-(2.7)

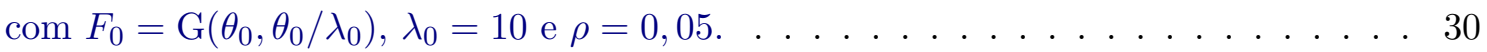

4.8 Número mínimo de alíquotas $(n)$ com informação a priori $\lambda_{0}=10$ sob o modelo (2.1)-(2.2) com a função perda 1 e $\rho=0,05 \ldots \ldots$. . . . . . . . . . . 33

4.9 Número mínimo de alíquotas $(n)$ com informação a priori $\lambda_{0}=10(w / \phi)$ sob o modelo (2.3)-(2.4) com a função perda 1 e $\rho=0,05 \ldots \ldots$. . . . . . . . . . 33

4.10 Número mínimo de alíquotas $(n)$ com informação a priori $\lambda_{0}=10$ sob o modelo

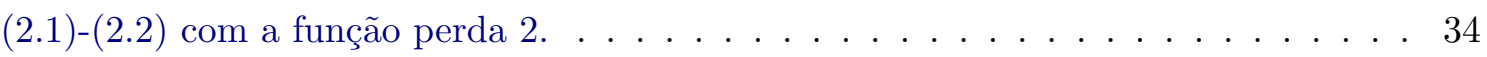

4.11 Número mínimo de alíquotas $(n)$ com informação a priori $\lambda_{0}=10(w / \phi)$ sob o modelo

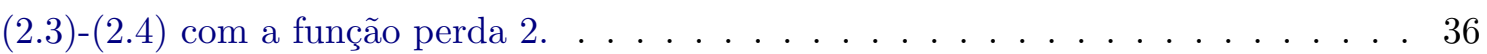

4.12 Número mínimo de alíquotas $(n)$ com informação a priori $\lambda_{0}=10$ sob o modelo (2.5)(2.7) com a função perda 3 e $W(d t)$ sendo a função densidade de uma distribuição normal com média e desvio padrão iguais a $10 \ldots \ldots$. . . . . . . . . . . . 37 


\section{Capítulo 1}

\section{Introdução}

Com o crescimento do tráfego marítimo e o uso de grandes navios, a água de lastro se tornou um dos principais agentes dispersivos de organismos nocivos (David et al., 2007; Souza et al., 2001). De acordo com Ruiz et al. (2000), patógenos humanos também estão sendo transportados através de água de lastro. Como exemplo da consequência desse transporte, podemos mencionar a epidemia de cólera ocorrida no Peru em 1991 e rapidamente disseminada pela América Latina via água de lastro (McCarthy et al., 1992).

A norma D-2 da International Convention for the Control and Management of Ship Ballast Water and Sediments, adotada pela International Maritime Organization (IMO) em 2004, estabelece limites superiores para a concentração média de organismos vivos em água de lastro descarregada por navios. Em particular, essa norma requer que a água de lastro descarregada por navios contenham menos que 10 organismos vivos com dimensão mínima $\geq 50 \mu \mathrm{m} / \mathrm{m}^{3}$.

Por causa de restrições de custo e tempo, examinar todo o volume descarregado não é praticável e amostragem deve ser considerada. Diferentes abordagens para esse problema têm sido consideradas na literatura. Basurko \& Mesbahi (2011) adotam a distribuição binomial, mas seus resultados sugerem que um grande volume de água de lastro deve ser amostrado para garantir o cumprimento da norma com precisão razoável. Miller et al. (2011) usaram a distribuição Poisson e sua abordagem produziu resultados mais factíveis, com volumes amostrais bem menores que o volume de água no tanque. Ambas as abordagens, entretanto, são baseadas na suposição de que a concentração de organismos é homogênea no tanque de lastro. Isto pode não ser razoável na prática, como apontado por Miller et al. (2011), Murphy et al. (2002) ou Carney et al. (2013), entre outros. Mais recentemente, Bierman et al. (2012) e Costa (2013), trabalhando independentemente, propuseram a utilização da distribuição binomial negativa que leva em conta a esperada heterogeneidade da 
concentração. Em todos os casos, problemas relacionados às implicações da "representatividade" estatística da amostra são discutidos, sem uma clara definição do que isso significa. Com relação ao cumprimento da norma D-2, uma "amostra representativa" é aquela na qual a análise permite a estimação da concentração de organismos vivos com uma precisão fixada previamente. Dado que essa precisão está diretamente relacionada com o modelo estatístico subjacente e que diferentes modelos são baseados em diferentes suposições sobre a distribuição dos organismos no tanque, a heterogeneidade desempenha um papel importante na busca pela representatividade estatística.

Costa et al. $(2015,2016)$ consideram modelos específicos para acomodar diferentes suposições sobre a heterogeneidade da concentração e obtêm valores limites, número mínimo de alíquotas (ou volume amostral) para determinar se um navio cumpre a norma D-2 sob diferentes esquemas amostrais e níveis de precisão. Usamos o termo "organismos" no lugar de "organismos vivos" ao longo do texto. O parâmetro que estamos interessados é a concentração média $\lambda$ de organismos por unidade de volume no tanque de água de lastro $\left(m^{3}\right.$ ou $m L$ no caso da norma D-2). Assim, podemos escrever

$$
\lambda=\frac{\mathrm{N}^{\mathrm{o}} \text { de organismos no tanque }}{\text { Volume de água no tanque }} .
$$

\subsection{Abordagem por meio de teste de hipóteses}

Em termos estatísticos, decidir se um navio cumpre ou não a norma D-2 é equivalente a testar a hipótese de que $\lambda \leq 10$ versus a hipótese alternativa de que $\lambda>10$ com base em uma amostra de $v m^{3}$ de água de lastro obtida de uma ou mais alíquotas coletadas durante o deslastre. Costa et al. (2015) abordam esse problema considerando diferentes suposições para a distribuição dos organismos no tanque. Esses autores começam supondo que essa distribuição é homogênea, em cujo caso uma distribuição Poisson pode ser adotado. Mais especificamente, rotulando por $X$ o número de organismos na amostra, a probabilidade de que $X=x$ é

$$
\mathbb{P}[X=x \mid \lambda]=\exp (-\lambda v)(\lambda v)^{x} / x !
$$

para $x=0,1, \ldots$. Se a concentração média de organismos no tanque de lastro é $\lambda$, então esperamos encontrar $v \lambda$ organismos em uma amostra com volume $v$. Sob esse modelo, a regra de decisão é indicar não cumprimento da norma D-2 se $X>c$, em que $c$ e o volume amostral mínimo $v$ são determinados de forma que

$$
\beta_{1}=\mathbb{P}[X>c \mid \lambda=10] \quad \text { e } \quad \beta_{2}\left(\lambda_{A}\right)=\mathbb{P}\left[X \leq c \mid \lambda=\lambda_{A}>10\right],
$$


em que $\beta_{1}$ denota a probabilidade do erro do Tipo I e $\beta_{2}\left(\lambda_{A}\right)$ a probabilidade do erro do Tipo II para um valor especificado $\lambda_{A}>10$, ou indicar cumprimento da norma em caso contrário. De preferência, $\beta_{1}$ e $\beta_{2}$ devem ser pequenos, mas isso pode demandar volumes amostrais muito grandes de maneira que sua escolha deve ser baseada em custos.

Note que se o volume amostrado $(v)$ é composto de $n$ alíquotas de volume $w(v=n w)$ e $X_{i}$ denota o número de organismos observados na $i$-ésima alíquota, então $X=\sum_{i=1}^{n} X_{i}$ e a regra de decisão é similar.

Na prática, a suposição de homogeneidade subjacente a distribuição Poisson é raramente sustentada. A distribuição dos organismos no tanque de lastro depende de sua configuração, do fluxo de deslastre, do local de onde a água é coletada, das condições de luminosidade, mudanças na densidade da água etc., como discutido em Murphy et al. (2002), entre outros. A determinação da distribuição exata é difícil, se não impossível. Para contornar esse problema, Costa et al. (2015) consideram diferentes configurações de heterogeneidade, identificam modelos apropriados e indicam como é a regra de decisão em cada caso.

Supondo que há alguma informação sobre a variação da concentração em diferentes partes do tanque, podemos considerar a concentração como uma variável aleatória $U$ seguindo alguma distribuição de probabilidade conveniente. Uma alternativa é supor que $U$ segue uma distribuição gama, cuja função densidade é

$$
f(u \mid \lambda, \phi)=\frac{(\phi / \lambda)^{\phi}}{\Gamma(\phi)} u^{\phi-1} \exp (-\phi u / \lambda), \quad u>0
$$

em que $\Gamma(x)=\int_{0}^{\infty} t^{x-1} \exp (-t) d t, x>0$, é a função gama. Isso implica que o valor esperado de $U$ é a concentração média $\lambda$ e que a correspondente variância é $\operatorname{Var}[U]=\lambda^{2} / \phi$. Diferentes valores de $\phi$ correspondem a diferentes maneiras segundo as quais os organismos se distribuem no tanque de lastro.

Pode-se supor que, condicionalmente a um valor específico $U=u$ para a concentração e ao parâmetro de forma $\phi$, o número de organismos $(X)$ em uma alíquota de água de lastro de $w \mathrm{~m}^{3}$ segue uma distribuição Poisson com média wu. Isso implica que (incondicionalmente) $X$ segue uma distribuição binomial negativa com média $w \lambda$ e parâmetro de forma $\phi$, i.e., com função de probabilidade

$$
\mathbb{P}[X=x \mid \phi, w, \lambda]=\frac{\Gamma(\phi+x)}{\Gamma(x+1) \Gamma(\phi)}\left(\frac{w \lambda}{w \lambda+\phi}\right)^{x}\left(\frac{\phi}{w \lambda+\phi}\right)^{\phi}, \quad \lambda, \phi>0
$$


para $x=0,1, \ldots$ Nesse contexto, $\mathbb{E}[X]=w \lambda$ e $\operatorname{Var}[X]=w \lambda+(w \lambda)^{2} / \phi$, indicando que esse modelo pode acomodar sobredispersão. O parâmetro $\phi$ é também conhecido na literatura como parâmetro de aglomeração, agrupamento ou heterogeneidade. No contexto da água de lastro, valores pequenos de $\phi$ correspondem a distribuições de organismos mais heterogêneas no tanque. Valores grandes de $\phi$, por outro lado, correspondem a distribuições mais homogêneas e nesse caso, a distribuição binomial negativa pode ser aproximada pela distribuição Poisson.

Suponha que $n$ alíquotas de $w \mathrm{~m}^{3}$ foram aleatoriamente coletadas de um tanque de lastro e que os correspondentes números de organismos, $X_{1}, \ldots, X_{n}$ seguem distribuições Poisson condicionalmente às suas respectivas médias, que seguem distribuição gama. Como consequência, $X_{i}, i=1, \ldots, n$ seguem uma distribuição binomial negativa com média $w \lambda$ e parâmetro de forma $\phi$ e $X=\sum_{i=1}^{n} X_{i}$ segue uma distribuição binomial negativa com média $n w \lambda$ e parâmetro de forma $n \phi$, para a qual, $\mathbb{E}[X]=n w \lambda=v \lambda$ e $\operatorname{Var}[X]=n w \lambda+(n w \lambda)^{2} / n \phi=v \lambda+(v \lambda)^{2} / n \phi$. Nesse caso, o valor crítico para a decisão sobre o cumprimento da norma D-2 $(c)$ e o número mínimo de alíquotas $(n)$ são determinados a partir de

$$
\beta_{1}=\mathbb{P}[X>c \mid \phi, w, \lambda=10] \quad \text { e } \quad \beta_{2}\left(\lambda_{A}\right)=\mathbb{P}\left[X \leq c \mid \phi, w, \lambda=\lambda_{A}\right],
$$

em que

$$
\mathbb{P}[X=x \mid \phi, w, \lambda]=\frac{\Gamma(n \phi+x)}{\Gamma(x+1) \Gamma(n \phi)}\left(\frac{w \lambda}{w \lambda+\phi}\right)^{x}\left(\frac{\phi}{w \lambda+\phi}\right)^{n \phi}, \quad \lambda, \phi>0,
$$

para $x=0,1, \ldots$, e $\lambda_{A}$ denota uma concentração média maior do que 10 pré-especificada. Para valores grandes de $\phi$ ou $n$, a distribuição de $X$ pode ser aproximada pela distribuição Poisson com média $v \lambda$. Mais detalhes podem ser obtidos em Costa et al. (2015). Um código em linguagem $\mathrm{R}$ (R Core Team, 2016) para os algoritmos correspondentes pode ser encontrado na seção "Supporting Information" de Costa et al. (2015) ou em www.ime.usp.br/ jmsinger/ballastwater.zip.

\subsection{Abordagem por meio de intervalos de confiança}

A abordagem por meio de teste de hipóteses é conveniente para tomada de decisão, mas não fornece a magnitude da estimativa da concentração média $\lambda$. Para incorporar essa característica, Costa et al. (2016) propõem uma solução baseada em intervalos de confiança.

Suponha que $n$ alíquotas com volume fixado $w$ foram aleatoriamente coletadas de um tanque e que os correspondentes números de organismos $X_{1}, \ldots, X_{n}$ seguem uma distribuição binomial negativa. Então, podemos usar o estimador de máxima verossimilhança $\widehat{\lambda}=(n w)^{-1} \sum_{i=1}^{n} X_{i}$, para 
estimar $\lambda$. Sob essa configuração, desejamos determinar o número mínimo de alíquotas tal que os limitantes superiores para o erro de estimação absoluto $(|\widehat{\lambda}-\lambda|)$ ou para o erro de estimação relativo $(|\widehat{\lambda}-\lambda| / \lambda)$ sejam $\epsilon_{a}>0$ e $\epsilon_{r} \in(0,1)$, respectivamente, com probabilidade mínima pré-especificada (nível de confiança) $1-\rho, \rho \in(0,1)$, i.e., determinar $n$ tal que, para todo $\lambda$,

$$
\mathbb{P}\left[|\widehat{\lambda}-\lambda|<\epsilon_{a}\right]>1-\rho \quad \text { ou } \quad \mathbb{P}\left[|\widehat{\lambda}-\lambda|<\epsilon_{r} \lambda\right]>1-\rho
$$

Chen $(2007,2011)$ e Chen \& Chen (2016) estabelecem um método para calcular o tamanho amostral mínimo para construir intervalos de confiança para a média de uma distribuição Poisson ou binomial com níveis de confiança fixados, controlando o erro de estimação absoluto, o erro de estimação relativo ou ambos. O único requisito é a especificação de limitantes inferior e superior para o parâmetro de interesse, i.e., a determinação de um intervalo $[a, b]$ ao qual o parâmetro pertence. Nessa configuração, Chen $(2007,2011)$ e Chen \& Chen (2016) mostram que o mínimo valor para $\mathbb{P}\left[|\widehat{\lambda}-\lambda|<\epsilon_{a}\right]$ ou para $\mathbb{P}\left[|\widehat{\lambda}-\lambda|<\epsilon_{r} \lambda\right]$, consideradas como funções de $\lambda$, pode ser determinado calculando essas probabilidades um número finito de vezes. Costa et al. (2016) estendem tais resultados para a distribuição binomial negativa e mostram como eles podem ser usados para construir algoritmos para calcular os tamanhos amostrais desejados.

Quando não há informação a priori sobre a concentração média, i.e., não há informação a priori de que $\lambda$ pertença a um intervalo $[a, b]$, Costa et al. (2016) propõem uma fórmula simples para calcular o número mínimo de alíquotas necessários para estimar $\lambda$, adaptando os resultados de Chen (2008) para a distribuição binomial negativa. Neste caso, entretanto, calcular o número mínimo de alíquotas requer que limitantes superiores para ambos os erros de estimação, absoluto e relativo, sejam especificados simultaneamente, i.e., o número mínimo de alíquotas $n$ deve ser tal que

$$
\mathbb{P}\left[|\widehat{\lambda}-\lambda|<\epsilon_{a} \text { ou }|\widehat{\lambda}-\lambda|<\epsilon_{r} \lambda\right]>1-\rho,
$$

em que $1-\rho, \rho \in(0,1)$, é o nível de confiança mínimo.

Nesta abordagem é suficiente fixar um nível de confiança, $1-\rho$, o volume da alíquota $w$, e os limitantes superiores para os erros de estimação absoluto e relativo. O número mínimo de alíquotas desejado é o menor inteiro $n$ maior do que

$$
\frac{\epsilon_{r}}{\epsilon_{a}} \times \frac{\log (2 / \rho)}{w\left(1+\epsilon_{r}\right) \log \left(1+\epsilon_{r}\right)-\left[\epsilon_{r} \phi / \epsilon_{a}+w\left(1+\epsilon_{r}\right)\right] \log \left[1+w \epsilon_{a} \epsilon_{r} /\left(\phi \epsilon_{r}+w \epsilon_{a}\right)\right]} .
$$

A diferença entre (1.5) e o correspondente resultado em Chen (2008), que prova o resultado para 
a distribuição Poisson com $w=1$, está no denominador, em que o termo $\epsilon_{r}$ ( $w \epsilon_{r}$ para o caso em que é considerada uma alíquota de volume $w$ ) da expressão (1) de Chen (2008) é substituído por

$$
\left[\frac{\epsilon_{r}}{\epsilon_{a}} \phi+w\left(1+\epsilon_{r}\right)\right] \log \left(1+\frac{w \epsilon_{a} \epsilon_{r}}{\phi \epsilon_{r}+w \epsilon_{a}}\right)
$$

Uma vez que esse termo é maior do que $w \epsilon_{r}$, claramente o número de alíquotas obtido sob a distribuição binomial negativa é maior do que aquele calculado sob a distribuição Poisson com os mesmos valores para $w, \epsilon_{a}, \epsilon_{r}$ e $\rho$. Note também que somente o segundo termo do denominador de (1.5) depende de $\phi$. O limite de (1.6) quando $\phi$ tende ao infinito, i.e., quando a distribuição da concentração torna-se mais homogênea, é $w \epsilon_{r}$, o que sugere que para grandes valores do parâmetro de forma $\phi$, o número de alíquotas desejado pode ser obtido por meio da distribuição Poisson (Costa et al., 2016).

Usualmente, a decisão com respeito ao cumprimento da norma D-2 deve ser tomada depois de a amostra inteira ser coletada. Isto pode ser um problema quando a concentração média $\lambda$ é grande, uma vez que o não cumprimento da norma possivelmente será detectado somente depois que todo o processo de amostragem seja completado. Para contornar esse problema, Costa et al. (2016) sugerem um procedimento sequencial segundo o qual o não cumprimento dessa norma poderia ser avaliado à medida que a água de lastro é amostrada. Suponha que $n$ alíquotas sejam necessárias para garantir que o erro de estimação cumpra os limites especificados. Considere uma decisão baseada nas primeiras $n / K$ alíquotas em que $K$ é um inteiro positivo fixo. Dando ao proprietário do navio o benefício da dúvida, i.e., supondo que nenhum organismo seria detectado nas $n-n / K$ alíquotas restantes, usamos essas "pseudo-observações" juntamente com aquelas obtidas nas primeiras $n / K$ alíquotas para estimar a concentração média $\lambda$. Se o limite inferior do intervalo de confiança correspondente para a concentração média $\lambda$ for maior do que aquele especificado pela norma D-2, então declaramos que não há cumprimento. Em caso contrário, desprezamos as pseudo-observações, tomamos $n / K$ alíquotas adicionais e supomos que nenhum organismo seria detectado nas restantes $n-2 n / K$ alíquotas e repetimos o processo de decisão. O procedimento é repetido até tomarmos uma decisão ou completarmos $n$ observações.

A determinação do tamanho amostral é altamente dependente da heterogeneidade da distribuição dos organismos. Embora a abordagem apresentada neste capítulo forneça resultados úteis, eles são essencialmente dependentes da especificação da distribuição dos organismos no tanque de lastro, para a qual em geral, não há muitas informações disponíveis. Isso sugere a adoção de modelos mais flexíveis que eventualmente possam ser baseados em conhecimentos adquiridos ao longo do tempo. 
Uma alternativa que incorpora essa flexibilidade pode ser conseguida por meio da utilização de métodos bayesianos não-paramétricos baseados no processo Dirichlet (Ferguson, 1973). Os métodos bayesianos também permitem que a distribuição a posteriori seja atualizada durante o processo de deslastre e o estabelecimento de regras de decisão para identificar se a norma D-2 é cumprida.

O objetivo desta tese é estabelecer metodologias que permitam obter o tamanho amostral mínimo necessário para estimar a concentração média de organismos no tanque de lastro por meio de inferência intervalar.

Esta tese é organizada como segue. No Capítulo 2 são apresentados os modelos bayesianos, nomeadamente (1) o modelo Poisson com distribuição a priori gama para a concentração de organismos $\lambda$; (2) o modelo binomial negativo com distribuição a priori Pearson Tipo VI para $\lambda$ (embora esses modelos já tenham sido utilizados na abordagem frequentista, iremos considerá-los sob a ótica bayesiana); (3) o modelo Poisson com distribuição a priori não-paramétrica para $\lambda$. Nesse caso, consideramos uma distribuição Poisson para as contagens observadas, supondo que as concentrações subjacentes seguem uma distribuição proveniente de um processo Dirichlet. Esse modelo acrescenta mais flexibilidade à especificação do conhecimento a priori sobre a concentração média de organismos e pode englobar mais situações práticas. No Capítulo 3 abordamos critérios para calcular o tamanho amostral a partir dos modelos bayesianos apresentados no Capítulo 2. Por fim, no Capítulo 4 apresentamos uma discussão dos resultados. 


\section{Capítulo 2}

\section{Modelos bayesianos}

\subsection{Modelo Poisson com distribuição a priori gama}

Dada a concentração média $\lambda$, seja $X$ o número de organismos em uma alíquota de volume $w$; nessa alíquota, esperamos encontrar $w \lambda$ organismos, ou $\mathbb{E}[X \mid \lambda]=w \lambda$. Suponha que, dado $\lambda, X$ segue uma distribuição Poisson com média $w \lambda$, i.e., que os organismos se distribuem no tanque de forma homogênea.

Para especificar o modelo bayesiano precisamos atribuir uma distribuição de probabilidade para o parâmetro de interesse $(\lambda)$ que irá refletir o nosso conhecimento a priori. Uma possível escolha é uma distribuição de probabilidade a priori para $\lambda$ que seja a conjugada natural da distribuição Poisson; neste caso será a distribuição gama, de forma que

$$
F(d \lambda) \propto \lambda^{\theta_{0}-1} \exp \left(-\theta_{0} \lambda / \lambda_{0}\right)
$$

em que $\lambda_{0}$ e $\theta_{0}$ são constantes fixas positivas e conhecidas (hiperparâmetros); utilizamos a notação $\lambda \sim G\left(\theta_{0}, \theta_{0} / \lambda_{0}\right)$. Neste caso, temos $\mathbb{E}[\lambda]=\lambda_{0}$ e $\operatorname{Var}[\lambda]=\lambda_{0}^{2} / \theta_{0}$. O parâmetro $\lambda_{0}$ representa uma estimativa a priori para a concentração média $\lambda$ e $\theta_{0}$ controla a variabilidade em torno de $\lambda_{0}$ de modo que quanto maior (menor) for $\theta_{0}$, menor (maior) será a incerteza a priori sobre $\lambda$.

A distribuição gama, além de ser a conjugada natural da distribuição Poisson, possui flexibilidade na especificação do conhecimento a priori, podendo assumir várias formas, desde aquelas com altas probabilidade associadas a concentrações médias próximas de zero $\left(\lambda_{0}=3, \theta_{0}=1\right)$, altas probabilidades associadas a concentrações médias em torno de 5 ou $20\left(\lambda_{0}=5, \theta_{0}=20\right.$ e $\lambda_{0}=20$, $\left.\theta_{0}=350\right)$, até aquelas que exprimem pouco conhecimento a priori, com probabilidades distribuídas ao longo de valores pequenos e grandes $\left(\lambda_{0}=18, \theta_{0}=3\right)$ para a concentração média (Figura 2.1). 


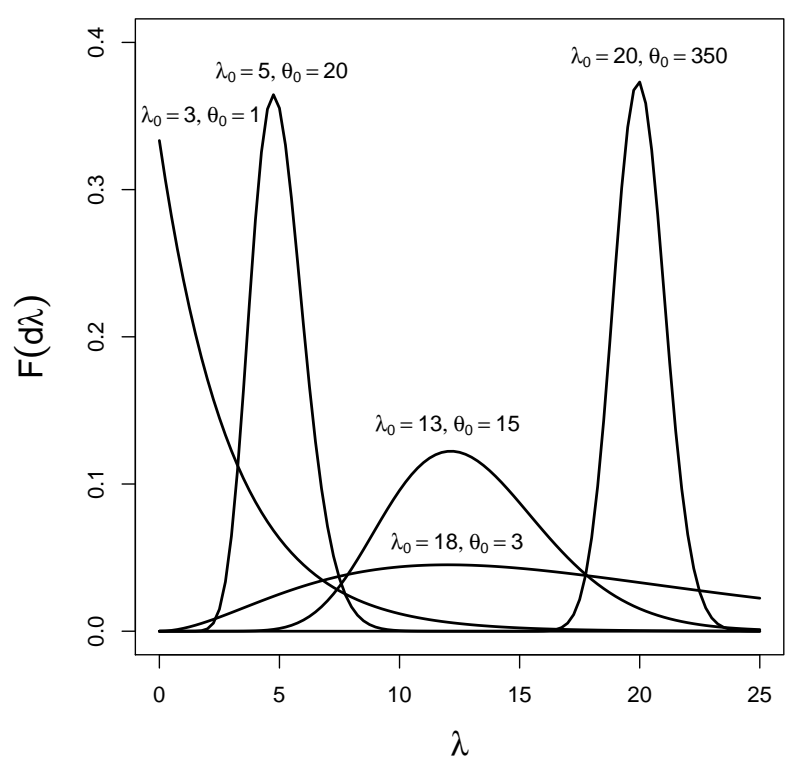

Figura 2.1: Funções densidade da distribuição $G\left(\theta_{0}, \theta_{0} / \lambda_{0}\right)$.

Considere uma amostra aleatória de tamanho $n$ de $X$ dado $\lambda$ e uma distribuição a priori gama para $\lambda$. Podemos então escrever o modelo da seguinte forma hierárquica:

$$
\begin{aligned}
& X_{i} \mid \lambda \stackrel{\mathrm{iid}}{\sim} \operatorname{Poi}(w \lambda), \quad i=1,2, \ldots, n \\
& \lambda \sim F:=G\left(\theta_{0}, \theta_{0} / \lambda_{0}\right) .
\end{aligned}
$$

Neste contexto, a distribuição a posteriori de $\lambda$ também será gama, com parâmetros $\theta_{0}+s_{n}$ e $n w+\theta_{0} / \lambda_{0}$, em que $s_{n}=\sum_{i=1}^{n} x_{i}$, i.e., $\lambda \mid \boldsymbol{x}_{n} \sim G\left(\theta_{0}+s_{n}, n w+\theta_{0} / \lambda_{0}\right)$, em que $\boldsymbol{x}_{n}=\left(x_{1}, \ldots, x_{n}\right)$. Detalhes podem ser encontrados no Apêndice A.

\subsection{Modelo binomial negativo com distribuição a priori Pearson Tipo VI}

Em contrapartida à suposição de homogeneidade dos organismos no tanque considerada na distribuição Poisson, considere uma situação mais realista em que os organismos se distribuem de forma heterogênea. Nesse sentido, a $i$-ésima alíquota terá uma concentração $\lambda_{i}$ de organismos e um número de organismos correspondente, que denotaremos por $X_{i}, i=1, \ldots, n$. Assim, na $i$-ésima alíquota esperamos encontrar $w \lambda_{i}$ organismos, ou $\mathbb{E}\left[X_{i} \mid \lambda_{i}\right]=w \lambda_{i}$. Para $i=1, \ldots, n$, suponha que, dado $\lambda_{i}, X_{i}$ segue uma distribuição Poisson com média $w \lambda_{i}$ e que dada uma concentração média $\lambda$ no tanque, $\lambda_{i} \sim G(\phi, \phi / \lambda)$, i.e., $\mathbb{E}\left[\lambda_{i} \mid \lambda\right]=\lambda$ e $\operatorname{Var}\left[\lambda_{i} \mid \lambda\right]=\lambda^{2} / \phi$. Logo, dado $\lambda$ e $\phi, X_{i}$ segue uma 
distribuição binomial negativa com $\mathbb{E}\left[X_{i} \mid \lambda, \phi\right]=w \lambda$ e $\operatorname{Var}\left[X_{i} \mid \lambda, \phi\right]=w \lambda+(w \lambda)^{2} / \phi$, em que $\phi$ é um parâmetro de forma (ou aglomeração) suposto conhecido; denotamos por $X_{i} \mid \lambda, \phi \sim B N(w \lambda, \phi)$.

Uma possível distribuição de probabilidade a priori $F$ para $\lambda$ e que é a conjugada natural da distribuição binomial negativa é a distribuição Pearson Tipo VI, também conhecida como distribuição beta-linha (Johnson et al., 1994a,b), i.e.,

$$
F(d \lambda) \propto\left(\frac{w}{\phi} \lambda\right)^{\theta_{0}-1}\left(1+\frac{w}{\phi} \lambda\right)^{-\theta_{0}-\left(\theta_{0} / \lambda_{0}+1\right)}
$$

com parâmetro de localização 0 , parâmetro de escala $\phi / w$ e parâmetros de forma $\theta_{0}$ e $\theta_{0} / \lambda_{0}+1$, em que $\lambda_{0}$ e $\theta_{0}$ são constantes fixas positivas e conhecidas (hiperparâmetros). Utilizamos a notação $\lambda \sim P V I\left(0, \phi / w, \theta_{0}, \theta_{0} / \lambda_{0}+1\right)$. Neste caso, temos $\mathbb{E}[\lambda]=(\phi / w) \lambda_{0}$ e $\operatorname{Var}[\lambda]=\left(\lambda_{0}^{2} / \theta_{0}\right)\left[\phi^{2}\left(\lambda_{0}+\right.\right.$ $\left.1) /\left(w^{2}\left(1-\lambda_{0} / \theta_{0}\right)\right)\right]$, para $\lambda_{0}<\theta_{0}$.

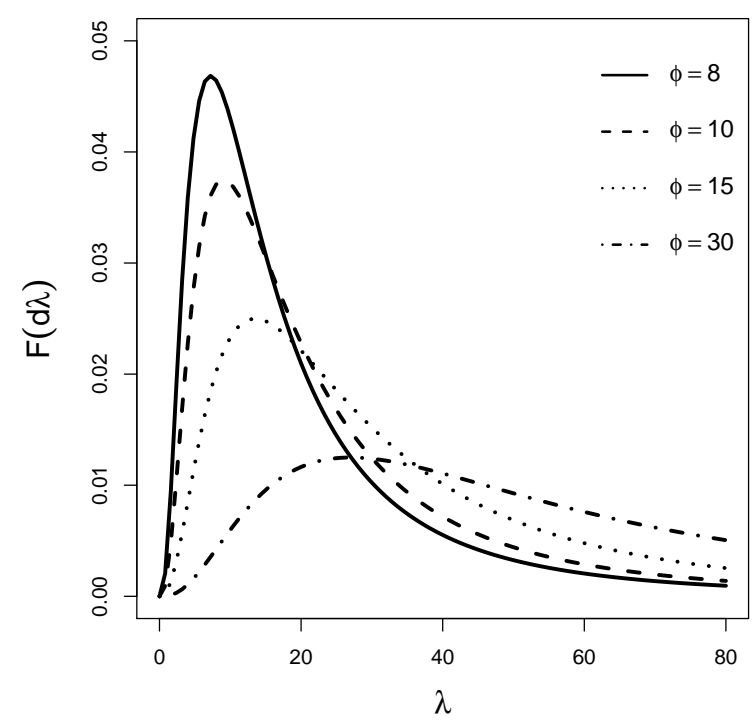

Figura 2.2: Funções densidade da distribuição $P V I\left(0, \phi / w, \theta_{0}, \theta_{0} / \lambda_{0}+1\right)$ com $w=1, \lambda_{0}=3$ e $\theta_{0}=4$.

No modelo Poisson com distribuição a priori gama, temos $\mathbb{E}[X]=\mathbb{E}[\mathbb{E}[X \mid \lambda]]=\mathbb{E}[w \lambda]=w \lambda_{0}$, i.e., a quantidade de organismos esperada ao se coletar uma alíquota depende exclusivamente do hiperparâmetro $\lambda_{0}$. Isso faz sentido dado que estamos supondo a homogeneidade da concentração e independentemente do local do tanque de lastro em que coletássemos uma amostra, esperaríamos encontrar a mesma quantidade de organismos. Por outro lado, se considerarmos o modelo binomial negativo com distribuição a priori Pearson Tipo VI, temos $\mathbb{E}[X]=\phi \lambda_{0}$; logo, a quantidade esperada de organismos em uma alíquota depende também do parâmetro $\phi$ que controla a heterogeneidade dos organismos dentro do tanque. Note também que $\phi$ é um parâmetro de escala da distribuição a priori e quanto maior o seu valor, mais dispersa é a distribuição (fixando-se os outros parâmetros) 

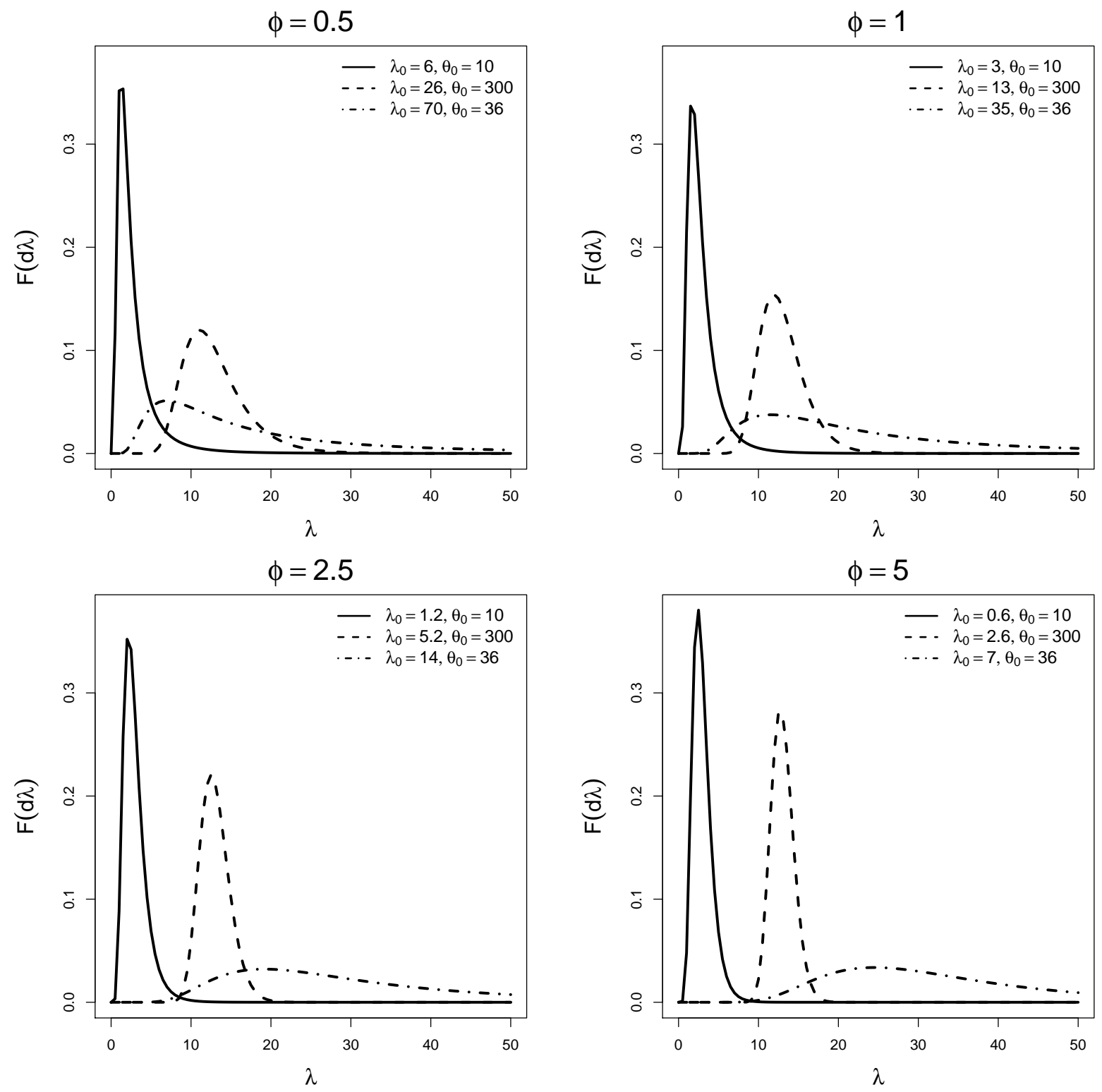

Figura 2.3: Funções densidade da distribuição $P V I\left(0, \phi / w, \theta_{0}, \theta_{0} / \lambda_{0}+1\right)$ com $w=1$.

indicando conhecimento a priori vago sobre o parâmetro de interesse. Felizmente, podemos ajustar os outros parâmetros de maneira que a distribuição a priori possa representar o caso em que há grande probabilidade associada a um determinado intervalo mesmo quando o valor de $\phi$ aumenta (Figura 2.3). Quando $\lambda_{0}$ e $\theta_{0}$ são fixados e $\phi$ aumenta, temos cada vez mais distribuições representando situações com maior variabilidade (Figura 2.2). Neste caso, o conhecimento do valor de $\phi$ é essencial para a simplificação da inferência a posteriori de forma que não seria possível haver conjugação dos modelos se $\phi$ fosse desconhecido. Se considerássemos $\phi$ desconhecido, teríamos que atribuir uma distribuição a priori para $(\lambda, \phi)$ e provavelmente utilizar métodos MCMC (Markov chain Monte Carlo) para realizar inferências a posteriori.

Considere uma amostra aleatória de tamanho $n$ de $X$, dados $\lambda$ e $\phi$, e uma distribuição a priori 
Pearson Tipo VI para $\lambda$. Podemos então escrever o modelo da seguinte forma hierárquica:

$$
\begin{aligned}
& X_{i} \mid \lambda, \phi \stackrel{\mathrm{iid}}{\sim} B N(w \lambda, \phi), \quad i=1,2, \ldots, n \\
& \lambda \sim F:=\operatorname{PVI}\left(0, \phi / w, \theta_{0}, \theta_{0} / \lambda_{0}+1\right) .
\end{aligned}
$$

Neste contexto, a distribuição a posteriori de $\lambda$ também será Pearson Tipo VI, com os mesmos parâmetros de localização e escala da distribuição a priori e parâmetros de forma $\theta_{0}+s_{n}$ e $\theta_{0} / \lambda_{0}+n \phi+1$, i.e., $\lambda \mid \boldsymbol{x}_{n} \sim P V I\left(0, \phi / w, \theta_{0}+s_{n}, \theta_{0} / \lambda_{0}+n \phi+1\right)$. Detalhes são apresentados no Apêndice B.

\subsection{Modelo Poisson com distribuição a priori não-paramétrica}

Supor o modelo (2.3)-(2.4) para a concentração de organismos em um tanque de lastro implica que a distribuição dessa concentração é regida por uma distribuição unimodal de probabilidade, o que pode não ser apropriado dado que podemos ter o caso onde duas ou mais regiões do tanque possuem alta concentração de organismos. Uma alternativa é considerar um processo Dirichlet para a construção dessa distribuição (Antoniak, 1974), como fizeram Bush \& MacEachern (1996) que utilizam o processo Dirichlet para modelar o efeito de blocos no delineamento em blocos aleatorizados, Escobar (1994) que usa o processo Dirichlet na estimação de médias de distribuições normais e Escobar \& West (1995) que usam misturas de processos Dirichlet para a estimação de densidades, entre outros exemplos de aplicação.

Seja $\lambda_{i}, i=1, \ldots, n$, a concentração de organismos associada à $i$-ésima alíquota de uma amostra aleatória e suponha que $\lambda_{i}$ segue uma distribuição $F$. Na abordagem paramétrica, supomos uma forma conhecida para $F$, indexada por um ou mais parâmetros. Ao invés de especificar a forma dessa distribuição $F$, podemos estabelecer um conjunto de distribuições possíveis no qual $F$ pode transitar, o que permite maior flexibilidade na modelagem e robustez contra a má especificação. Uma maneira de evitar a especificação de uma forma paramétrica para $F$ é empregar uma medida de probabilidade aleatória (MPR), que são distribuições de probabilidade sobre o espaço de medidas de probabilidade (em $\mathbb{R}_{+}$) e um exemplo de uma MPR é o processo Dirichlet. Ferguson (1973) propôs o processo Dirichlet como uma possível solução para o problema da especificação de distribuições a priori sob uma abordagem bayesiana não-paramétrica em que o espaço paramétrico é um conjunto de distribuições de probabilidade sobre um dado espaço amostral.

Admitamos que $F$ segue um processo Dirichlet com parâmetro $\alpha\left(\cdot ; \alpha^{*}, F_{0}\right)=\alpha^{*} F_{0}(\cdot)$, 
simbolicamente $F \sim D P\left[\alpha\left(\cdot ; \alpha^{*}, F_{0}\right)\right]$. Sob essa parametrização, temos $\mathbb{E}[F(A)]=F_{0}(A)$ e $\operatorname{Var}[F(A)]=F_{0}(A)\left[1-F_{0}(A)\right] /\left(\alpha^{*}+1\right)$, em que $A$ pertence à $\sigma$-álgebra de $\Lambda$ (espaço paramétrico de $\lambda), F_{0}$ é chamado de distribuição base e $\alpha^{*}$ um parâmetro de escala. Note que o termo "nãoparamétrica" aqui se refere ao fato de a estrutura da distribuição a priori do modelo não ser fixa quando consideramos um processo Dirichlet. Para mais detalhes, vide Apêndice C.

As propriedades e a facilidade com que a distribuição a posteriori é obtida evidenciam o motivo pelo qual o processo Dirichlet é tão atrativo quando analisado sob uma perspectiva bayesiana. Note que em nosso caso, o processo Dirichlet é uma distribuição a priori atribuída à distribuição das concentrações médias associadas às observações poissonianas condicionantes. Nesse sentido, podemos escrever o modelo hierarquicamente como

$$
\begin{aligned}
& X_{i} \mid \lambda_{i} \stackrel{\text { ind }}{\sim} \operatorname{Poi}\left(w \lambda_{i}\right), \quad i=1,2, \ldots, n \\
& \lambda_{i} \mid F \stackrel{\mathrm{iid}}{\sim} F, \quad i=1,2, \ldots, n \\
& F \sim D P\left[\alpha\left(\cdot ; \alpha^{*}, F_{0}\right)\right] .
\end{aligned}
$$

Esta especificação define um modelo de mistura por processo Dirichlet, tal como é indicado em (C.6)-(C.8) no Apêndice C. A distribuição a posteriori da medida $F$ é dada por

$$
F\left(A \mid \boldsymbol{x}_{n}\right)=\int_{\Lambda^{n}} F\left(A \mid \boldsymbol{\lambda}_{n}\right) \nu\left(d \boldsymbol{\lambda}_{n} \mid \boldsymbol{x}_{n}\right)
$$

em que $\boldsymbol{x}_{n}=\left(x_{1}, \ldots, x_{n}\right), \boldsymbol{\lambda}_{n}=\left(\lambda_{1}, \ldots, \lambda_{n}\right), F\left(A \mid \boldsymbol{\lambda}_{n}\right)$ é um processo Dirichlet com parâmetro $\alpha\left(A ; \alpha^{*}, F_{0}, \lambda_{n}\right)=\alpha^{*} F_{0}(A)+\sum_{i=1}^{n} \delta_{\lambda_{i}}(A), \operatorname{com} \delta_{\lambda_{i}}(A)=1$, se $\lambda_{i} \in A$, e $\delta_{\lambda_{i}}(A)=0$, em caso contrário,

$$
\nu\left(d \boldsymbol{\lambda}_{n} \mid \boldsymbol{x}_{n}\right) \propto \prod_{i=1}^{n} g\left(x_{i} \mid \lambda_{i}\right)\left[\alpha^{*} F_{0}\left(d \lambda_{i}\right)+\sum_{j=1}^{i-1} \delta_{\lambda_{i}}\left(d \lambda_{j}\right)\right],
$$

e $g\left(x_{i} \mid \lambda_{i}\right)$ representando a função de probabilidade da distribuição $\operatorname{Poi}\left(w \lambda_{i}\right)$. A distribuição $F\left(A \mid \boldsymbol{x}_{n}\right)$ é assim uma mistura de processos Dirichlet $\operatorname{com} \nu\left(d \boldsymbol{\lambda}_{n} \mid \boldsymbol{x}_{n}\right)$ como distribuição misturadora (vide Antoniak, 1974).

Na prática podemos considerar $A$ como sendo um intervalo semi-aberto $(-\infty, t]$ de maneira a obtermos uma função distribuição acumulada. Note que como $F\left(A \mid \boldsymbol{x}_{n}\right)$ é uma medida de probabilidade aleatória, utilizaremos o seu valor esperado, $\mathbb{E}\left[F\left(A \mid \boldsymbol{x}_{n}\right)\right]$, para fazer inferências sobre o parâmetro de interesse. Detalhes estão apresentados no Apêndice C. 


\section{Capítulo 3}

\section{Determinação do tamanho amostral}

\section{sob o enfoque bayesiano}

A determinação do tamanho amostral envolve basicamente dois componentes: um modelo probabilístico e um critério sob o qual tamanho amostral $n$ será determinado. No contexto do problema da estimação da concentração média de organismos em água de lastro, Costa et al. (2015) consideram as distribuições Poisson e binomial negativa. Como critério para a obtenção do tamanho amostral $n$, esses autores fixam as probabilidades dos erros do Tipo I e do Tipo II para testar a hipótese de que a concentração média de organismos é menor do que ou igual a 10. Por outro lado, Costa et al. (2016) também consideram a distribuição binomial negativa e como critério para determinação do tamanho da amostra escolhem a minimização da probabilidade de que a diferença entre a concentração média e sua estimativa seja menor que um valor pré-fixado. Ambas as abordagens de Costa et al. $(2015,2016)$ são frequentistas.

$\mathrm{Na}$ abordagem bayesiana, os primeiros critérios para determinação do tamanho amostral que consideramos são os da cobertura média e do comprimento médio de intervalos de credibilidade (ACC e ALC, respectivamente, nas siglas em inglês). Esses critérios são amplamente utilizados na literatura. Adcock $(1987,1988)$ utiliza o ACC (ainda que não use essa nomenclatura) para determinar tamanhos amostrais nos problemas de estimação do vetor de probabilidades, sob o modelo multinomial com distribuição a priori Dirichlet e da média e da variância sob o modelo normal, com distribuições a priori normal e qui-quadrado para os casos em que a variância é conhecida e desconhecida, respectivamente. Já Joseph et al. (1995) e Joseph et al. (1997) utilizam os critérios ACC e ALC, entre outros critérios bayesianos nos problemas de estimação de uma proporção e da diferença entre duas proporções sob o modelo binomial com distribuição a priori 
beta. Em problemas envolvendo a estimação da média sob o modelo normal, da proporção sob o modelo binomial, do parâmetro do modelo Poisson e do parâmetro referente à inclinação da reta em regressão linear simples, Adcock (1997) discute os critérios ACC, ALC e outros critérios bayesianos. Wang \& Gelfand (2002) usam os mesmos critérios bayesianos discutidos em Adcock (1997) para determinar o tamanho amostral em problemas envolvendo a estimação de um parâmetro de uma distribuição da família exponencial, a estimação em modelos de sobrevivência com distribuição Weibull e a estimação em regressão logística entre outros. M'Lan et al. (2006) utilizam os critérios ACC e ALC em modelos para estudos caso-controle; Stamey et al. (2006) também consideram esses critérios para a estimação do parâmetro do modelo Poisson, bem como para a estimação da diferença e da razão entre os parâmetros de dois modelos Poisson, e Nassar et al. $(2010,2011)$ os utilizam na estimação da probabilidade do modelo geométrico com distribuição a priori beta e no modelo Laplace com distribuição a priori normal. Trabalhos que seguem a mesma abordagem bayesiana são Joseph \& Bélisle (1997), Joseph \& Wolfson (1997), Rahme et al. (2000), De Santis (2007), M'Lan et al. (2008), entre outros.

Ainda sob a abordagem bayesiana, outro critério utilizado neste trabalho é a minimização do custo total (MCT) definido como a soma do risco de Bayes e de uma função custo. Etzioni \& Kadane (1993) seguem esse critério e utilizam funções perda quadrática e logarítmica sob o modelo normal. Sahu \& Smith (2006) consideram uma função perda no problema de teste de hipóteses para um parâmetro do modelo normal. Islam $(2011)$ e Islam \& Pettit $(2012,2014)$ consideram funções perda quadrática, linex, linex limitada, entre outras, no problema de estimação pontual da média e da variância sob o modelo normal com distribuição a priori normal, sob os modelos exponencial e Poisson ambos com distribuição a priori gama na estimação pontual dos seus respectivos parâmetros, entre outros. Trabalhos que também seguem o critério MCT ou um critério que requer a especificação de uma função perda para o problema são apresentados em Pham-Gia \& Turkkan (1992), Bernardo (1997), Lindley (1997), Brutti et al. (2008, 2009), Parmigiani \& Inoue (2009), Brutti et al. (2014), De Santis \& Gubbiotti (2016), entre outros.

\subsection{Critérios para determinar o tamanho amostral}

\subsubsection{Critério da cobertura média (CCobM)}

O primeiro critério que consideramos para determinar o tamanho amostral para estimar $\lambda$ com uma precisão pré-especificada é o critério da cobertura média de regiões de credibilidade. Dada uma amostra $\boldsymbol{x}_{n}$, podemos determinar o tamanho amostral mínimo $n$ para que uma região de 
credibilidade $R\left(\boldsymbol{x}_{n}\right)$ com uma amplitude pré-especificada tenha probabilidade a posteriori no mínimo igual a $1-\rho$, i.e.,

$$
\int_{R\left(\boldsymbol{x}_{n}\right)} F\left(d \lambda \mid \boldsymbol{x}_{n}\right) \geq 1-\rho
$$

em que $R\left(\boldsymbol{x}_{n}\right)$ é um subconjunto (em nosso caso, um intervalo ou união de intervalos disjuntos para distribuições multimodais) do espaço paramétrico. Uma vez que a determinação do tamanho amostral $n$ deve preceder a amostragem, precisamos considerar todos os possíveis resultados para $\boldsymbol{x}_{n}$. Uma alternativa é ponderar cada resultado possível por sua respectiva probabilidade; nesse caso, o critério resume-se em obter o menor valor de $n$ tal que

$$
\int_{\mathcal{X}^{n}}\left[\int_{R\left(\boldsymbol{x}_{n}\right)} F\left(d \lambda \mid \boldsymbol{x}_{n}\right)\right] g\left(\boldsymbol{x}_{n}\right) d \boldsymbol{x}_{n} \geq 1-\rho,
$$

em que $\mathcal{X}^{n}$ é o espaço amostral de $\boldsymbol{x}_{n}$ e $g\left(\boldsymbol{x}_{n}\right)$ é a função de probabilidade (ou densidade) marginal dos dados. Note que outras medidas para resumir todos resultados possíveis para $\boldsymbol{x}_{n}$ além da média podem ser utilizadas, como indicado em M'Lan et al. (2008). Por exemplo, pode-se considerar a mediana em relação à $\boldsymbol{x}_{n}$, i.e.,

$$
\underset{\boldsymbol{x}_{n} \in \mathcal{X}_{n}}{\operatorname{med}}\left[\int_{R\left(\boldsymbol{x}_{n}\right)} F\left(d \lambda \mid \boldsymbol{x}_{n}\right)\right] \geq 1-\rho .
$$

Considerando os modelos (2.1)-(2.2) e (2.3)-(2.4), a moda da distribuição a posteriori será única e a região de credibilidade consistirá de um único intervalo, neste caso tomaremos $R\left(\boldsymbol{x}_{n}\right)=[a, b]$ como o intervalo HPD (highest posterior density). Com essa finalidade fixamos o tamanho $\ell>0$ do intervalo HPD para $\lambda$, a probabilidade de cobertura bayesiana mínima, $1-\rho$, e determinamos o tamanho amostral mínimo além dos limitantes $a$ e $b=a+\ell$ tais que

$$
\int_{\mathcal{X}^{n}}\left[\int_{a}^{b} F\left(d \lambda \mid \boldsymbol{x}_{n}\right)\right] g\left(\boldsymbol{x}_{n}\right) d \boldsymbol{x}_{n} \geq 1-\rho .
$$

A integral interna de (3.1) pode ser facilmente calculada computacionalmente dados $a, b$ e os parâmetros da distribuição a posteriori; já a integral externa precisa ser estimada por meio de simulações de Monte Carlo. Segue uma descrição resumida de como estimar (3.1) para um dado $n$ : simule $\boldsymbol{x}_{n}$, calcule os limitantes $a$ e $b$, em seguida calcule a integral interna de (3.1), repita o procedimento anterior uma quantidade considerável de vezes (e.g., 1000 vezes) obtendo 1000 valores para a integral interna. A média desses 1000 valores será a estimativa desejada. Fixa-se um valor inicial para $n$ que vai sendo incrementado até que o critério (3.1) seja satisfeito. No modelo (2.1)-(2.2) 
a distribuição a posteriori depende de $\boldsymbol{x}_{n}$ através de $s_{n}$ e dado que neste caso $S_{n}=\sum_{i=1}^{n} X_{i}$ segue uma distribuição binomial negativa, então no início do processo descrito anteriormente ao invés de simular $\boldsymbol{x}_{n}$ simulamos $s_{n}$ diretamente. Algoritmos mais detalhados para implementar o cálculo do tamanho amostral sob os modelos (2.1)-(2.2) e (2.3)-(2.4) encontram-se em A.4.1 e B.3.1 dos Apêndices A e B, respectivamente.

Considerando o modelo (2.5)-(2.7), a distribuição a posteriori poderá ser multimodal; consequentemente, a região HPD poderá consistir da união de intervalos disjuntos. Fixamos o tamanho $\ell$ da região HPD para $\lambda$, a probabilidade de cobertura bayesiana mínima $1-\rho$ e determinamos o tamanho amostral mínimo, além da região $\mathrm{HPD}, R\left(\boldsymbol{x}_{n}\right)$, tais que

$$
\int_{\mathcal{X}^{n}} \mathbb{E}\left[F\left(R\left(\boldsymbol{x}_{n}\right) \mid \boldsymbol{x}_{n}\right)\right] g\left(\boldsymbol{x}_{n}\right) d \boldsymbol{x}_{n} \geq 1-\rho .
$$

Neste caso, tanto o valor esperado quanto a integral de (3.2) devem ser estimadas por meio de simulações de Monte Carlo. De forma resumida, podemos estimar (3.2) para um dado $n$ da seguinte forma: simule $\boldsymbol{x}_{n}$, obtenha a região $\operatorname{HPD} R\left(\boldsymbol{x}_{n}\right)$ e em seguida calcule uma estimativa de $\mathbb{E}\left[F\left(R\left(\boldsymbol{x}_{n}\right) \mid \boldsymbol{x}_{n}\right)\right]$, repita o procedimento anterior (e.g., 1000 vezes), obtendo assim 1000 estimativas para o valor esperado. A média dessas 1000 estimativas é uma estimativa para (3.2). Fixa-se um valor inicial para $n$ que vai sendo incrementado até que o critério (3.2) seja satisfeito. Algoritmos mais detalhados para obter a região HPD $R\left(\boldsymbol{x}_{n}\right)$ e o tamanho amostral encontram-se em C.3.3, C.3.4 e C.3.5 do Apêndice C.

\subsubsection{Critério do comprimento médio (CCompM)}

Outra alternativa para determinar o tamanho amostral é usar um critério baseado no comprimento médio de regiões de credibilidade (a posteriori). Primeiro fixamos a probabilidade de cobertura bayesiana mínima $1-\rho$ e obtemos o respectivo tamanho da região de credibilidade a posteriori denotado por $\ell^{\prime}\left(\boldsymbol{x}_{n}, n\right)$. Então, determinamos o tamanho amostral mínimo $n$ tal que

$$
\int_{\mathcal{X}^{n}} \ell^{\prime}\left(\boldsymbol{x}_{n}, n\right) g\left(\boldsymbol{x}_{n}\right) d \boldsymbol{x}_{n} \leq \ell_{\max }
$$

em que $\ell_{\max }$ é o tamanho máximo admissível fixado para a região de credibilidade a posteriori.

Considerando os modelos (2.1)-(2.2) e (2.3)-(2.4), a região de credibilidade consistirá de um único intervalo. Denotando $a$ e $b$ os limitantes inferior e superior do intervalo HPD de probabilidade $1-\rho$ da distribuição a posteriori, o respectivo tamanho do intervalo é $\ell^{\prime}\left(\boldsymbol{x}_{n}, n\right)=b-a$. O tamanho 
$\ell^{\prime}\left(\boldsymbol{x}_{n}, n\right)$ pode ser obtido calculando-se os limitantes $a$ e $b$ por meio de métodos numéricos; e a integral relacionada ao critério pode ser estimada por meio de simulações de Monte Carlo. Uma descrição resumida para obter uma estimativa da integral em (3.3) para um dado $n$ é: simule $\boldsymbol{x}_{n}$, obtenha $a$ e $b$, e em seguida calcule $\ell^{\prime}\left(\boldsymbol{x}_{n}, n\right)=b-a$, repita o procedimento anterior (e.g., 1000 vezes), obtendo assim 1000 valores para $\ell^{\prime}\left(\boldsymbol{x}_{n}, n\right)$. A média desses 1000 valores é uma estimativa para a integral em (3.3). Fixa-se um valor inicial para $n$ que vai sendo incrementado até que o critério (3.3) seja satisfeito.

Por outro lado, se considerarmos o modelo (2.5)-(2.7), a região HPD da distribuição a posteriori $\mathbb{E}\left[F\left(\cdot \mid \boldsymbol{x}_{n}\right)\right]$ poderá consistir da união de intervalos disjuntos do espaço paramétrico e neste caso consideraremos como o tamanho da região HPD a soma dos comprimentos dos respectivos intervalos disjuntos. Algoritmos mais detalhados para implementar o cálculo do tamanho amostral encontram-se em A.4.2, B.3.2 e C.3.6 dos Apêndices A, B e C, respectivamente.

Com base em M'Lan et al. (2008), que usaram o modelo binomial com distribuição a priori beta, obtemos o seguinte resultado que nos permite calcular o tamanho amostral usando o CCompM sob o modelo (2.1)-(2.2) sem a necessidade de utilizar simulações.

Teorema 3.1. Considere o modelo (2.1)-(2.2) e o critério do comprimento médio (3.3); então o número necessário de observações para que o comprimento médio da região de credibilidade a posteriori seja menor que $\ell_{\max } e^{\prime}$

$$
n \geq \frac{\theta_{0}}{w \lambda_{0}}\left\{\left[\frac{\lambda_{0}}{\theta_{0}} \frac{2 z_{\rho / 2}}{\ell_{\max }} \frac{\Gamma\left(\theta_{0}+1 / 2\right)}{\Gamma\left(\theta_{0}\right)}\right]^{2}-1\right\}
$$

em que $z_{\rho / 2}$ é o quantil de probabilidade $1-\rho / 2$ da distribuição normal padrão.

Demonstração. Vide Apêndice A.

\subsubsection{Minimização do custo total (MCT)}

Outra maneira de abordar o problema é considerar a escolha de um tamanho amostral mínimo como um problema de decisão (Islam \& Pettit, 2014; Lindley, 1997; Parmigiani \& Inoue, 2009). Aqui, consideramos o custo de coleta de observações, que não levamos em conta (explicitamente) na abordagem anterior. Para tal propósito, é necessário especificar uma função perda $L\left(\lambda, d_{n}\right)$ baseada em uma amostra $X_{1}, \ldots, X_{n}$ e uma decisão $d_{n}$, quando o valor do parâmetro é $\lambda$.

Comumente, no problema de determinação do tamanho amostral a função perda tem a forma 
(vide e.g. Parmigiani \& Inoue, 2009)

$$
L\left(\lambda, d_{n}, n\right)=L\left(\lambda, d_{n}\right)+C(n),
$$

em que $C(n)=c n$ com $c$ representando o custo para obtenção de uma alíquota. Note que outras expressões para $C(n)$ podem ser consideradas, inclusive uma função que dependa também das observações desde que seja uma função crescente em $n$.

No caso de inferência intervalar, uma decisão corresponde à determinação de duas quantidades ( $a$ e $b, a \leq b)$ que formam uma região (ou intervalo) de credibilidade para o parâmetro de interesse (em nosso caso, a concentração média $\lambda$ ). Já no caso em que consideramos uma distribuição a priori não-paramétrica, uma decisão corresponde à determinação de uma função distribuição para o parâmetro de interesse.

Seja $F_{0}$ a distribuição a priori para o parâmetro desconhecido $\lambda$ e $d_{n}$ uma decisão, o risco de Bayes a priori é definido como (vide e.g. Parmigiani \& Inoue, 2009)

$$
r\left(F_{0}, d_{n}\right)=\int_{\Lambda} R\left(\lambda, d_{n}\right) F_{0}(d \lambda)
$$

em que $\Lambda$ é o espaço paramétrico e $R\left(\lambda, d_{n}\right)$ é a perda esperada amostral ou função de risco, definida como

$$
R\left(\lambda, d_{n}\right)=\int_{\mathcal{X}^{n}} L\left(\lambda, d_{n}\right) g\left(\boldsymbol{x}_{n} \mid \lambda\right) d \boldsymbol{x}_{n}
$$

O risco de Bayes a priori $r\left(F_{0}, d_{n}\right)$ pode ser visto como a média da perda esperada amostral (como uma função do parâmetro de interesse) ponderada pela distribuição a priori; isso é uma maneira de resumir a perda esperada amostral sobre os possíveis valores do parâmetro de interesse. A decisão $d_{n}^{*}$ que minimiza $r\left(F_{0}, d_{n}\right)$ entre todas possíveis decisões $d_{n}$ é chamada de decisão de Bayes. Mas note que

$$
r\left(F_{0}, d_{n}\right)=\int_{\Lambda}\left[\int_{\mathcal{X}^{n}} L\left(\lambda, d_{n}\right) g\left(\boldsymbol{x}_{n} \mid \lambda\right) d \boldsymbol{x}_{n}\right] F_{0}(d \lambda)=\int_{\mathcal{X}^{n}}\left[\int_{\Lambda} L\left(\lambda, d_{n}\right) F\left(d \lambda \mid \boldsymbol{x}_{n}\right)\right] g\left(\boldsymbol{x}_{n}\right) d \boldsymbol{x}_{n},
$$

supondo que a ordem de integração pode ser invertida. Desta forma, obter a decisão $d_{n}^{*}$ que minimiza $r\left(F_{0}, d_{n}\right)$ é equivalente a obter a decisão que minimiza o valor esperado a posteriori da função perda, ou $\mathbb{E}\left[L\left(\lambda, d_{n}\right) \mid \boldsymbol{x}_{\boldsymbol{n}}\right]$. Nesse contexto, o tamanho amostral desejado é aquele que minimiza o custo total

$$
C T(n)=r\left(F_{0}, d_{n}^{*}\right)+C(n) .
$$


Muitas vezes não é possível calcular $r\left(F_{0}, d_{n}^{*}\right)$ analiticamente. Então, podemos usar simulações de Monte Carlo para abordar o problema. Para um dado $n, r\left(F_{0}, d_{n}^{*}\right)$ é basicamente estimado da seguinte forma baseando-se na última expressão de (3.4): simule $\boldsymbol{x}_{n}$ e obtenha $d_{n}^{*}$, em seguida calcule $\mathbb{E}\left[L\left(\lambda, d_{n}^{*}\right) \mid \boldsymbol{x}_{\boldsymbol{n}}\right]$; repita o procedimento anterior (e.g., 1000 vezes), de forma a obter 1000 valores para o valor esperado a posteriori da perda. A média desses 1000 valores será a estimativa do risco de Bayes; posteriormente some $C(n)$ a essa estimativa. O processo anterior é repetido para outros valores de $n$ em um dado conjunto de maneira que obteremos pares de valores $(n, C T(n))$, como em problemas de regressão linear. O tamanho amostral $n$ desejado é obtido minimizando as estimativas de $C T(n)$ em relação a $n$ analiticamente. Neste caso, o conjunto de valores no qual $n$ irá variar é arbitrário. Por exemplo, podemos considerar $n=1,2, \ldots, n_{\max }$, em que $n_{\max }$ é um valor inteiro arbitrário (e.g., $\left.n_{\max }=100\right)$, ou $n=1,5,10, \ldots, 100$. Optamos pela última alternativa e adicionalmente calculamos mais de uma estimativa para cada valor $n$ de forma a gerar variabilidade nas estimativas de $C T(n)$.

Uma vez que métodos de simulação estão sendo usados, as estimativas de $C T(n)$ podem mostrar uma variação em torno do verdadeiro valor. Podemos reduzir essa variação da seguinte maneira: (1) tomando um número de réplicas de Monte Carlo tão grande quanto possível e, (2) além disso, ajustando uma curva (e.g., pelo método dos mínimos quadrados) às estimativas de $C T(n)$ como função de $n$. Müller \& Parmigiani (1995) propõem ajustar a seguinte curva às estimativas de $C T(n)$,

$$
C T(n)=\frac{E}{(1+H n)^{G}}+c n,
$$

em que $E, H$, e $G$ são parâmetros a serem estimados. A estimação desses parâmetros necessita métodos numéricos para os quais, às vezes, não há convergência dependendo dos valores iniciais. Como (1) os parâmetros $H$ e $G$ desempenham papéis similares (ou até o mesmo) e essencialmente representam uma espécie de taxa de decrescimento do risco de Bayes a priori, (2) levando em conta que a função $(1+n)^{G}$ oferece mais alternativas para uma taxa de decrescimento do que $1+H n$, e (3) dependendo do valor de $G$, um termo linear (como $1+H n$ ) pode ser incluído por meio da expansão dessa função em série de Taylor, propomos usar a seguinte função alternativa para o ajuste:

$$
C T(n)=\frac{E}{(1+n)^{G}}+c n .
$$


Essa função pode ser linearizada como

$$
\log [C T(n)-c n]=\log E-G \log (1+n)
$$

em que $-\log (1+n)$ pode ser interpretado como variável explicativa e $\log [C T(n)-c n]$, como variável dependente. Supondo que um erro aleatório seja adicionado, as estimativas de $E$ e $G$ podem ser calculadas por meio do método dos mínimos quadrados. Então, o tamanho amostral mínimo desejado é o inteiro mais próximo de

$$
\left(\frac{\widehat{E} \widehat{G}}{c}\right)^{1 /(\widehat{G}+1)}-1
$$

em que $\widehat{E}$ e $\widehat{G}$ são as estimativas de mínimos quadrados de $E$ e $G$, respectivamente.

Neste ponto precisamos definir as funções perda que serão utilizadas. Consideramos as duas seguintes funções perda para o problema no caso dos modelos (2.1)-(2.2) e (2.3)-(2.4).

\section{Função perda 1}

Com a finalidade de determinar o tamanho amostral mínimo para obter uma região (ou intervalo) de credibilidade para a concentração média de organismos na água de lastro, primeiramente consideramos a seguinte função perda

$$
L\left(\lambda, d_{n}\right)=\rho \tau+(a-\lambda)^{+}+(\lambda-b)^{+},
$$

em que $0<\rho<1$ é um peso, $\tau=(b-a) / 2$ é a semiamplitude do intervalo, a função $x^{+}$é igual a $x$ se $x>0$ e igual a 0 , em caso contrário e a decisão $d_{n}=d_{n}(a, b)$ corresponde à determinação dos limitantes de um intervalo de credibilidade. Quanto menor $\tau$, menor será o intervalo. Os termos $(a-\lambda)^{+} \mathrm{e}(\lambda-b)^{+}$são incluídos para penalizar os intervalos que não contêm o parâmetro de interesse $(\lambda)$. Esses termos são iguais 0 se $\lambda \in[a, b]$ e aumentam à medida que $\lambda$ se distancia dos respectivos intervalos. Note que a função perda (3.7) é a soma ponderada de dois termos, $\tau$ e $(a-\lambda)^{+}+(\lambda-b)^{+}$, em que os pesos são $\rho$ e 1, respectivamente. Nesse contexto, Rice et al. (2008) argumentam que o segundo termo da função perda deve receber maior peso, i.e., $\rho<1$. A correspondente decisão de Bayes corresponde ao intervalo formado pelos quantis $\rho / 2$ e $1-\rho / 2$ da distribuição a posteriori de $\lambda$. Mais detalhes e algoritmos para implementar o cálculo do tamanho amostral encontram-se em A.3.1, A.4.3 e B.3.3 nos Apêndices A e B, respectivamente. 


\section{Função perda 2}

Outra função perda que pode ser utilizada neste contexto é

$$
L\left(\lambda, d_{n}\right)=\gamma \tau+(\lambda-m)^{2} / \tau
$$

em que $\gamma>0$ é uma constante fixada e $m=(a+b) / 2$ é o centro do intervalo de credibilidade. Nessa função perda, o primeiro termo envolve a semiamplitude do intervalo e o segundo, o quadrado da distância entre o parâmetro de interesse e o centro do intervalo, que dividimos pela semiamplitude para manter a mesma unidade de medida do primeiro termo e assim torná-los comparáveis. Os pesos atribuídos a cada termo são $\gamma$ e 1 , respectivamente. Se $\gamma<1$, atribuímos maior peso ao segundo termo; se $\gamma>1$, a situação é invertida e se $\gamma=1$, os dois termos têm o mesmo peso. Nesse caso, a decisão de Bayes corresponde ao intervalo $[a, b]=\left[m-\mathrm{SD}_{\gamma}, m+\mathrm{SD}_{\gamma}\right]$, em que $\left(m, \mathrm{SD}_{\gamma}\right)=\left(\mathbb{E}\left[\lambda \mid \boldsymbol{x}_{n}\right], \gamma^{-1 / 2} \sqrt{\operatorname{Var}\left[\lambda \mid \boldsymbol{x}_{n}\right]}\right)$, vide e.g. Schervish (1995), Rice et al. (2008) e Parmigiani \& Inoue (2009). Mais detalhes e algoritmos para implementar o cálculo do tamanho amostral encontram-se em A.3.2, A.4.3 e B.3.3 nos Apêndices A e B, respectivamente.

\section{Função perda 3}

As funções perda 1 e 2 se adéquam bem aos modelos (2.1)-(2.2) e (2.3)-(2.4). Entretanto, quando consideramos o modelo (2.5)-(2.7) temos que levar em consideração que o espaço paramétrico é um conjunto de funções distribuição. Uma função perda utilizável neste caso é

$$
L\left(F, D_{n}\right)=\int_{-\infty}^{\infty}\left[F\left(A_{t}\right)-D_{n}\left(A_{t}\right)\right]^{2} W(d t)
$$

em que $A_{t}=(-\infty, t], W(d t)$ é uma função de ponderação tal que $\int_{-\infty}^{\infty} W(d t)=1$ e $D_{n}$ é uma função distribuição (Antoniak, 1974). Neste caso, a decisão será uma função distribuição que representamos por $D_{n}$ (por isso a representação em letra maiúscula ao invés de minúscula como nas funções perda anteriores).

O risco de Bayes para o problema "sem amostra" é calculado tomando-se o valor esperado em relação ao processo Dirichlet fixado como distribuição a priori, i.e.,

$$
\mathbb{E}\left[L\left(F, D_{0}\right)\right]=\int_{-\infty}^{\infty} \mathbb{E}\left[\left(F\left(A_{t}\right)-D_{0}\left(A_{t}\right)\right)^{2}\right] W(d t)
$$

Logo, a decisão de Bayes neste caso será $D_{0}^{*}\left(A_{t}\right)=\mathbb{E}\left[F\left(A_{t}\right)\right]=F_{0}\left(A_{t}\right)$. Dadas observações resultantes 
de uma mistura por processo Dirichlet, então a decisão de Bayes para o problema com uma amostra de tamanho $n$ é

$$
D_{n}^{*}\left(A_{t}\right)=\mathbb{E}\left[F\left(A_{t} \mid \boldsymbol{x}_{n}\right)\right],
$$

pois $F\left(A_{t} \mid \boldsymbol{\lambda}_{n}\right)$ é um processo Dirichlet e $F\left(A_{t} \mid \boldsymbol{x}_{n}\right)$ é uma mistura de processos Dirichlet (Antoniak, 1974, pp. 1159 e 1160). Mais detalhes e algoritmos para implementar o cálculo do tamanho amostral encontram-se em C.2.1 e C.3.7 no Apêndice C. 


\section{Capítulo 4}

\section{Resultados e discussão}

Apresentamos metodologias que permitem obter o tamanho amostral mínimo necessário para estimar a concentração média de organismos no tanque de lastro sob alguns modelos, possibilitando assim verificar se um navio cumpre a norma D-2 ou não. Optamos por inferência intervalar seguindo a sugestão de Costa et al. (2016) para os modelos (2.1)-(2.2) e (2.3)-(2.4). Neste contexto, declaramos o não cumprimento da norma se $a>10$, seu cumprimento, se $b<10$, em que $a$ e $b$ são os limitantes inferior e superior, respectivamente, do intervalo de credibilidade obtido. Se $a<10<b$ precisaremos de mais informações para tomar uma decisão. No caso do modelo (2.5)-(2.7), a inferência será baseada em uma região do espaço paramétrico (que pode ser a união de dois ou mais intervalos); declararemos o não cumprimento da norma se os limitantes inferiores de todos os intervalos que compõem a região forem maiores do que 10; a norma estará cumprida se os limitantes superiores de todos os intervalos que compõem a região forem menores do que 10. Se nem todos os limitantes inferiores (superiores) forem maiores do que (menores do que) 10 precisaremos de mais informação para tomar uma decisão.

\subsection{Abordagem baseada nos critérios CCobM e CCompM}

Nas Tabelas 4.1 e 4.2 encontram-se os tamanhos amostrais mínimos calculados sob o critério CCobM (3.1) para os modelos (2.1)-(2.2) e (2.3)-(2.4). Note que no caso do modelo (2.3)-(2.4) consideramos $\lambda_{0}=10(w / \phi)$ de modo que o valor esperado a priori fosse igual a 10 para permitir a comparação com o modelo (2.1)-(2.2) com $\lambda_{0}=10$.

Considerando a Tabela 4.1, o tamanho amostral não diminui muito mesmo quando $\theta_{0}$ aumenta, i.e., quando a variância a priori diminui. Isso pode ser justificado pela suposição de homogeneidade da concentração inerente ao modelo adotado. A menos que o conhecimento a priori seja bastante 
preciso, o tamanho amostral mínimo necessário para satisfazer o CCobM não sofrerá muita alteração. Essa característica também é visível quando calculamos o tamanho amostral sob o mesmo modelo com o critério CCompM (vide Tabela 4.4).

Por outro lado, sob o modelo (2.3)-(2.4) usando os critérios CCobM e CCompM com um valor de $\phi$ fixado, a precisão do conhecimento a priori, neste caso controlada por $\theta_{0}$, influencia diretamente o tamanho amostral mínimo. O mesmo acontece quando consideramos $\theta_{0}$ fixo e $\phi$ é incrementado, pois $\phi$ desempenha o papel de parâmetro de escala na distribuição a priori (vide Tabelas 4.2 e 4.6). Devemos destacar que $\phi$ desempenha dois papéis no modelo (2.3)-(2.4). Em (2.3), desempenha o papel de parâmetro de dispersão (ou aglomeração dos organismos no tanque). Quanto maior $\phi$, mais homogênea é a concentração dos organismos no tanque. Na distribuição a priori $(2.4), \phi$ desempenha um papel de parâmetro de escala. Quanto maior $\phi$ (considerando os outros parâmetros fixos), menos preciso é o conhecimento a priori sobre a concentração média (vide Figura 2.2). Isso não quer dizer que se $\phi$ (conhecido previamente) for grande só poderemos usar distribuições a priori com grande variabilidade, pois podemos especificar os parâmetros $\lambda_{0}$ e $\theta_{0}$ para ajustar a precisão do conhecimento a priori mesmo com valores grandes de $\phi$ (vide Figura 2.3).

Para o critério CCompM apresentamos um resultado (Teorema 3.1) que permite o cálculo do tamanho amostral mínimo sob o modelo (2.1)-(2.2). O maior atrativo desse resultado é que não necessitamos recorrer a simulações de Monte Carlo para estimar as quantidades necessárias no processo de obtenção do tamanho amostral. Os tamanhos amostrais dispostos na Tabela 4.5 (em que o Teorema 3.1 foi utilizado) são similares àqueles apresentados na Tabela 4.4; no entanto, aqueles calculados por meio do Teorema 3.1 são sempre menores do que ou iguais àqueles apresentados na Tabela 4.4.

Tabela 4.1: Número mínimo de alíquotas (n) com informação a priori $\lambda_{0}=10$ sob o modelo (2.1)-(2.2) com o critério CCobM (3.1) e $\rho=0,05$.

\begin{tabular}{ccrrrrr}
\hline Volume da & Tamanho do & \multicolumn{5}{c}{ Parâmetro de forma $\left(\theta_{0}\right)$} \\
\cline { 2 - 7 } alíquota $(w)$ & intervalo $(\ell)$ & 1,0 & 2,5 & 5,0 & 7,5 & 10,0 \\
\hline \multirow{2}{*}{0,5} & 2 & 77 & 77 & 76 & 76 & 75 \\
& 4 & 20 & 19 & 19 & 18 & 18 \\
\cline { 2 - 7 } 1,0 & 2 & 39 & 39 & 38 & 38 & 38 \\
& 4 & 10 & 10 & 10 & 9 & 9 \\
\hline
\end{tabular}

Segundo Joseph \& Bélisle (1997), tamanhos amostrais calculados sob o critério CCompM tendem a ser menores que aqueles calculados sob o critério CCobM quando $1-\rho$ está próximo de 1 e $\ell$ $\left(\ell_{\max }\right)$ não está próximo de 0 . Esses autores explicam que isso acontece porque as probabilidades de cobertura bayesiana são limitadas superiormente por 1 e a manutenção desejada para a cobertura 
Tabela 4.2: Número mínimo de alíquotas ( $n$ ) com informação a priori $\lambda_{0}=10(w / \phi)$ sob o modelo (2.3)-(2.4) com o critério CCobM (3.1) e $\rho=0,05$.

\begin{tabular}{|c|c|c|c|c|c|c|}
\hline \multirow{2}{*}{$\begin{array}{l}\text { Volume da } \\
\text { alíquota }(w)\end{array}$} & \multirow{2}{*}{$\begin{array}{l}\text { Tamanho do } \\
\text { intervalo }(\ell)\end{array}$} & \multirow{2}{*}{$\phi$} & \multicolumn{4}{|c|}{ Parâmetro de forma $\left(\theta_{0}\right)$} \\
\hline & & & 11 & 25 & 50 & 75 \\
\hline \multirow{10}{*}{0,5} & \multirow{5}{*}{2} & 1,0 & 462 & 457 & 453 & 444 \\
\hline & & 2,5 & 229 & 226 & 222 & 216 \\
\hline & & 5,0 & 152 & 149 & 144 & 140 \\
\hline & & 7,5 & 127 & 124 & 118 & 114 \\
\hline & & 10,0 & 113 & 111 & 106 & 101 \\
\hline & \multirow{5}{*}{4} & 1,0 & 115 & 112 & 106 & 101 \\
\hline & & 2,5 & 57 & 53 & 49 & 43 \\
\hline & & 5,0 & 37 & 34 & 29 & 24 \\
\hline & & 7,5 & 30 & 28 & 22 & 17 \\
\hline & & 10,0 & 27 & 24 & 19 & 14 \\
\hline \multirow{10}{*}{1,0} & \multirow{5}{*}{2} & 1,0 & 426 & 422 & 417 & 414 \\
\hline & & 2,5 & 194 & 191 & 188 & 185 \\
\hline & & 5,0 & 115 & 113 & 111 & 108 \\
\hline & & 7,5 & 90 & 88 & 85 & 82 \\
\hline & & 10,0 & 77 & 75 & 72 & 70 \\
\hline & \multirow{5}{*}{4} & 1,0 & 110 & 107 & 102 & $\overline{99}$ \\
\hline & & 2,5 & 49 & 46 & 44 & 41 \\
\hline & & 5,0 & 29 & 27 & 24 & 22 \\
\hline & & 7,5 & 22 & 20 & 18 & 15 \\
\hline & & 10,0 & 19 & 17 & 15 & 12 \\
\hline
\end{tabular}

média se torna cada vez mais custosa à medida que $1-\rho$ cresce. Similarmente, dado que $\ell\left(\ell_{\max }\right)$ é limitado inferiormente por 0, a manutenção de um tamanho médio desejado se torna cada vez mais custosa à medida que $\ell\left(\ell_{\max }\right)$ se aproxima de 0 . Como evidências para suas afirmações, Joseph \& Bélisle (1997) calculam tamanhos amostrais sob ambos os critérios em seis cenários para o problema de estimação da média do modelo normal com variância desconhecida. São considerados os valores 0,2 e 0,5 para $\ell\left(\ell_{\max }\right)$, e 0,01, 0,05, 0,20 e 0,50 para $\rho$. Em quatro dos seis cenários, as afirmações dos autores são corroboradas; nos dois restantes, os tamanhos amostrais obtidos sob ambos os critérios são similares.

Joseph et al. (1995) consideram vários cenários para o problema de estimação do parâmetro de um modelo binomial com mais valores fixados para $\ell\left(\ell_{\max }\right)$ e $\rho$, e chegam às mesmas evidências que Joseph \& Bélisle (1997). Em alguns cenários, os respectivos tamanhos amostrais calculados usando os critérios CCobM e CCompM são bem diferentes, enquanto em outros cenários os respectivos tamanhos amostrais destes critérios são similares. Vale ressaltar que Joseph \& Bélisle (1997) consideram dois valores para os hiperparâmetros no cálculo do tamanho amostral, enquanto Joseph et al. (1995) consideram uma distribuição a priori uniforme entre outros valores para os hiperparâmetros. Note também que no caso de Joseph et al. (1995), $\ell$ ( $\left.\ell_{\max }\right)$ é limitado superiormente 
Tabela 4.3: Número mínimo de alíquotas (n) usando o critério CCobM sob o modelo (2.5)-(2.7) com $F_{0}=G\left(\theta_{0}, \theta_{0} / \lambda_{0}\right), \lambda_{0}=10$ e $\rho=0,05$.

\begin{tabular}{|c|c|c|c|c|c|c|c|}
\hline \multirow{2}{*}{$\begin{array}{l}\text { Volume da } \\
\text { alíquota }(w)\end{array}$} & \multirow{2}{*}{$\begin{array}{l}\text { Tamanho da } \\
\text { região }(\ell)\end{array}$} & \multirow{2}{*}{$\alpha^{*}$} & \multicolumn{5}{|c|}{ Parâmetro de forma $\left(\theta_{0}\right)$} \\
\hline & & & 1,0 & 2,5 & 5,0 & 7,5 & 10,0 \\
\hline \multirow{10}{*}{0,5} & \multirow{5}{*}{2} & 0,5 & 8 & 8 & 8 & 8 & 7 \\
\hline & & 1,5 & 26 & 26 & 24 & 23 & 23 \\
\hline & & 2,5 & 54 & 52 & 48 & 45 & 44 \\
\hline & & 5,0 & 176 & 168 & 156 & 149 & 143 \\
\hline & & 10,0 & 644 & 628 & 588 & 554 & 529 \\
\hline & \multirow{5}{*}{4} & 0,5 & 7 & 7 & 6 & 6 & 5 \\
\hline & & 1,5 & 20 & 21 & 19 & 17 & 15 \\
\hline & & 2,5 & 36 & 36 & 32 & 29 & 26 \\
\hline & & 5,0 & 88 & 83 & 73 & 65 & 60 \\
\hline & & 10,0 & 280 & 269 & 236 & 213 & 190 \\
\hline \multirow{10}{*}{1,0} & \multirow{5}{*}{2} & 0,5 & 8 & 8 & 8 & 8 & 7 \\
\hline & & 1,5 & 28 & 26 & 25 & 24 & 23 \\
\hline & & 2,5 & 58 & 54 & 49 & 47 & 44 \\
\hline & & 5,0 & 183 & 174 & 162 & 151 & 143 \\
\hline & & 10,0 & 654 & 636 & 594 & 563 & 533 \\
\hline & \multirow{5}{*}{4} & 0,5 & 7 & 7 & 6 & 5 & 5 \\
\hline & & 1,5 & 20 & 21 & 19 & 17 & 15 \\
\hline & & 2,5 & 36 & 36 & 32 & 29 & 27 \\
\hline & & 5,0 & 90 & 85 & 74 & 66 & 60 \\
\hline & & 10,0 & 289 & 274 & 238 & 214 & 193 \\
\hline
\end{tabular}

Tabela 4.4: Número mínimo de alíquotas (n) com informação a priori $\lambda_{0}=10$ sob o modelo (2.1)-(2.2) com o critério CCompM (3.3) e $\rho=0,05$.

\begin{tabular}{ccrrrrr}
\hline Volume da & Tamanho máximo & \multicolumn{5}{c}{ Parâmetro de forma $\left(\theta_{0}\right)$} \\
\cline { 3 - 7 } alíquota $(w)$ & do intervalo $\left(\ell_{\max }\right)$ & 1,0 & 2,5 & 5,0 & 7,5 & 10,0 \\
\hline \multirow{2}{*}{0,5} & 2 & 77 & 77 & 76 & 76 & 75 \\
& 4 & 19 & 19 & 19 & 18 & 17 \\
\cline { 2 - 7 } 1,0 & 2 & 38 & 38 & 38 & 38 & 38 \\
& 4 & 10 & 10 & 9 & 9 & 9 \\
\hline
\end{tabular}

e inferiormente como $\rho$, pois o parâmetro de interesse é uma probabilidade.

Rahme et al. (2000) comentam que os critérios CCobM e CCompM frequentemente fornecem tamanhos amostrais similares, mas não apresentam evidências sobre isso. Em nosso caso, os tamanhos amostrais calculados usando o CCobM ou o CCompM sob o mesmo modelo são similares. De qualquer forma, um estudo que considere uma ampla variedade de modelos e de valores para os hiperparâmetros além de $\rho$ e $\ell\left(\ell_{\max }\right)$ seria necessário para chegarmos mais próximos de um consenso. Esse estudo ou até mesmo uma demonstração analítica são tópicos para futuras pesquisas.

Os tamanhos amostrais calculados sob ambos os critérios mostram evidências de que os cálculos obtidos com o CCobM são computacionalmente mais rápidos do que aqueles obtidos com o CCompM. 
Tabela 4.5: Número mínimo de alíquotas ( $n$ ) com informação a priori $\lambda_{0}=10$ usando o Teorema 3.1 sob o modelo (2.1)-(2.2) e $\rho=0,05$.

\begin{tabular}{ccrrrrr}
\hline \multirow{2}{*}{$\begin{array}{c}\text { Volume da } \\
\text { alíquota }(w)\end{array}$} & Tamanho máximo & \multicolumn{5}{c}{ Parâmetro de forma $\left(\theta_{0}\right)$} \\
\cline { 3 - 7 } & do intervalo $\left(\ell_{\max }\right)$ & 1,0 & 2,5 & 5,0 & 7,5 & 10,0 \\
\hline \multirow{2}{*}{0,5} & 2 & 61 & 70 & 73 & 73 & 73 \\
& 4 & 15 & 17 & 18 & 18 & 17 \\
\cline { 2 - 7 } 1,0 & 2 & 31 & 35 & 37 & 37 & 37 \\
& 4 & 8 & 9 & 9 & 9 & 9 \\
\hline
\end{tabular}

Tabela 4.6: Número mínimo de alíquotas ( $n$ ) com informação a priori $\lambda_{0}=10(w / \phi)$ sob o modelo (2.3)-(2.4) com o critério CCompM (3.3) e $\rho=0,05$.

\begin{tabular}{ccrrrrr}
\hline Volume da & Tamanho máximo & & \multicolumn{5}{c}{ Parâmetro de forma $\left(\theta_{0}\right)$} \\
\cline { 3 - 7 } alíquota $(w)$ & do intervalo $\left(\ell_{\max }\right)$ & $\phi$ & 11 & 25 & 50 & 75 \\
\hline \multirow{3}{*}{0,5} & & 1,0 & 456 & 456 & 452 & 445 \\
& & 2,5 & 228 & 226 & 221 & 216 \\
& & 5,0 & 151 & 150 & 144 & 138 \\
& & 7,5 & 126 & 123 & 118 & 113 \\
& & 10,0 & 114 & 110 & 106 & 101 \\
\cline { 3 - 7 } & & 1,0 & 113 & 109 & 105 & 99 \\
& & 2,5 & 55 & 52 & 48 & 43 \\
& & 5,0 & 37 & 34 & 29 & 23 \\
& & 7,5 & 30 & 27 & 22 & 17 \\
& & 10,0 & 27 & 24 & 19 & 14 \\
\hline \multirow{3}{*}{2} & 1,0 & 414 & 418 & 416 & 410 \\
& & 2,5 & 191 & 190 & 186 & 185 \\
& & 5,0 & 115 & 113 & 110 & 108 \\
& & 7,5 & 89 & 88 & 85 & 82 \\
& & 10,0 & 76 & 75 & 72 & 70 \\
\hline \multirow{3}{*}{2} & 1,0 & 103 & 104 & 100 & 97 \\
& & 2,5 & 47 & 46 & 43 & 41 \\
& & 5,0 & 28 & 26 & 24 & 22 \\
& & 7,5 & 21 & 20 & 18 & 15 \\
& & 10,0 & 19 & 17 & 15 & 12 \\
\hline
\end{tabular}

Uma possível explicação pode ser o fato de que no CCobM, o tamanho amostral é incrementado até atingir-se uma probabilidade mínima fixada $(1-\rho)$, enquanto no CCompM, o tamanho amostral é incrementado até atingir-se um valor positivo máximo fixado $\left(\ell_{\max }\right)$. Uma busca no intervalo $(0,1)$ pode ser mais rápida do que uma busca em $(0, \infty)$. Dessa forma, o critério CCobM parece ser mais razoável sob o ponto de vista computacional. Como sugerido por M'Lan et al. (2006) e M'Lan et al. (2008), uma maneira possível de reduzir o custo computacional sob o CCompM é considerar a abordagem de ajuste de um modelo de regressão às estimativas geradas pelas simulações de Monte Carlo (semelhante àquela utilizada nesta tese na abordagem baseada na Teoria da Decisão). De acordo com (A.4), temos $p(n)=\mathbb{E}\left[\ell^{\prime}\left(\boldsymbol{X}_{n}, n\right)\right]=O\left(n^{-1 / 2}\right)$ sob o modelo (2.1)-(2.2). Assim, podemos 
Tabela 4.7: Número mínimo de alíquotas (n) usando o critério CCompM sob o modelo (2.5)-(2.7) com $F_{0}=G\left(\theta_{0}, \theta_{0} / \lambda_{0}\right), \lambda_{0}=10$ e $\rho=0,05$.

\begin{tabular}{|c|c|c|c|c|c|c|c|}
\hline \multirow{2}{*}{$\begin{array}{l}\text { Volume da } \\
\text { alíquota }(w)\end{array}$} & \multirow{2}{*}{$\begin{array}{l}\text { Tamanho máximo } \\
\text { da região }\left(\ell_{\max }\right)\end{array}$} & \multirow{2}{*}{$\alpha^{*}$} & \multicolumn{5}{|c|}{ Parâmetro de forma $\left(\theta_{0}\right)$} \\
\hline & & & 1,0 & 2,5 & 5,0 & 7,5 & 10,0 \\
\hline \multirow{10}{*}{0,5} & \multirow{5}{*}{2} & 0,5 & 8 & 8 & 8 & 8 & 8 \\
\hline & & 1,5 & 26 & 25 & 24 & 24 & 23 \\
\hline & & 2,5 & 54 & 53 & 49 & 46 & 44 \\
\hline & & 5,0 & 175 & 168 & 158 & 150 & 142 \\
\hline & & 10,0 & 643 & 628 & 583 & 553 & 528 \\
\hline & \multirow{5}{*}{4} & 0,5 & 7 & 7 & 6 & 6 & 5 \\
\hline & & 1,5 & 20 & 21 & 19 & 17 & 15 \\
\hline & & 2,5 & 36 & 36 & 32 & 29 & 26 \\
\hline & & 5,0 & 86 & 83 & 73 & 65 & 59 \\
\hline & & 10,0 & 283 & 268 & 237 & 210 & 191 \\
\hline \multirow{10}{*}{1,0} & \multirow{5}{*}{2} & 0,5 & 8 & 8 & 8 & 8 & 8 \\
\hline & & 1,5 & 26 & 26 & 25 & 24 & 23 \\
\hline & & 2,5 & 59 & 54 & 50 & 46 & 44 \\
\hline & & 5,0 & 182 & 173 & 162 & 153 & 143 \\
\hline & & 10,0 & 660 & 638 & 595 & 560 & 534 \\
\hline & \multirow{5}{*}{4} & 0,5 & 6 & 7 & 6 & 6 & 5 \\
\hline & & 1,5 & 20 & 21 & 19 & 17 & 15 \\
\hline & & 2,5 & 36 & 36 & 32 & 29 & 27 \\
\hline & & 5,0 & 91 & 86 & 73 & 66 & 60 \\
\hline & & 10,0 & 286 & 275 & 240 & 214 & 192 \\
\hline
\end{tabular}

ajustar o seguinte modelo de regressão

$$
[\widehat{p(n)}]^{-2}=E+G n+\text { erro, }
$$

em que $\widehat{p(n)}$ é uma estimativa de $p(n)$ obtida por simulações de Monte Carlo, $E$ e $G$ são parâmetros a serem estimados. Obtemos o tamanho amostral mínimo desejado resolvendo a equação $\widehat{E}+\widehat{G} n=$ $1 / \ell_{\max }^{2}$ em n, i.e.,

$$
n=\frac{1}{\widehat{G}}\left[\frac{1}{\ell_{\max }^{2}}-\widehat{E}\right]
$$

em que $\widehat{E}$ e $\widehat{G}$ são estimativas de $E$ e $G$ obtidas no ajuste do modelo de regressão. Para outros modelos, necessitaríamos um resultado similar a (A.4). A obtenção de tal resultado para outros modelos, bem como a análise dessa metodologia, é um tópico interessante para futuras pesquisas.

Os tamanhos amostrais obtidos sob a abordagem bayesiana com $\ell$ ou $\ell_{\max }$ (Tabelas $4.2 \mathrm{e}$ 4.6) são em geral menores do que aqueles calculados sob um enfoque frequentista com $\epsilon_{a}$ (erro absoluto máximo de estimação) igual a 1 ou 2 apresentados nas Tabelas 2 e 3 de Costa et al. (2016). Nesta última abordagem é possível utilizar alguma informação a priori com a atribuição de limites inferior e superior para o parâmetro de interesse, diferente da abordagem bayesiana que atribui 
uma distribuição de probabilidade.

Os tamanhos amostrais dispostos nas Tabelas 4.3 e 4.7 são similares quando comparados os respectivos cenários, como ocorreu com os modelos anteriores. Quando o parâmetro de forma $\theta_{0}$ aumenta, o tamanho amostral diminui consideravelmente para valores altos de $\alpha^{*}$. Note que para $\alpha^{*}$ pequeno, o correspondente tamanho amostral também é pequeno e à medida que $\alpha^{*}$ aumenta, o tamanho amostral também aumenta; o que está de acordo com um resultado provado por Sethuraman \& Tiwari (1981), que basicamente mostra que quanto menor o valor de $\alpha^{*}$, mais informação sobre a função distribuição que segue um processo Dirichlet e sua respectiva variável teremos (vide Apêndice C para mais detalhes).

\subsection{Abordagem baseada na Teoria da Decisão}

Os tamanhos amostrais calculados sob a função perda 1 são bem menores do que aqueles calculados sob a função perda 2 (vide Tabelas 4.8, 4.9, 4.10 e 4.11). Uma possível explicação está no fato de que quando consideramos $\rho=0,05$ na função perda 1 , estamos atribuindo menor peso ao tamanho do intervalo de credibilidade e peso maior (igual a 1) ao fato de o parâmetro $\lambda$ pertencer ao intervalo de credibilidade. Consequentemente, a amplitude do intervalo de credibilidade poderá ser maior, resultando em tamanhos amostrais menores. Por outro lado, quando consideramos a função perda 2 com $\gamma=1$, estamos atribuindo o mesmo peso tanto para a amplitude do intervalo de credibilidade quanto para a distância entre o parâmetro $\lambda$ e o centro do intervalo; mesmo no caso em que $\gamma=1 / 4$, quando maior peso é atribuído ao segundo termo, esta função perda parece fornecer intervalos de credibilidade mais conservadores. Consequentemente, tamanhos amostrais maiores serão necessários.

Os resultados dispostos na Tabela 4.9, obtidos sob o modelo (2.3)-(2.4) e função perda 1 , mostram que o tamanho amostral aumenta com o parâmetro $\phi$. Uma possível explicação pode ser o fato de $\phi$ ser um parâmetro de escala na distribuição a priori; quanto maior $\phi$, maior a incerteza ou variabilidade a priori a respeito de $\lambda$. Adicionalmente, o fato de essa função perda fornecer os quantis da distribuição a posteriori como componentes para inferência intervalar pode ser outra explicação. Se consideramos a função perda 2, isso não acontece, talvez pelo conservadorismo do intervalo de credibilidade associado (média a posteriori $\mp$ desvio padrão a posteriori), vide Tabela 4.11 .

A abordagem com o critério CCompM não inclui explicitamente uma função custo como na abordagem baseada na Teoria da Decisão. Entretanto, podemos argumentar que existe uma 
relação implícita entre o CCompM e o custo da amostragem. Uma diminuição no valor de $\ell_{\max }$ implicará mais observações necessárias e, consequentemente, um aumento no custo. Se houver um aumento no valor de $\ell_{\max }$, teremos uma diminuição no custo (Lindley, 1997). Lindley (1997) faz um paralelo interessante entre o CCompM e a abordagem baseada na Teoria da Decisão com uma determinada função perda. Seguindo a ideia de Lindley e usando a função perda 1, podemos argumentar da seguinte forma: considere que o parâmetro de interesse pertence ao intervalo $(a, b) \mathrm{e}$ que o tamanho esperado do intervalo pode ser representado por uma função de $n$. Então, escreva $\mathbb{E}\left[\ell^{\prime}\left(\boldsymbol{X}_{n}, n\right)\right]=p(n)$ e observe que na abordagem baseada na Teoria da Decisão devemos minimizar a expressão $\rho p(n) / 2+c n$. Derivando essa expressão em relação a $n$ e igualando a zero temos que

$$
p^{\prime}(n)=-\frac{2 c}{\rho},
$$

enquanto no critério CCompM igualamos o tamanho esperado do intervalo a $\ell$, i.e.,

$$
p(n)=\ell .
$$

Suponha que as duas abordagens concordem para algum $c$ e $\ell$, de maneira que (4.1) e (4.2) tenham o mesmo valor $n$ como solução. Derivando os dois membros da equação (4.2) em relação a $\ell$ temos que

$$
\frac{\partial n}{\partial \ell}=\frac{1}{p^{\prime}(n)},
$$

e da equação (4.1) obtemos

$$
\frac{\partial n}{\partial \ell}=\frac{-\rho}{2 c}
$$

Então, uma variação $\Delta$ em $\ell$ irá implicar uma variação de $-\Delta \rho / 2 c$ em $n$. Por exemplo, considere $\rho=0,05$ e $c=0,005$; então uma diminuição de 1 unidade em $\ell$ implica o aumento de cinco observações na amostra.

Na Tabela 4.12, comparando os valores para $w=0,5$ e $w=1$ notamos que são bastante similares, implicando que o tamanho amostral não é influenciado pelo volume da alíquota neste caso. Os tamanhos amostrais obtidos com essa função perda são consideravelmente menores do que aqueles obtidos sob o mesmo modelo na abordagem baseada nos critérios CCobM e CCompM. Uma explicação pode ser o fato de que nessa função perda não é imposto nenhum limitante (majorante ou minorante) para o tamanho da região HPD e para a sua probabilidade. Neste caso é minimizada a "proximidade" entre a função distribuição $D_{n}^{*}$ e a função distribuição do parâmetro de interesse, 
calculada por meio de uma função quadrática.

Tabela 4.8: Número mínimo de alíquotas (n) com informação a priori $\lambda_{0}=10$ sob o modelo (2.1)-(2.2) com a função perda 1 e $\rho=0,05$.

\begin{tabular}{ccrrrrr}
\hline Volume da & Custo de uma & \multicolumn{5}{c}{ Parâmetro de forma $\left(\theta_{0}\right)$} \\
\cline { 3 - 7 } alíquota $(w)$ & alíquota $(c)$ & 1,0 & 2,5 & 5,0 & 7,5 & 10,0 \\
\hline \multirow{2}{*}{0,5} & 0,005 & 14 & 14 & 13 & 13 & 12 \\
& 0,010 & 9 & 9 & 8 & 8 & 8 \\
\cline { 2 - 7 } 1,0 & 0,005 & 11 & 11 & 11 & 11 & 11 \\
& 0,010 & 7 & 7 & 7 & 7 & 6 \\
\hline
\end{tabular}

Tabela 4.9: Número mínimo de alíquotas $(n)$ com informação a priori $\lambda_{0}=10(w / \phi)$ sob o modelo (2.3)-(2.4) com a função perda 1 e $\rho=0,05$.

\begin{tabular}{|c|c|c|c|c|c|c|}
\hline \multirow{2}{*}{$\begin{array}{l}\text { Volume da } \\
\text { alíquota }(w)\end{array}$} & \multirow{2}{*}{$\begin{array}{l}\text { Custo de uma } \\
\text { alíquota }(c)\end{array}$} & \multirow{2}{*}{$\phi$} & \multicolumn{4}{|c|}{ Parâmetro de forma $\left(\theta_{0}\right)$} \\
\hline & & & 11 & 25 & 50 & 75 \\
\hline \multirow{10}{*}{0,5} & \multirow{5}{*}{0,005} & 1,0 & 9 & 7 & 5 & 4 \\
\hline & & 2,5 & 12 & 10 & 7 & 6 \\
\hline & & 5,0 & 16 & 14 & 11 & 9 \\
\hline & & 7,5 & 20 & 17 & 14 & 11 \\
\hline & & 10,0 & 24 & 20 & 16 & 14 \\
\hline & \multirow{5}{*}{0,010} & 1,0 & 5 & 4 & 3 & 2 \\
\hline & & 2,5 & 7 & 6 & 4 & 3 \\
\hline & & 5,0 & 10 & 8 & 6 & 5 \\
\hline & & 7,5 & 12 & 10 & 8 & 6 \\
\hline & & 10,0 & 15 & 12 & 10 & 8 \\
\hline \multirow{10}{*}{1,0} & \multirow{5}{*}{0,005} & 1,0 & 6 & 5 & 4 & 3 \\
\hline & & 2,5 & 8 & 7 & 5 & 5 \\
\hline & & 5,0 & 10 & 9 & 7 & 6 \\
\hline & & 7,5 & 12 & 11 & 9 & 8 \\
\hline & & 10,0 & 14 & 12 & 10 & 9 \\
\hline & \multirow{5}{*}{0,010} & 1,0 & 4 & 3 & 2 & 2 \\
\hline & & 2,5 & 5 & 4 & 3 & 3 \\
\hline & & 5,0 & 6 & 5 & 4 & 4 \\
\hline & & 7,5 & 7 & 6 & 5 & 5 \\
\hline & & 10.0 & 8 & 7 & 6 & 5 \\
\hline
\end{tabular}

\subsection{Representação da informação a priori}

Uma parte importante da abordagem bayesiana é a incorporação de conhecimento a priori traduzido na forma de uma distribuição de probabilidade. Dessa forma surge um problema que é a determinação de uma distribuição de probabilidade que reflita tal conhecimento, quando houver. Note que para os tamanhos amostrais apresentados nas tabelas deste capítulo foi considerado que a média da distribuição a priori é igual a 10, o que poderíamos dizer que seria a situação "limite" entre o cumprimento e o não cumprimento da norma D-2. Ao invés disso, poderíamos considerar 
Tabela 4.10: Número mínimo de alíquotas ( $n$ ) com informação a priori $\lambda_{0}=10$ sob o modelo (2.1)-(2.2) com a função perda 2.

\begin{tabular}{cccccccc}
\hline \multirow{2}{*}{$\begin{array}{c}\text { Volume da } \\
\text { alíquota }(w)\end{array}$} & $\begin{array}{c}\text { Custo de uma } \\
\text { alíquota }(c)\end{array}$ & $\gamma$ & \multicolumn{5}{c}{ Parâmetro de forma $\left(\theta_{0}\right)$} \\
\cline { 4 - 9 } 0,5 & \multirow{2}{*}{0,005} & 1 & 94 & 94 & 92 & 90 & 89 \\
& & $1 / 4$ & 60 & 59 & 58 & 56 & 55 \\
\cline { 2 - 8 } 0 & \multirow{2}{*}{0,010} & 1 & 60 & 59 & 58 & 56 & 55 \\
& & $1 / 4$ & 38 & 37 & 36 & 35 & 34 \\
\hline \multirow{3}{*}{1,0} & \multirow{2}{*}{0,005} & 1 & 75 & 75 & 75 & 74 & 73 \\
& \multirow{2}{*}{0,010} & $1 / 4$ & 48 & 48 & 47 & 46 & 46 \\
\cline { 3 - 8 } & & $1 / 4$ & 48 & 48 & 47 & 46 & 46 \\
& & & & 30 & 30 & 29 & 29 \\
\hline
\end{tabular}

um valor para a média a priori abaixo de 10, i.e., uma abordagem do "inocente até que se prove o contrário" (Miller et al., 2011). De qualquer forma, ainda restaria estabelecer (ou estimar) valores para as quantidades $\theta_{0}, \phi$ e $\alpha^{*}$.

Uma maneira de estabelecer um valor para $\alpha^{*}$ é considerar a situação "limite" entre o cumprimento e o não cumprimento da norma D-2 considerando que o valor esperado da probabilidade da concentração ser menor do que ou igual a 10 (cumprimento) seja igual a 1/2. Então, podemos escrever

$$
\mathbb{E}\left[F\left(A_{10}\right)\right]=F_{0}\left(A_{10}\right)=\frac{1}{2}
$$

em que $A_{10}=(-\infty, 10]$. Se fixarmos uma variabilidade $\eta \leq 1 / 4$ para essa probabilidade, temos que

$$
\operatorname{Var}\left[F\left(A_{10}\right)\right]=\frac{F_{0}\left(A_{10}\right)\left[1-F_{0}\left(A_{10}\right)\right]}{\alpha^{*}+1}=\frac{1}{4\left(\alpha^{*}+1\right)}=\eta
$$

de onde obtemos $\alpha^{*}=(4 \eta)^{-1}-1$.

Se considerarmos $\phi$ desconhecido, devemos então atribuir uma distribuição a priori para este parâmetro que provavelmente terá hiperparâmetros. Tais hiperparâmetros (assim como $\theta_{0}$ ) podem ser eliciados por meio dos momentos ou quantis da distribuição a priori. Neste ponto, o problema que surge é a origem do conhecimento a priori que será utilizado para estabelecer uma distribuição de probabilidade.

Segundo Aguirre-Macedo et al. (2008), é importante considerar a origem da água de lastro na hora de avaliar o possível impacto do seu deslastre. Ainda segundo esses autores, o risco de introdução de organismos é baixo se a água de lastro é de origem oceânica, de baixo a médio se a origem é costeira, e médio para alto se a origem é ribeirinha. Aguirre-Macedo et al. (2008) observaram que a água de lastro proveniente de rios tem maiores concentrações de coliformes do que aquelas 
provenientes da costa ou do oceano, em um estudo relacionado a um recife de corais. Tal escala de risco pode ser um ponto de partida na especificação de uma distribuição a priori. Chu et al. (1997) observaram que o número de espécies de organismos diminui com a "idade" da água de lastro em um estudo em Hong Kong. Também observaram que algumas espécies foram capazes de sobreviver por mais de um ano. Considere "idade" da água de lastro o tempo contado desde o momento em que a água foi armazenada no navio. Faulkner et al. (2014) propuseram um procedimento interessante para desenvolver uma lista de monitoramento de espécies de organismos invasivos para uma região escolhida. Os passos para construção da lista são, grosso modo, os seguintes: (1) obtenha uma lista global de espécies invasivas, (2) filtre as espécies já presentes na região escolhida (espécies nativas ou que já foram introduzidas), (3) filtre as espécies que não são ambientalmente propícias de se desenvolverem na região escolhida, e em seguida verifique se há possibilidade dessas espécies se propagarem desde sua região nativa até a região escolhida. Para mais detalhes, vide Faulkner et al. (2014). Outra informação importante que pode ser considerada é se houve algum tipo de gerenciamento ou tratamento da água de lastro do navio, como por exemplo troca oceânica, filtração, radiação ultravioleta etc., técnicas que visam a redução da concentração de organismos (vide e.g. Balaji et al., 2014; Tsolaki \& Diamadopoulos, 2010). Trabalhos que apresentam metodologias que podem ser usadas para classificar o risco de introdução de organismos de acordo com a região climática, salinidade, distancia da viagem etc., são Gollasch \& Leppäkoski (2007), Liu et al. (2014), Drake \& Mandrak (2010, 2014), entre outros. Todos esses aspectos podem servir como componentes na especificação de uma distribuição a priori, na qual deixamos para pesquisas futuras.

\subsection{Considerações finais}

Apresentamos uma abordagem bayesiana como alternativa à abordagem frequentista para a determinação do tamanho amostral. Destacamos o uso do processo Dirichlet em conjunto com a distribuição Poisson. Outro passo é acoplar as metodologias desenvolvidas aqui em sistemas automáticos de monitoramento por imagem como o desenvolvido por Matuszewski et al. (2015), que podem permitir um procedimento sequencial ao longo do deslastre.

Apesar desta tese ser focada no problema da água de lastro, as metodologias desenvolvidas aqui podem ser amplamente utilizadas em outros problemas. Todos os cálculos e algoritmos foram programados em linguagem R (R Core Team, 2016) e podem ser acessados em www.github.com/ eliardocosta. 
Tabela 4.11: Número mínimo de alíquotas ( $n$ ) com informação a priori $\lambda_{0}=10(w / \phi)$ sob o modelo (2.3)-(2.4) com a função perda 2.

\begin{tabular}{|c|c|c|c|c|c|c|c|}
\hline \multirow{2}{*}{$\begin{array}{l}\text { Volume da } \\
\text { alíquota }(w)\end{array}$} & \multirow{2}{*}{$\begin{array}{l}\text { Custo de uma } \\
\text { alíquota }(c)\end{array}$} & \multirow[b]{2}{*}{$\gamma$} & \multirow{2}{*}{$\phi$} & \multicolumn{4}{|c|}{ Parâmetro de forma $\left(\theta_{0}\right)$} \\
\hline & & & & 11 & 25 & 50 & 75 \\
\hline \multirow{20}{*}{0,5} & \multirow{10}{*}{0,005} & \multirow{5}{*}{1} & 1,0 & 205 & 193 & 175 & 158 \\
\hline & & & 2,5 & 202 & 191 & 173 & 157 \\
\hline & & & 5,0 & 201 & 190 & 172 & 156 \\
\hline & & & 7,5 & 201 & 190 & 172 & 156 \\
\hline & & & 10,0 & 201 & 189 & 172 & 156 \\
\hline & & \multirow{5}{*}{$1 / 4$} & 1,0 & 128 & 118 & 104 & 93 \\
\hline & & & 2,5 & 126 & 117 & 103 & 92 \\
\hline & & & 5,0 & 126 & 116 & 102 & 92 \\
\hline & & & 7,5 & 125 & 116 & 102 & 91 \\
\hline & & & 10,0 & 125 & 116 & 102 & 91 \\
\hline & \multirow{10}{*}{0,010} & \multirow{5}{*}{1} & 1,0 & 129 & 119 & 105 & 93 \\
\hline & & & 2,5 & 126 & 117 & 103 & 92 \\
\hline & & & 5,0 & 125 & 116 & 102 & 92 \\
\hline & & & 7,5 & 125 & 116 & 102 & 91 \\
\hline & & & 10,0 & 125 & 116 & 102 & 91 \\
\hline & & \multirow{5}{*}{$1 / 4$} & 1,0 & 81 & 73 & 63 & 55 \\
\hline & & & 2,5 & 79 & 71 & 61 & 54 \\
\hline & & & 5,0 & 78 & 71 & 61 & 54 \\
\hline & & & 7,5 & 78 & 71 & 61 & 54 \\
\hline & & & 10,0 & 78 & 71 & 61 & 53 \\
\hline \multirow{20}{*}{1,0} & \multirow{10}{*}{0,005} & \multirow{5}{*}{1} & 1,0 & 167 & 161 & 151 & 143 \\
\hline & & & 2,5 & 164 & 154 & 149 & 141 \\
\hline & & & 5,0 & 163 & 158 & 149 & 140 \\
\hline & & & 7,5 & 163 & 158 & 148 & 140 \\
\hline & & & 10,0 & 163 & 157 & 148 & 140 \\
\hline & & \multirow{5}{*}{$1 / 4$} & 1,0 & 106 & 101 & 98 & 87 \\
\hline & & & 2,5 & 104 & 99 & 92 & 85 \\
\hline & & & 5.0 & 103 & 98 & 91 & 84 \\
\hline & & & 7,5 & 103 & 98 & 91 & 84 \\
\hline & & & 10,0 & 103 & 98 & 91 & 84 \\
\hline & \multirow{10}{*}{0,010} & \multirow{5}{*}{1} & 1,0 & 107 & 101 & 93 & 86 \\
\hline & & & 2,5 & 104 & 99 & 91 & 85 \\
\hline & & & 5.0 & 103 & 98 & 91 & 84 \\
\hline & & & 7,5 & 103 & 98 & 91 & 84 \\
\hline & & & 10,0 & 103 & 98 & 90 & 84 \\
\hline & & \multirow{5}{*}{$1 / 4$} & 1,0 & 67 & 63 & 57 & 52 \\
\hline & & & 2,5 & 66 & 62 & 56 & 51 \\
\hline & & & 5,0 & 65 & 61 & 55 & 51 \\
\hline & & & 7,5 & 65 & 61 & 55 & 51 \\
\hline & & & 10,0 & 65 & 61 & 55 & 51 \\
\hline
\end{tabular}


Tabela 4.12: Número mínimo de alíquotas ( $n$ ) com informação a priori $\lambda_{0}=10$ sob o modelo (2.5)-(2.7) com a função perda 3 e $W(d t)$ sendo a função densidade de uma distribuição normal com média e desvio padrão iguais a 10.

\begin{tabular}{|c|c|c|c|c|c|c|c|}
\hline \multirow{2}{*}{$\begin{array}{l}\text { Volume da } \\
\text { alíquota }(w)\end{array}$} & \multirow{2}{*}{$\begin{array}{l}\text { Custo de uma } \\
\text { alíquota }(c)\end{array}$} & \multirow{2}{*}{$\alpha^{*}$} & \multicolumn{5}{|c|}{ Parâmetro de forma $\left(\theta_{0}\right)$} \\
\hline & & & 1,0 & 2,5 & 5,0 & 7,5 & 10,0 \\
\hline \multirow{10}{*}{0,5} & \multirow{5}{*}{0,005} & 0,5 & 18 & 14 & 12 & 10 & 9 \\
\hline & & 1,5 & 19 & 16 & 14 & 12 & 11 \\
\hline & & 2,5 & 18 & 16 & 14 & 12 & 12 \\
\hline & & 5,0 & 16 & 14 & 12 & 11 & 10 \\
\hline & & 10,0 & 12 & 11 & 9 & 8 & 8 \\
\hline & \multirow{5}{*}{0,010} & 0,5 & 12 & 10 & 8 & 7 & 6 \\
\hline & & 1,5 & 13 & 11 & 9 & 8 & 8 \\
\hline & & 2,5 & 12 & 11 & 9 & 8 & 8 \\
\hline & & 5,0 & 11 & 9 & 8 & 7 & 7 \\
\hline & & 10,0 & 8 & 7 & 6 & 5 & 5 \\
\hline \multirow{10}{*}{1,0} & \multirow{5}{*}{0,005} & 0,5 & 19 & 16 & 13 & 11 & 10 \\
\hline & & 1,5 & 19 & 16 & 14 & 13 & 12 \\
\hline & & 2,5 & 18 & 16 & 14 & 12 & 12 \\
\hline & & 5,0 & 16 & 14 & 12 & 11 & 10 \\
\hline & & 10,0 & 12 & 11 & 9 & 8 & 8 \\
\hline & \multirow{5}{*}{0,010} & 0,5 & 13 & 11 & 8 & 7 & 6 \\
\hline & & 1,5 & 13 & 11 & 9 & 8 & 8 \\
\hline & & 2,5 & 12 & 11 & 9 & 8 & 8 \\
\hline & & 5,0 & 10 & 9 & 8 & 7 & 7 \\
\hline & & 10,0 & 8 & 7 & 6 & 5 & 5 \\
\hline
\end{tabular}




\section{Apêndice A}

\section{Modelo Poisson com distribuição a}

\section{priori gama}

\section{A.1 Distribuição a posteriori e propriedades}

Sabemos que sob o modelo (2.1)-(2.2), cada $X_{i}$ seguirá marginalmente uma distribuição binomial negativa com média $w \lambda_{0}$ e parâmetro $\theta_{0}$, i.e., $X_{i} \sim B N\left(w \lambda_{0}, \theta_{0}\right)$. Além disso, $S_{n}=\sum_{i=1}^{n} X_{i} \sim$ $B N\left(n w \lambda_{0}, n \theta_{0}\right)$.

A função verossimilhança correspondente ao modelo (2.1) é

$$
f\left(\lambda ; \boldsymbol{x}_{n}\right)=\prod_{i=1}^{n} \frac{e^{-w \lambda}(w \lambda)^{x_{i}}}{x_{i} !}=\frac{e^{-n w \lambda}(w \lambda)^{s_{n}}}{\prod_{i=1}^{n} x_{i} !}
$$

em que $s_{n}=\sum_{i=1}^{n} x_{i}$ e $\boldsymbol{x}_{n}=\left(x_{1}, \ldots, x_{n}\right)$. Considerando uma distribuição a priori gama (2.2) para $\lambda$, a distribuição a posteriori é

$$
F\left(d \lambda \mid \boldsymbol{x}_{n}\right) \propto \lambda^{s_{n}} \exp (-n w \lambda) \times \lambda^{\theta_{0}-1} \exp \left(-\frac{\theta_{0}}{\lambda_{0}} \lambda\right)=\lambda^{\theta_{0}+s-1} \exp \left[-\left(n w+\frac{\theta_{0}}{\lambda_{0}}\right) \lambda\right]
$$

que é uma distribuição gama com parâmetros $\theta_{0}+s_{n}$ e $n w+\theta_{0} / \lambda_{0}$. A média e a variância dessa distribuição a posteriori são, respectivamente,

$$
\mathbb{E}\left[\lambda \mid \boldsymbol{x}_{n}\right]=\frac{\theta_{0}+s_{n}}{\theta_{0} / \lambda_{0}+n w} \quad \text { e } \quad \operatorname{Var}\left[\lambda \mid \boldsymbol{x}_{n}\right]=\frac{\theta_{0}+s_{n}}{\left(\theta_{0} / \lambda_{0}+n w\right)^{2}}
$$

Para determinar o intervalo HPD de tamanho $\ell$ para uma distribuição gama com parâmetros $\kappa$ e $\psi$, denotada por $G(\kappa, \psi)$, é necessário resolver a equação $g(a)=g(a+\ell)$ em $a$, em que $g(\cdot)$ é a 
correspondente função densidade (Stamey et al., 2006). A solução é

$$
a=\frac{\ell}{\exp (\psi \ell /(\kappa-1))-1} .
$$

Para a distribuição a posteriori resultante do modelo Poisson com distribuição a priori gama que consideramos temos $\kappa=\theta_{0}+s_{n}$ e $\psi=n w+\theta_{0} / \lambda_{0}$.

\section{A.2 Demonstração do Teorema 3.1}

Antes de apresentar a demonstração do Teorema 3.1 precisamos de alguns resultados.

Lema A.1. Seja $\left\{X_{n}\right\}_{n \geq 1}$ uma sequência de variáveis aleatórias. Se $\sup _{n} \mathbb{E}\left[\left|X_{n}\right|^{1+\epsilon}\right]<\infty$ para algum $\epsilon>0$, então $X_{n}$ é uniformemente integrável.

Demonstração. Vide Billingsley (1995, p. 338).

Teorema A.1. Se $X_{n} \rightarrow X$ em distribuição quando $n \rightarrow \infty$ e $X_{n}$ é uniformemente integrável, então $X$ é integrável e $\mathbb{E}\left[X_{n}\right] \rightarrow \mathbb{E}[X]$, quando $n \rightarrow \infty$.

Demonstração. Vide Billingsley (1995, Teorema 25.12).

Teorema A.2. Suponha que $X_{n} \rightarrow X$ em distribuição quando $n \rightarrow \infty$ e que $h_{n}(x)$ e $h(x)$ são funções Borel-mensuráveis. Seja $E \subseteq \mathbb{R}$ um conjunto em que, para alguma sequência $x_{n} \rightarrow x$, a propriedade $h_{n}(x) \rightarrow h(x)$ quando $n \rightarrow \infty$ não vale. Suponha que $\mathbb{P}[X(\omega) \in E]=0$. Então, $h_{n}\left(X_{n}\right) \rightarrow h(X)$ em distribuição quando $n \rightarrow \infty$.

Demonstração. Vide Billingsley (1995, p. 340).

Considerando o critério do comprimento médio temos que $\ell^{\prime}\left(\boldsymbol{x}_{n}, n\right)$ pode ser aproximado por $2 z_{\rho / 2} \sqrt{\operatorname{Var}\left[\lambda \mid \boldsymbol{x}_{n}\right]}$, em que $\operatorname{Var}\left[\lambda \mid \boldsymbol{x}_{n}\right]$ é dada em (A.2) e $z_{\rho / 2}$ é o quantil de probabilidade $1-\rho / 2$ da distribuição normal padrão, uma vez que a variância a posteriori pode estar relacionada indiretamente ao tamanho do correspondente intervalo HPD (Joseph et al., 1995).

Proposição A.1. Considere o modelo (2.1)-(2.2) e defina $S_{n}=\sum_{i=1}^{n} X_{i}$. Então,

$$
\lim _{n \rightarrow \infty}\left(n w+\frac{\theta_{0}}{\lambda_{0}}\right)^{1 / 2} \frac{\mathbb{E}\left[\ell^{\prime}\left(\boldsymbol{X}_{n}, n\right)\right]}{2 z_{\rho / 2}}=\frac{\Gamma\left(\theta_{0}+1 / 2\right)}{\Gamma\left(\theta_{0}\right)}\left(\frac{\lambda_{0}}{\theta_{0}}\right)^{1 / 2} .
$$

Demonstração. Sejam $Y_{n}=S_{n} / n w$ e $\mathcal{F}$ o conjunto de pontos em que $Y_{n}$ tem probabilidade positiva. Pelo Teorema B.1 de M'Lan et al. (2006), temos que $S_{n} / n-w \lambda \rightarrow 0$ em probabilidade quando $n \rightarrow \infty$. Logo, $Y_{n}-\lambda \rightarrow 0$ em distribuição quando $n \rightarrow \infty$ (vide Sen et al., 2009, Teorema 6.2.7). 
Seja $h_{n}(y)=\left[\frac{\theta_{0}+n w y}{n w+\theta_{0} / \lambda_{0}}\right]^{1 / 2}$ e $h(y)=y^{1 / 2}=\lim _{n \rightarrow \infty} h_{n}(y)$. Considere o Lema A.1 com $\epsilon=1$; então,

$$
\begin{aligned}
\mathbb{E}\left[\left|Y_{n}\right|^{1+\epsilon}\right] & =\frac{\mathbb{E}\left[\left|S_{n}\right|^{1+\epsilon}\right]}{(n w)^{1+\epsilon}}=\frac{\mathbb{E}\left[\left|S_{n}\right|^{2}\right]}{(n w)^{2}}=\frac{1}{(n w)^{2}}\left[\operatorname{Var}\left[S_{n}\right]+\left(\mathbb{E}\left[S_{n}\right]\right)^{2}\right] \\
& =\frac{1}{(n w)^{2}}\left[n w \lambda_{0}+\frac{n\left(w \lambda_{0}\right)^{2}}{\theta_{0}}+\left(n w \lambda_{0}\right)^{2}\right]=\frac{\lambda_{0}}{n w}+\lambda_{0}^{2}\left(1+\frac{1}{n \theta_{0}}\right)
\end{aligned}
$$

Logo,

$$
\sup _{n} \mathbb{E}\left[\left|Y_{n}\right|^{1+\epsilon}\right]=\sup _{n}\left[\frac{\lambda_{0}}{n w}+\lambda_{0}^{2}\left(1+\frac{1}{n \theta_{0}}\right)\right]=\frac{\lambda_{0}}{w}+\lambda_{0}^{2}\left(1+\frac{1}{\theta_{0}}\right)<\infty
$$

e pelo Lema A.1, $Y_{n}$ é uniformemente integrável. Pelo Teorema A.1, temos $\mathbb{E}\left[Y_{n}\right] \rightarrow \mathbb{E}[\lambda]$ quando $n \rightarrow \infty$ e pelo Teorema A.2, $h_{n}\left(Y_{n}\right) \rightarrow h(\lambda)$ em distribuição quando $n \rightarrow \infty$. Pelos resultados anteriores, temos

$$
\begin{aligned}
\left(n w+\frac{\theta_{0}}{\lambda_{0}}\right)^{1 / 2} \frac{\mathbb{E}\left[\ell^{\prime}\left(\boldsymbol{X}_{n}, n\right)\right]}{2 z_{\rho / 2}} & \cong \sum_{s_{n}=0}^{\infty}\left[\frac{\theta_{0}+s_{n}}{n w+\theta_{0} / \lambda_{0}}\right]^{1 / 2} f_{S_{n}}\left(s_{n}\right)=\sum_{y_{n} \in \mathcal{F}} h_{n}\left(y_{n}\right) f_{Y_{n}}\left(y_{n}\right) \\
& =\mathbb{E}\left[h\left(Y_{n}\right)\right] \rightarrow \mathbb{E}[h(\lambda)] \\
& =\int_{0}^{\infty} h(\lambda) \frac{\left(\theta_{0} / \lambda_{0}\right)^{\theta_{0}}}{\Gamma\left(\theta_{0}\right)} \lambda^{\theta_{0}-1} \exp \left(-\theta_{0} \lambda / \lambda_{0}\right) d \lambda \\
& =\int_{0}^{\infty} \frac{\left(\theta_{0} / \lambda_{0}\right)^{\theta_{0}}}{\Gamma\left(\theta_{0}\right)} \lambda^{\theta_{0}+1 / 2-1} \exp \left(-\theta_{0} \lambda / \lambda_{0}\right) d \lambda \\
& =\left(\frac{\lambda_{0}}{\theta_{0}}\right)^{1 / 2} \frac{\Gamma\left(\theta_{0}+1 / 2\right)}{\Gamma\left(\theta_{0}\right)} .
\end{aligned}
$$

Finalmente, consideremos a demonstração do Teorema 3.1. Pela Proposição A.1, o critério do comprimento médio pode ser aproximado usando (A.4). Logo, basta obter o menor valor de $n$ que satisfaça

$$
\frac{2 z_{\rho / 2}}{\left(n w+\theta_{0} / \lambda_{0}\right)^{1 / 2}} \frac{\Gamma\left(\theta_{0}+1 / 2\right)}{\Gamma\left(\theta_{0}\right)}\left(\frac{\lambda_{0}}{\theta_{0}}\right)^{1 / 2} \leq \ell_{\max },
$$

e resolvendo a inequação em $n$ obtemos o resultado do referido teorema. 


\section{A.3 Obtenção da decisão de Bayes usando MCT}

\section{A.3.1 Função perda 1}

Obter a decisão $d_{n}^{*}$ que minimiza o risco de Bayes a posteriori é equivalente a obter aquela que minimiza o valor esperado a posteriori da função perda. Considerando a função perda 1, temos

$$
\begin{aligned}
\mathbb{E}\left[L\left(\lambda, d_{n}\right) \mid \boldsymbol{x}_{n}\right] & =\rho \tau+\int_{0}^{\infty}(a-\lambda)^{+} F\left(d \lambda \mid \boldsymbol{x}_{n}\right)+\int_{0}^{\infty}(\lambda-b)^{+} F\left(d \lambda \mid \boldsymbol{x}_{n}\right) \\
& =\int_{b}^{\infty} \lambda F\left(d \lambda \mid \boldsymbol{x}_{n}\right)-\int_{0}^{a} \lambda F\left(d \lambda \mid \boldsymbol{x}_{n}\right) \\
& +\frac{\rho(b-a)}{2}+a \int_{0}^{a} F\left(d \lambda \mid \boldsymbol{x}_{n}\right)-b \int_{b}^{\infty} F\left(d \lambda \mid \boldsymbol{x}_{n}\right) .
\end{aligned}
$$

Note que se $a$ e $b$ forem os quantis de probabilidades $\rho / 2$ e $1-\rho / 2$ da distribuição a posteriori, respectivamente, a soma dos três últimos termos da expressão (A.5) será igual a zero, indicando que o mínimo foi atingido (Rice et al., 2008). Então,

$$
\mathbb{E}\left[L\left(\lambda, d_{n}^{*}\right) \mid \boldsymbol{x}_{n}\right]=\int_{b}^{\infty} \lambda F\left(d \lambda \mid \boldsymbol{x}_{n}\right)-\int_{0}^{a} \lambda F\left(d \lambda \mid \boldsymbol{x}_{n}\right)
$$

Considerando o modelo (2.1)-(2.2), a distribuição a posteriori será gama com parâmetros $\kappa=\theta_{0}+s_{n}$ e $\psi=n w+\theta_{0} / \lambda_{0} ; \operatorname{logo}$,

$$
\int_{0}^{a} \lambda F\left(d \lambda \mid \boldsymbol{x}_{n}\right)=\int_{0}^{a} \lambda \frac{\psi^{\kappa}}{\Gamma(\kappa)} \lambda^{\kappa-1} \exp (-\psi \lambda) d \lambda=\frac{\kappa}{\psi} \int_{0}^{a} \frac{\psi^{\kappa+1}}{\Gamma(\kappa+1)} \lambda^{\kappa} \exp (-\psi \lambda) d \lambda .
$$

Note que a última integral é a probabilidade acumulada até o ponto $a$ de uma distribuição gama com parâmetros $\kappa+1$ e $\psi$; de modo análogo, obtemos a outra integral de $\mathbb{E}\left[L\left(\lambda, d_{n}^{*}\right) \mid \boldsymbol{x}_{n}\right]$ e podemos reescrever esse valor esperado condicional como

$$
\mathbb{E}\left[L\left(\lambda, d_{n}^{*}\right) \mid \boldsymbol{x}_{n}\right]=\frac{\kappa}{\psi}\left[\int_{b}^{\infty} \frac{\psi^{\kappa+1}}{\Gamma(\kappa+1)} \lambda^{\kappa} \exp (-\psi \lambda) d \lambda-\int_{0}^{a} \frac{\psi^{\kappa+1}}{\Gamma(\kappa+1)} \lambda^{\kappa} \exp (-\psi \lambda) d \lambda\right]
$$

\section{A.3.2 Função perda 2}

Considerando a função perda 2, o valor esperado da perda a posteriori é

$$
\mathbb{E}\left[L\left(\lambda, d_{n}\right) \mid \boldsymbol{x}_{n}\right]=\gamma \tau+\int_{0}^{\infty} \frac{(\lambda-m)^{2}}{\tau} F\left(d \lambda \mid \boldsymbol{x}_{n}\right)
$$


A integral do segundo membro será minimizada se $m=\mathbb{E}\left[\lambda \mid \boldsymbol{x}_{n}\right]$; logo,

$$
\mathbb{E}\left[L\left(\lambda, d_{n}\right) \mid \boldsymbol{x}_{n}\right]=\gamma \tau+\frac{\operatorname{Var}\left[\lambda \mid \boldsymbol{x}_{n}\right]}{\tau} .
$$

Resta agora minimizar o valor esperado a posteriori em relação a $\tau$; considerando $\tau=$ $t f(\gamma) \sqrt{\operatorname{Var}\left[\lambda \mid \boldsymbol{x}_{n}\right]}$, para $t>0$ e $f(\gamma)$ uma função positiva qualquer de $\gamma$ (Rice et al., 2008), temos

$$
\mathbb{E}\left[L\left(\lambda, d_{n}\right) \mid \boldsymbol{x}_{n}\right]=\sqrt{\operatorname{Var}\left[\lambda \mid \boldsymbol{x}_{n}\right]}\left[t f(\gamma) \gamma+\frac{1}{t f(\gamma)}\right] .
$$

Derivando a expressão entre colchetes em relação a $t$ obtemos o seu mínimo quando $t=1 /\left[\gamma^{1 / 2} f(\gamma)\right]$, substituindo este valor na expressão de $\tau$ obtemos

$$
\tau=t f(\gamma) \sqrt{\operatorname{Var}\left[\lambda \mid \boldsymbol{x}_{n}\right]}=\frac{1}{\gamma^{1 / 2} f(\gamma)} f(\gamma) \sqrt{\operatorname{Var}\left[\lambda \mid \boldsymbol{x}_{n}\right]}=\gamma^{-1 / 2} \sqrt{\operatorname{Var}\left[\lambda \mid \boldsymbol{x}_{n}\right]} .
$$

Outra maneira de obter o mínimo é derivar (A.8) diretamente em relação a $\tau$ e igualando o resultado a zero. Logo, a decisão de Bayes é o intervalo $[a, b]=\left[m-\mathrm{SD}_{\gamma}, m+\mathrm{SD}_{\gamma}\right]$, em que $\left(m, \mathrm{SD}_{\gamma}\right)=\left(\mathbb{E}\left[\lambda \mid \boldsymbol{x}_{n}\right], \gamma^{-1 / 2} \sqrt{\operatorname{Var}\left[\lambda \mid \boldsymbol{x}_{n}\right]}\right)$. Dessa forma, o valor esperado da perda a posteriori será

$$
\mathbb{E}\left[L\left(\lambda, d_{n}^{*}\right) \mid \boldsymbol{x}_{n}\right]=\gamma \gamma^{-1 / 2} \sqrt{\operatorname{Var}\left[\lambda \mid \boldsymbol{x}_{n}\right]}+\frac{\operatorname{Var}\left[\lambda \mid \boldsymbol{x}_{n}\right]}{\gamma^{-1 / 2} \sqrt{\operatorname{Var}\left[\lambda \mid \boldsymbol{x}_{n}\right]}}=2 \gamma^{1 / 2} \sqrt{\operatorname{Var}\left[\lambda \mid \boldsymbol{x}_{n}\right]}
$$

Dados o modelo (2.1)-(2.2) e (A.2), podemos facilmente calcular $\mathbb{E}\left[L\left(\lambda, d_{n}^{*}\right) \mid \boldsymbol{x}_{n}\right]$.

\section{A.4 Algoritmos necessários para o cálculo do tamanho amostral}

\section{A.4.1 CCobM}

Um possível algoritmo para obtenção do tamanho da amostra $n$ usando o CCobM é descrito na sequência.

Passo 1. Fixe $n=1, \ell, \lambda_{0}, \theta_{0}, w$ e $\rho$;

Passo 2. Simule uma amostra de tamanho $M(e . g ., M=1000)$ de $s_{n}$ de uma distribuição binomial negativa com média $n w \lambda_{0}$ e parâmetro de dispersão $n \theta_{0}$; o tamanho da amostra simulada $M$ deve ser tão grande quanto possível a fim de melhorar a estimativa da integral (ou valor esperado) referente ao critério;

Passo 3. Calcule o limitante inferior $a$ do intervalo HPD usando (A.3) e a probabilidade a posteriori 
$\mathbb{P}\left[a \leq \lambda \leq a+\ell \mid s_{n}\right]$ usando (A.1) para cada $s_{n}$ simulado; para cada valor simulado no Passo 2 calcule $\theta_{0}+s_{n}$ e $n w+\theta_{0} / \lambda_{0}$ referentes à distribuição a posteriori gama de $\lambda$, e então calcule o limitante inferior $a$ do intervalo HPD usando (A.3). Em seguida, calcule as probabilidades a posteriori usando a distribuição a posteriori gama com parâmetros $\theta_{0}+s_{n}$ e $n w+\theta_{0} / \lambda_{0}(\mathrm{~A} .1)$;

Passo 4. Calcule a média das $M$ probabilidades a posteriori;

Passo 5. Se essa média for $\geq 1-\rho$, pare. O valor $n$ obtido nesse passo é o valor desejado. Caso contrário, faça $n=n+1$ e volte ao Passo 2 .

\section{A.4.2 CCompM}

Um possível algoritmo para obtenção do tamanho da amostra $n$ usando o CCompM é descrito abaixo.

Passo 1. Fixe $n=1, \ell_{\max }, \lambda_{0}, \theta_{0}, w$ e $\rho$;

Passo 2. Simule uma amostra de tamanho $M($ e.g., $M=1000)$ de $s_{n}$ de uma distribuição binomial negativa com média $n w \lambda_{0}$ e parâmetro de dispersão $n \theta_{0}$;

Passo 3. Calcule o comprimento do intervalo de credibilidade a posteriori $(1-\rho)$ para cada $s_{n}$ simulado; para cada valor simulado no Passo 2 calcule $\theta_{0}+s_{n}$ e $n w+\theta_{0} / \lambda_{0}$ referentes à distribuição a posteriori gama de $\lambda$, e então obtenha os quantis de ordens $\rho / 2$ e $1-\rho / 2$. Em seguida, calcule a diferença entre os quantis para cada valor simulado a fim de obter o comprimento do intervalo de credibilidade;

Passo 4. Calcule a média dos $M$ comprimentos dos intervalos de credibilidade a posteriori;

Passo 5. Se essa média for $\leq \ell_{\max }$, pare. O valor $n$ fixado nesse passo é o valor desejado. Caso contrário, faça $n=n+1$ e volte ao Passo 2 .

\section{A.4.3 MCT}

A escolha do conjunto na qual $n$ irá variar durante a execução do algoritmo é arbitrária, dessa forma consideramos $n=1,5,10, \ldots, 95,100$ e para cada valor de $n$ nesse conjunto a estimativa de $C T(n)$ foi calculada 10 (número arbitrário também) vezes, i.e., obtemos 10 estimativas para $C T(n)$. Um possível algoritmo para obtenção do tamanho da amostra $n$ usando o MCT com a função perda 1 ou a 2 é descrito como segue. 
Passo 1. Fixe $\lambda_{0}, \theta_{0}, w, c$, e $\rho$ (função perda 1) ou $\gamma$ (função perda 2), além de um conjunto na qual $n$ irá variar;

Passo 2. Para cada $n$ simule uma amostra de tamanho $M($ e.g., $M=1000)$ de $s_{n}$ de uma distribuição binomial negativa com média $n w \lambda_{0}$ e parâmetro de dispersão $n \theta_{0}$, calcule os respectivos limitantes $a$ e $b$ (no caso da função perda 1 ), em seguida os respectivos $\mathbb{E}\left[L\left(\lambda, d_{n}^{*}\right) \mid \boldsymbol{x}_{n}\right]$ usando (A.7) ou (A.9), e finalmente calcule a média desses $M$ valores esperados. Esse valor será a estimativa do risco de Bayes a posteriori minimizado para o respectivo $n$;

Passo 3. Para cada risco de Bayes a posteriori estimado, some o respectivo valor de custo cn, guarde esses valores;

Passo 4. Com os valores obtidos no Passo 3 e os respectivos valores de $n$ ajuste o modelo de regressão (3.5) e obtenha o valor mínimo de $n$ usando (3.6). 
APÊNDICE A 


\section{Apêndice B}

\section{Modelo binomial negativo com}

\section{distribuição a priori Pearson Tipo VI}

\section{B.1 Distribuição a posteriori e propriedades}

Considere o modelo (2.3). A correspondente função verossimilhança é dada por

$$
\begin{aligned}
f\left(\lambda ; \boldsymbol{x}_{n}\right) & =\prod_{i=1}^{n} \frac{\Gamma\left(\phi+x_{i}\right)}{\Gamma\left(x_{i}+1\right) \Gamma(\phi)}\left(\frac{w \lambda}{w \lambda+\phi}\right)^{x_{i}}\left(\frac{\phi}{w \lambda+\phi}\right)^{\phi} \\
& =\left[\prod_{i=1}^{n} \frac{\Gamma\left(\phi+x_{i}\right)}{\Gamma\left(x_{i}+1\right) \Gamma(\phi)}\right]\left(\frac{w}{\phi} \lambda\right)^{s_{n}}\left(1+\frac{w}{\phi} \lambda\right)^{-s_{n}-n \phi}
\end{aligned}
$$

em que $s_{n}=\sum_{i=1}^{n} x_{i}$ e $\boldsymbol{x}_{n}=\left(x_{1}, \ldots, x_{n}\right)$. Considerando uma distribuição a priori Pearson Tipo VI (2.4) para $\lambda$, a distribuição a posteriori é

$$
\begin{aligned}
F\left(d \lambda \mid \boldsymbol{x}_{n}\right) & \propto\left(\frac{w}{\phi} \lambda\right)^{s_{n}}\left(1+\frac{w}{\phi} \lambda\right)^{-s_{n}-n \phi} \times\left(\frac{w}{\phi} \lambda\right)^{\theta_{0}-1}\left(1+\frac{w}{\phi} \lambda\right)^{-\theta_{0}-\left(\theta_{0} / \lambda_{0}+1\right)} \\
& =\left(\frac{w}{\phi} \lambda\right)^{\theta_{0}+s_{n}-1}\left(1+\frac{w}{\phi} \lambda\right)^{-\left(\theta_{0}+s_{n}\right)-\left(\theta_{0} / \lambda_{0}+n \phi+1\right)}
\end{aligned}
$$

que corresponde a uma distribuição Pearson Tipo VI com parâmetros de localização e escala iguais a 0 e $\phi / w$ e parâmetros de forma $\theta_{0}+s_{n}$ e $\theta_{0} / \lambda_{0}+n \phi+1$. A média e a variância dessa distribuição a posteriori são dadas, respectivamente, por

$$
\mathbb{E}\left[\lambda \mid \boldsymbol{x}_{n}\right]=\frac{\phi}{w} \frac{\theta_{0}+s_{n}}{\theta_{0} / \lambda_{0}+n \phi} \quad \text { e } \quad \operatorname{Var}\left[\lambda \mid \boldsymbol{x}_{n}\right]=\left(\frac{\phi}{w}\right)^{2} \frac{\theta_{0}+s_{n}}{\left(\theta_{0} / \lambda_{0}+n \phi\right)^{2}}\left(\frac{\mathbb{E}\left[\lambda \mid \boldsymbol{x}_{n}\right]+1}{1-q}\right)
$$

em que $q=\left(\theta_{0} / \lambda_{0}+n \phi\right)^{-1}$. Para determinar o intervalo HPD de tamanho $\ell$ para uma distribuição 
Pearson Tipo VI, denotada por $P V I(0, \phi / w, \kappa, \psi)$, é necessário resolver a equação $g(a)=g(a+\ell)$ em $a$, em que $g(\cdot)$ é a correspondente função densidade. A solução é obtida da equação

$$
(\kappa-1) \log \left[1+\frac{\ell}{a}\right]-(\kappa+\psi) \log \left[1+\frac{w \ell}{\phi+w a}\right]=0
$$

que pode ser revolvida por meio de métodos numéricos (e.g., Newton-Raphson). Para a distribuição a posteriori resultante do modelo binomial negativo com distribuição a priori Pearson Tipo VI, temos $\kappa=\theta_{0}+s_{n}$ e $\psi=\theta_{0} / \lambda_{0}+n \phi+1$.

\section{B.2 Obtenção da decisão de Bayes usando MCT}

\section{B.2.1 Função perda 1}

Considerando a função perda 1 e de acordo com (A.6), temos que

$$
\mathbb{E}\left[L\left(\lambda, d_{n}^{*}\right) \mid \boldsymbol{x}_{n}\right]=\int_{b}^{\infty} \lambda F\left(d \lambda \mid \boldsymbol{x}_{n}\right)-\int_{0}^{a} \lambda F\left(d \lambda \mid \boldsymbol{x}_{n}\right),
$$

em que $a$ e $b$ são os quantis de probabilidades $\rho / 2$ e $1-\rho / 2$ da distribuição a posteriori, respectivamente. Dado o modelo (2.3)-(2.4), a distribuição a posteriori será $P V I(0, \phi / w, \kappa, \psi)$, em que $\kappa=\theta_{0}+s_{n}$ e $\psi=\theta_{0} / \lambda_{0}+n \phi+1 ; \log \mathrm{O}$

$$
\int_{0}^{a} \lambda F\left(d \lambda \mid \boldsymbol{x}_{n}\right)=\int_{0}^{a} \lambda\left(\frac{w}{\phi} \lambda\right)^{\kappa-1}\left(1+\frac{w}{\phi} \lambda\right)^{-\kappa-\psi} d \lambda=\frac{\phi}{w} \int_{0}^{a}\left(\frac{w}{\phi} \lambda\right)^{\kappa}\left(1+\frac{w}{\phi} \lambda\right)^{-(\kappa+1)-(\psi-1)} d \lambda
$$

Note que a última integral é a probabilidade acumulada até o ponto $a$ de uma distribuição $P V I(0, \phi / w, \kappa+1, \psi-1)$. De modo análogo obtemos a outra integral de $\mathbb{E}\left[L\left(\lambda, d_{n}^{*}\right) \mid \boldsymbol{x}_{n}\right]$ e podemos reescrever esse valor esperado condicional da seguinte forma:

$$
\begin{aligned}
\mathbb{E}\left[L\left(\lambda, d_{n}^{*}\right) \mid \boldsymbol{x}_{n}\right] & =\frac{\phi}{w}\left[\int_{b}^{\infty}\left(\frac{w}{\phi} \lambda\right)^{\kappa}\left(1+\frac{w}{\phi} \lambda\right)^{-(\kappa+1)-(\psi-1)} d \lambda\right] \\
& -\frac{\phi}{w}\left[\int_{0}^{a}\left(\frac{w}{\phi} \lambda\right)^{\kappa}\left(1+\frac{w}{\phi} \lambda\right)^{-(\kappa+1)-(\psi-1)} d \lambda\right]
\end{aligned}
$$

\section{B.2.2 Função perda 2}

Considerando a função perda 2 e de acordo com (A.9), temos que

$$
\mathbb{E}\left[L\left(\lambda, d_{n}^{*}\right) \mid \boldsymbol{x}_{n}\right]=2 \gamma^{1 / 2} \sqrt{\mathbb{V}\left(\lambda \mid \boldsymbol{x}_{n}\right)}
$$


Dado o modelo (2.3)-(2.4) e (B.1), podemos calcular $\mathbb{E}\left[L\left(\lambda, d_{n}^{*}\right) \mid \boldsymbol{x}_{n}\right]$.

\section{B.3 Algoritmos necessários para o cálculo do tamanho amostral}

\section{B.3.1 CCobM}

Um possível algoritmo para obtenção do tamanho amostral $n$ usando o CCobM é descrito como segue.

Passo 1. Fixe $n=1, \ell, \phi, \lambda_{0}, \theta_{0}, w$ e $\rho$;

Passo 2. Simule uma amostra de tamanho $M($ e.g., $M=1000)$ de $s_{n}$; para simular $s_{n}$, primeiramente simule uma amostra de tamanho $n$ de $\lambda$ da distribuição a priori $P V I\left(0, \phi / w, \theta_{0}, \theta_{0} / \lambda_{0}+\right.$ 1), e dados esses valores simule uma amostra de tamanho $n$ de $X_{i}$ da distribuição binomial negativa com média $w \lambda$ e parâmetro de dispersão $\phi$, de acordo com o modelo (2.3)-(2.4), em seguida some os $X_{i}$ 's simulados;

Passo 3. Calcule o limitante inferior a do intervalo HPD usando (B.2) e então a probabilidade a posteriori $\mathbb{P}\left[a \leq \lambda \leq a+\ell \mid s_{n}\right]$ usando (B.1) para cada $s_{n}$ simulado; para cada valor simulado no Passo 2, calcule $\theta_{0}+s_{n}$ e $\theta_{0} / \lambda_{0}+n \phi+1$ referentes à distribuição a posteriori Pearson Tipo VI de $\lambda$, e então calcule o limitante inferior $a$ do intervalo HPD usando (B.2). Em seguida, calcule as probabilidades a posteriori usando a distribuição a posteriori Pearson Tipo VI com parâmetros $\theta_{0}+s_{n}$ e $\theta_{0} / \lambda_{0}+n \phi+1($ A.1);

Passo 4. Calcule a média das $M$ probabilidades a posteriori;

Passo 5. Se essa média for $\geq 1-\rho$, pare. O valor $n$ fixado nesse passo é o valor desejado. Caso contrário, faça $n=n+1$ e volte ao Passo 2 .

\section{B.3.2 CCompM}

Um possível algoritmo para obtenção do tamanho amostral $n$ usando o CCompM é descrito como segue.

Passo 1. Fixe $n=1, \ell_{\max }, \phi, \lambda_{0}, \theta_{0}, w$ e $\rho$;

Passo 2. Simule uma amostra de tamanho $M($ e.g., $M=1000)$ de $s_{n}$; para simular $s_{n}$, primeiramente simule uma amostra de tamanho $n$ de $\lambda$ da distribuição a priori $P V I\left(0, \phi / w, \theta_{0}, \theta_{0} / \lambda_{0}+\right.$ 1), e dados esses valores simule, uma amostra de tamanho $n$ de $X_{i}$ da distribuição binomial 
negativa com média $w \lambda$ e parâmetro de dispersão $\phi$, de acordo com o modelo (2.3)-(2.4), em seguida some os $X_{i}$ 's simulados;

Passo 3. Calcule o comprimento do intervalo de credibilidade a posteriori $(1-\rho)$ para cada $s_{n}$ simulado; para cada valor simulado no Passo 2 , calcule $\theta_{0}+s_{n}$ e $\theta_{0} / \lambda_{0}+n \phi+1$ referentes à distribuição a posteriori Pearson Tipo VI de $\lambda$, e então obtenha os quantis de ordens $\rho / 2$ e $1-\rho / 2$. Em seguida, calcule a diferença entre os quantis para cada valor simulado a fim de obter o comprimento do intervalo de credibilidade;

Passo 4. Calcule a média dos $M$ comprimentos dos intervalos de credibilidade a posteriori;

Passo 5. Se essa média for $\leq \ell_{\max }$, pare. O valor $n$ fixado nesse passo é o valor desejado. Caso contrário, faça $n=n+1$ e volte ao Passo 2 .

\section{B.3.3 MCT}

Um possível algoritmo para obtenção do tamanho da amostra $n$ usando o MCT com a função perda 1 ou a 2 é descrito como segue.

Passo 1. Fixe $\lambda_{0}, \theta_{0}, \phi, w, c$, e $\rho$ (função perda 1 ) ou $\gamma$ (função perda 2), além de um conjunto na qual $n$ irá variar;

Passo 2. Para cada $n$, simule uma amostra de tamanho $M($ e.g., $M=1000)$ de $s_{n}\left(s_{n}\right.$ pode ser simulado da seguinte forma: simula-se uma amostra de tamanho $n$ de $\lambda$ da distribuição a priori $P V I\left(0, \phi / w, \theta_{0}, \theta_{0} / \lambda_{0}+1\right)$, com esses valores simula-se uma amostra de tamanho $n$ de $X_{i}, i=1, \ldots, n$ de uma distribuição binomial negativa com média $w \lambda$ e parâmetro $\phi$, e por último soma-se os valores $X_{i}$ ), calcule os respectivos limitantes $a$ e $b$ (no caso da função perda 1), em seguida os respectivos $\mathbb{E}\left[L\left(\lambda, d_{n}^{*}\right) \mid \boldsymbol{x}_{n}\right]$ usando (B.3) ou (B.4), e finalmente calcule a média desses $M$ valores esperados, guarde esse valor;

Passo 3. Para cada risco de Bayes a posteriori estimado, some o respectivo valor de custo cn, guarde esses valores;

Passo 4. Com os valores obtidos no Passo 3 e os respectivos valores de $n$, ajuste o modelo de regressão (3.5) e obtenha o valor mínimo de $n$ usando (3.6). 


\section{Apêndice $\mathrm{C}$}

\section{Modelo Poisson com distribuição a}

\section{priori não-paramétrica}

\section{C.1 Distribuição a posteriori e propriedades}

Em algumas situações sabe-se que a distribuição de probabilidade, $F$, dos dados em estudo pertence a um conjunto de distribuições, que pode ser tratado como um "espaço paramétrico". Uma maneira de restringir o conjunto de distribuições a priori nesse caso (e não considerar todas as possíveis distribuições sobre os reais) é considerar um processo Dirichlet (DP). O processo Dirichlet foi proposto por Freedman (1963) e suas propriedades foram desenvolvidas por Ferguson (1973). Em particular, Ferguson (1973) introduziu o processo Dirichlet como uma possível solução para o problema da especificação de distribuições a priori sob uma abordagem bayesiana não-paramétrica em que o espaço paramétrico é um conjunto de distribuições de probabilidade sobre um dado espaço amostral.

Primeiramente, definimos a distribuição Dirichlet de uma maneira mais geral do que a usual como fez Ferguson (1973). Seja $G(\kappa, \theta)$ a distribuição gama com parâmetro de forma $\kappa \geq 0$ e de escala $\theta>0$. Para $\kappa=0$, esta distribuição é degenerada no ponto zero; para $\kappa>0$, sua função densidade é

$$
f(z ; \kappa, \theta)=\frac{\theta^{\kappa}}{\Gamma(\kappa)} z^{\kappa-1} \exp (-\theta z), \quad z>0
$$

Sejam $Z_{1}, Z_{2}, \ldots, Z_{k}$ variáveis aleatórias independentes tais que $Z_{j} \sim G\left(\kappa_{j}, 1\right)$, em que $\kappa_{j} \geq 0$ com $\kappa_{j}>0$ para algum $j, j=1, \ldots, k$. A distribuição Dirichlet com parâmetro $\left(\kappa_{1}, \ldots, \kappa_{k}\right)$, 
denotada por $\operatorname{Dir}\left(\kappa_{1}, \ldots, \kappa_{k}\right)$, é a distribuição do vetor $\left(Y_{1}, \ldots, Y_{k}\right)$ em que

$$
Y_{j}=\frac{Z_{j}}{\sum_{i=1}^{k} Z_{i}}, \quad j=1, \ldots, k
$$

Nesta configuração, se algum $\kappa_{j}=0$, a correspondente variável $Y_{j}$ é degenerada no ponto zero. Por outro lado, se $\kappa_{j}>0 \forall j$, a função densidade do vetor $\left(Y_{1}, \ldots, Y_{k}\right)$ é

$$
f\left(y_{1}, \ldots, y_{k-1} ; \kappa_{1}, \ldots, \kappa_{k}\right)=\frac{\Gamma\left(\kappa_{1}+\cdots+\kappa_{k}\right)}{\Gamma\left(\kappa_{1}\right) \ldots \Gamma\left(\kappa_{k}\right)}\left(\prod_{j=1}^{k-1} y_{j}^{\kappa_{j}-1}\right)\left(1-\sum_{j=1}^{k-1} y_{j}\right)^{\kappa_{k}-1}
$$

em que $\left(y_{1}, \ldots, y_{k-1}\right) \in\left\{\left(y_{1}, \ldots, y_{k-1}\right): y_{j} \geq 0, \sum_{j=1}^{k-1} y_{j} \leq 1\right\}$. Isto implica que a distribuição marginal de cada $Y_{j}$ é $B\left(\kappa_{j}, \sum_{i=1}^{k} \kappa_{i}-\kappa_{j}\right)$ em que $B(a, b)$ denota a distribuição beta com parâmetros $a$ e $b$. Dado que $\left(Y_{1}, \ldots, Y_{k}\right) \sim \operatorname{Dir}\left(\kappa_{1}, \ldots \kappa_{k}\right)$, então

$$
\mathbb{E}\left[Y_{i}\right]=\frac{\kappa_{i}}{\kappa}, \quad \mathbb{E}\left[Y_{i}^{2}\right]=\frac{\kappa_{i}\left(\kappa_{i}+1\right)}{\kappa(\kappa+1)} \quad \text { e } \quad \mathbb{E}\left[Y_{i} Y_{j}\right]=\frac{\kappa_{i} \kappa_{j}}{\kappa(\kappa+1)},
$$

em que $\kappa=\sum_{i=1}^{k} \kappa_{i}$. Para mais detalhes sobre a distribuição Dirichlet, vide e.g. Ferguson (1973).

Definição C.1. (Ferguson, 1973) Seja $\Lambda$ um conjunto e $\mathcal{A}$ uma $\sigma$-álgebra de subconjuntos de $\Lambda$. Seja $\alpha(\cdot)$ uma medida finita não-nula (não-negativa e finitamente aditiva) em $(\Lambda, \mathcal{A})$. Dizemos que $F$ é um processo Dirichlet em $(\Lambda, \mathcal{A})$ com parâmetro $\alpha(\cdot)$ se $\forall k=1,2, \ldots$, e toda partição mensurável $\left(A_{1}, \ldots, A_{k}\right)$ de $\Lambda$, a distribuição de $\left[F\left(A_{1}\right), \ldots, F\left(A_{k}\right)\right]$ é Dirichlet com parâmetro $\left[\alpha\left(A_{1}\right), \ldots, \alpha\left(A_{k}\right)\right]$.

Proposição C.1. (Ferguson, 1973) Seja $F$ um processo Dirichlet em $(\Lambda, \mathcal{A})$ com parâmetro $\alpha(\cdot)$ $e A \in \mathcal{A}$. Então, $\mathbb{E}[F(A)]=\alpha(A) / \alpha(\Lambda) e \operatorname{Var}[F(A)]=\alpha(A)[\alpha(\Lambda)-\alpha(A)] / \alpha^{2}(\Lambda)[\alpha(\Lambda)+1]$.

Demonstração. Considere a partição $\left(A, A^{c}\right)$, e note que $F(A)$ segue uma distribuição $B\left(\alpha(A), \alpha\left(A^{c}\right)\right)$. Então,

$$
\mathbb{E}[F(A)]=\frac{\alpha(A)}{\alpha(A)+\alpha\left(A^{c}\right)}=\frac{\alpha(A)}{\alpha(\Lambda)}
$$

e

$$
\operatorname{Var}[F(A)]=\frac{\alpha(A) \alpha\left(A^{c}\right)}{\left[\alpha(A)+\alpha\left(A^{c}\right)\right]^{2}\left[\alpha(A)+\alpha\left(A^{c}\right)+1\right]}=\frac{\alpha(A)[\alpha(\Lambda)-\alpha(A)]}{\alpha^{2}(\Lambda)[\alpha(\Lambda)+1]} .
$$

Proposição C.2. (Ferguson, 1973) Seja F um processo Dirichlet em $(\Lambda, \mathcal{A})$ com parâmetro $\alpha(\cdot)$ e $\lambda$ uma amostra de tamanho 1 de $F$. Então, para $A \in \mathcal{A}, \mathbb{P}[\lambda \in A]=\alpha(A) / \alpha(\Lambda)$. 
Demonstração. Uma vez que $\mathbb{P}[\lambda \in A \mid F(A)]=F(A)$ quase certamente, então

$$
\mathbb{P}[\lambda \in A]=\mathbb{E}[\mathbb{P}[\lambda \in A \mid F(A)]]=\mathbb{E}[F(A)]=\frac{\alpha(A)}{\alpha(\Lambda)} .
$$

Definição C.2. Seja $\delta_{\lambda}(\cdot)$ uma medida sobre $(\Lambda, \mathcal{A}), \lambda \in \Lambda$, definida por $\delta_{\lambda}(A)=1$, se $\lambda \in A$, e $\delta_{\lambda}(A)=0$, em caso contrário.

Teorema C.1. (Ferguson, 1973) Seja $F$ um processo Dirichlet sobre $(\Lambda, \mathcal{A})$ com parâmetro $\alpha(\cdot)$ e seja $\lambda_{1}, \ldots, \lambda_{n}$ uma amostra de tamanho $n$ de $F$. Então, a distribuição condicional de $F$ dados $\lambda_{1}, \ldots, \lambda_{n}$ é um processo Dirichlet com parâmetro $\alpha(\cdot)+\sum_{i=1}^{n} \delta_{\lambda_{i}}(\cdot)$.

Demonstração. Vide Ferguson (1973, pp. 217-218).

Blackwell \& MacQueen (1973) apresentam uma interessante conexão entre o processo Dirichlet e o modelo de urna de Pólya. Sejam $\alpha^{*}>0$ e $F_{0}$ uma medida de probabilidade sobre $\Lambda$. Uma sequência de variáveis aleatórias $\lambda_{1}, \lambda_{2}, \ldots$ é proveniente de um modelo de urna de Pólya com parâmetro $\alpha^{*} F_{0}(\cdot)$ se $\forall A \in \mathcal{A}$,

$$
\mathbb{P}\left[\lambda_{1} \in A\right]=F_{0}(A) \quad \text { e } \quad \mathbb{P}\left[\lambda_{n} \in A \mid \lambda_{1}, \ldots, \lambda_{n-1}\right]=\frac{\alpha^{*} F_{0}(A)+\sum_{i=1}^{n-1} \delta_{\lambda_{i}}(A)}{\alpha^{*}+n-1}
$$

para $n=2,3, \ldots$. Blackwell \& MacQueen (1973) mostram que $\left[\alpha^{*} F_{0}+\sum_{i=1}^{n-1} \delta_{\lambda_{i}}\right] /\left(\alpha^{*}+n-1\right)$ converge quase certamente para um processo Dirichlet com parâmetro $\alpha^{*} F_{0}(\cdot)$ à medida que $n \rightarrow \infty$.

Note que nesta construção, o processo Dirichlet depende de dois parâmetros $\left(\alpha^{*}\right.$ e $\left.F_{0}\right)$, diferentemente da construção de Ferguson (1973) em que depende somente de um parâmetro, a medida $\alpha(\cdot)$. Os três parâmetros se relacionam por meio de $\alpha\left(\cdot ; \alpha^{*}, F_{0}\right)=\alpha^{*} F_{0}(\cdot)$. A função distribuição $F_{0}$ é usualmente chamada de distribuição base e note que $\alpha\left(\Lambda ; \alpha^{*}, F_{0}\right)=\alpha^{*} F_{0}(\Lambda)=\alpha^{*}$. Usando a Proposição C.1, concluímos que $\mathbb{E}[F(A)]=F_{0}(A)$ e $\operatorname{Var}[F(A)]=F_{0}(A)\left[1-F_{0}(A)\right] /\left(\alpha^{*}+1\right)$, em que $A \in \mathcal{A}$. À medida que $\alpha^{*}$ cresce, há uma maior concentração de $F(A)$ em torno de $F_{0}(A)$. Nesse sentido, pode haver uma confusão em dizer que à medida que $\alpha^{*} \rightarrow \infty(0)$ temos cada vez mais (menos) informação a respeito da função distribuição em estudo, o que não é totalmente verdade. O que acontece é que enquanto a probabilidade $F(A)$ aumenta (diminui), a probabilidade $F\left(A^{c}\right)$ diminui (aumenta), pois a covariância entre esses dois é

$$
\operatorname{Cov}\left(F(A), F\left(A^{c}\right)\right)=-\frac{F_{0}(A) F_{0}\left(A^{c}\right)}{\alpha^{*}+1}
$$


Assim, o que poderíamos dizer é que à medida que $\alpha^{*} \rightarrow \infty$, temos cada vez mais informação em $A$ e menos em $A^{c}$, i.e., uma espécie de informação "local", e não informação "global" sobre todo o espaço paramétrico. Sethuraman \& Tiwari (1981) mostraram que $D P\left[\alpha^{*} F_{0}(\cdot)\right] \rightarrow \delta_{\lambda}\left(\lambda_{0}\right)$ em distribuição quando $\alpha^{*} \rightarrow 0$, em que $\lambda_{0} \sim F_{0}$. Assim, quanto mais próximo de zero $\alpha^{*}$ estiver, mais informação "global" sobre a função distribuição em estudo e sua respectiva variável teremos.

Considere o seguinte modelo:

$$
\begin{aligned}
& X_{i} \mid \lambda_{i} \stackrel{\mathrm{ind}}{\sim} g\left(x_{i} \mid \lambda_{i}\right), \quad i=1,2, \ldots, n ; \\
& \lambda_{i} \mid F \stackrel{\mathrm{iid}}{\sim} F, \quad i=1,2, \ldots, n ; \\
& F \sim D P\left[\alpha\left(\cdot ; \alpha^{*}, F_{0}\right)\right],
\end{aligned}
$$

em que $D P\left[\alpha\left(\cdot ; \alpha^{*}, F_{0}\right)\right]$ é um processo Dirichlet com parâmetro $\alpha\left(\cdot ; \alpha^{*}, F_{0}\right)=\alpha^{*} F_{0}(\cdot)$ e $g(x \mid \lambda)$ é a função densidade (ou de probabilidade) condicional de $X$ dado $\lambda$. O modelo (C.6)-(C.8) é conhecido como mistura por processo Dirichlet. Considerando $n=1$, a função densidade (ou de probabilidade) marginal de $X$ é dada por

$$
g(x)=\mathbb{E}\left[\int_{\Lambda} g(x \mid \lambda) F(d \lambda)\right]
$$

em que o valor esperado é calculado sobre o processo Dirichlet. Pela Proposição C.1 (Hjort et al., 2010, p. 39), temos

$$
g(x)=\int_{\Lambda} g(x \mid \lambda) \mathbb{E}[F(d \lambda)]=\int_{\Lambda} g(x \mid \lambda) F_{0}(d \lambda),
$$

que também é o estimador de Bayes da função densidade (ou de probabilidade) marginal de $X$ com respeito à perda quadrática para o problema "sem amostra" na estimação de densidades sob o modelo (C.6)-(C.8) (Lo, 1984). Este resultado é útil para simular amostras da distribuição marginal de $X_{i}$, uma vez que estamos assumindo que as contagens observadas são independentes, podemos simular uma amostra de tamanho $n$ simulando cada observação através de $g(x)$. Para o modelo (2.5)-(2.7) com $F_{0}$ sendo uma distribuição gama fica mais fácil a simulação de amostras, pois a distribuição marginal de $X_{i}$ será binomial negativa com média $w \lambda_{0}$ e parâmetro de dispersão $\theta_{0}$.

A distribuição a posteriori de $F$ sob o modelo (C.6)-(C.8) é dada por (Antoniak, 1974; Ishwaran \& Zarepour, 2002)

$$
F\left(A \mid \boldsymbol{x}_{n}\right)=\int_{\Lambda^{n}} F\left(A \mid \boldsymbol{\lambda}_{n}\right) \nu\left(d \boldsymbol{\lambda}_{n} \mid \boldsymbol{x}_{n}\right)
$$


em que $A \in \mathcal{A}, \boldsymbol{\lambda}_{n}=\left(\lambda_{1}, \ldots, \lambda_{n}\right), F\left(\cdot \mid \boldsymbol{\lambda}_{n}\right)$ é um processo Dirichlet com parâmetro $\alpha\left(\cdot ; \alpha^{*}, F_{0}, \boldsymbol{\lambda}_{n}\right)=$ $\alpha^{*} F_{0}(\cdot)+\sum_{i=1}^{n} \delta_{\lambda_{i}}(\cdot), \boldsymbol{x}_{n}=\left(x_{1}, \ldots, x_{n}\right) \mathrm{e}$

$$
\nu\left(d \boldsymbol{\lambda}_{n} \mid \boldsymbol{x}_{n}\right) \propto \prod_{i=1}^{n} g\left(x_{i} \mid \lambda_{i}\right)\left[\alpha^{*} F_{0}\left(d \lambda_{i}\right)+\sum_{j=1}^{i-1} \delta_{\lambda_{i}}\left(d \lambda_{j}\right)\right] .
$$

Esta expressão de $\nu\left(d \boldsymbol{\lambda}_{n} \mid \boldsymbol{x}_{n}\right)$ resulta da construção por meio do modelo de urna de Pólya (C.5) que leva a um processo Dirichlet. Para mais detalhes, vide e.g. Antoniak (1974). Para simular uma amostra de $\nu\left(d \boldsymbol{\lambda}_{n} \mid \boldsymbol{x}_{n}\right)$, considere a seguinte distribuição condicional completa (Escobar \& West, 1998; MacEachern \& Müller, 1998):

$$
\nu\left(d \lambda_{i} \mid \boldsymbol{\lambda}_{(-i)}, \boldsymbol{x}_{n}\right) \propto q_{0} g\left(x_{i} \mid \lambda_{i}\right) F_{0}\left(d \lambda_{i}\right)+\sum_{j \neq i} q_{j} \delta_{\lambda_{j}}\left(d \lambda_{i}\right),
$$

em que $\boldsymbol{\lambda}_{(-i)}=\left\{\lambda_{j} \mid j \neq i, j=1, \ldots, n\right\} \mathrm{e}$

$$
q_{0} \propto \alpha^{*} \int_{\Lambda} g\left(x_{i} \mid \lambda_{i}\right) F_{0}\left(d \lambda_{i}\right) \quad \text { e } \quad q_{j} \propto g\left(x_{i} \mid \lambda_{j}\right)
$$

Os termos $q_{j}, j=0,1, \ldots, n, j \neq i$ são padronizadas de maneira que $q_{0}+\sum_{j \neq i} q_{j}=1$. Esse resultado nos permite estruturar um algoritmo de Gibbs para simular uma amostra de $\boldsymbol{\lambda}_{n}$ dado $\boldsymbol{x}_{n}$. Para o modelo (2.5)-(2.7), o termo $q_{0}$ pode ser facilmente calculado, pois a respectiva integral resultará na função de probabilidade de uma variável aleatória binomial negativa. Um algoritmo está apresentado em C.3.1. Um critério simples para monitorar o desempenho do algoritmo é a estabilização do número de valores diferentes para $\lambda$ ("clusters") (Kalli et al., 2011; Papaspiliopoulos \& Roberts, 2008). Esse resultado também é útil para estimar integrais calculadas com respeito à (C.11).

Como $F\left(A \mid \boldsymbol{\lambda}_{n}\right)$ é um processo Dirichlet, pela Proposição (C.1) temos que $\mathbb{E}\left[F\left(A \mid \boldsymbol{\lambda}_{n}\right)\right]=$ $\left[\alpha^{*} F_{0}(A)+n F_{n}(A)\right] /\left(\alpha^{*}+n\right)$, em que $n F_{n}(A)=\sum_{i=1}^{n} \delta_{\lambda_{i}}(A)$. Logo, de (C.10) obtemos

$$
\mathbb{E}\left[F\left(A \mid \boldsymbol{x}_{n}\right)\right]=\int_{\Lambda^{n}} \mathbb{E}\left[F\left(A \mid \boldsymbol{\lambda}_{n}\right)\right] \nu\left(d \boldsymbol{\lambda}_{n} \mid \boldsymbol{x}_{n}\right)=\frac{\alpha^{*}}{\alpha^{*}+n} F_{0}(A)+\frac{n}{\alpha^{*}+n} \int_{\Lambda^{n}} F_{n}(A) \nu\left(d \boldsymbol{\lambda}_{n} \mid \boldsymbol{x}_{n}\right) .
$$

Uma maneira prática para se obter uma representação mais interpretável para (C.13) é considerar um reticulado $t_{1}<t_{2}<\cdots<t_{K}$ de valores sobre o conjunto dos reais e calcular a probabilidade acumulada até cada ponto (inclusive), i.e., considerar intervalos semi-abertos e exaustivos em vez de um conjunto $A$ genérico (Ishwaran \& James, 2002). Para calcular (C.13) para um dado $A$, necessitamos calcular a integral da expressão, nesse sentido se faz necessário utilizar métodos de Monte Carlo para obter uma estimativa dessa integral basicamente como segue: simule $\boldsymbol{\lambda}_{n}$ de 
$\nu\left(\cdot \mid \boldsymbol{x}_{n}\right)$ e em seguida calcule a proporção de $\lambda_{i}$ 's de $\boldsymbol{\lambda}_{n}$ que pertencem a $A$, repita o procedimento anterior um número determinado de vezes (e.g., 1000), em seguida calcule a média dos valores obtidos anteriormente e essa média será a estimativa da integral. Note que a mesma ideia pode ser usada para calcular integrais de funções de $F_{n}(A)$ em relação à $\nu\left(\cdot \mid \boldsymbol{x}_{n}\right)$.

\section{C.2 Obtenção da decisão de Bayes usando MCT}

\section{C.2.1 Função perda 3}

Obtemos a decisão de Bayes considerando a função perda 3 da seguinte forma. Calculamos a decisão de Bayes para o problema "sem amostra" e em seguida estendemos para o problema com uma amostra de tamanho n. O risco de Bayes para o problema "sem amostra" é calculado tomando-se o valor esperado da função perda em relação ao processo Dirichlet fixado como distribuição a priori, i.e.,

$$
\mathbb{E}\left[L\left(F, D_{0}\right)\right]=\int_{-\infty}^{\infty} \mathbb{E}\left[\left(F\left(A_{t}\right)-D_{0}\left(A_{t}\right)\right)^{2}\right] W(d t)
$$

Logo, a decisão de Bayes neste caso será $D_{0}^{*}\left(A_{t}\right)=\mathbb{E}\left[F\left(A_{t}\right)\right]=F_{0}\left(A_{t}\right)$. Dadas observações resultantes de uma mistura por processo Dirichlet, então a decisão de Bayes para o problema com uma amostra de tamanho $n$ é

$$
D_{n}^{*}\left(A_{t}\right)=\mathbb{E}\left[F\left(A_{t} \mid \boldsymbol{x}_{n}\right)\right]=\frac{\alpha^{*}}{\alpha^{*}+n} F_{0}\left(A_{t}\right)+\frac{n}{\alpha^{*}+n} \int_{\Lambda^{n}} F_{n}\left(A_{t}\right) \nu\left(d \boldsymbol{\lambda}_{n} \mid \boldsymbol{x}_{n}\right)
$$

pois $F\left(A_{t} \mid \boldsymbol{\lambda}_{n}\right)$ é um processo Dirichlet e $F\left(A_{t} \mid \boldsymbol{x}_{n}\right)$ é uma mistura de processos Dirichlet. Logo,

$$
\mathbb{E}\left[L\left(F, D_{n}^{*}\right) \mid \boldsymbol{x}_{n}\right]=\int_{-\infty}^{\infty} \mathbb{E}\left[\left(F\left(A_{t} \mid \boldsymbol{x}_{n}\right)-D_{n}^{*}\left(A_{t}\right)\right)^{2}\right] W(d t)=\int_{-\infty}^{\infty} \operatorname{Var}\left[F\left(A_{t} \mid \boldsymbol{x}_{n}\right)\right] W(d t) .
$$

Precisamos agora obter uma expressão para $\operatorname{Var}\left[F\left(A_{t} \mid \boldsymbol{x}_{n}\right)\right]$. Primeiramente, note que dado que $F\left(A_{t} \mid \boldsymbol{\lambda}_{n}\right)$ é um processo Dirichlet com parâmetro $\alpha\left(A_{t} ; \alpha^{*}, F_{0}, \boldsymbol{\lambda}_{n}\right)=\alpha^{*} F_{0}\left(A_{t}\right)+\sum_{i=1}^{n} \delta_{\lambda_{i}}\left(A_{t}\right)$, então, pela Proposição (C.1) temos que

$$
\begin{aligned}
\operatorname{Var}\left[F\left(A_{t} \mid \boldsymbol{\lambda}_{n}\right)\right] & =\frac{1}{\alpha^{*}+n+1}\left\{\frac{\alpha^{*} F_{0}\left(A_{t}\right)+n F_{n}(A)}{\alpha^{*}+n}\left[1-\frac{\alpha^{*} F_{0}\left(A_{t}\right)+n F_{n}\left(A_{t}\right)}{\alpha^{*}+n}\right]\right\} \\
& =\frac{1}{\left(\alpha^{*}+n+1\right)\left(\alpha^{*}+n\right)^{2}}\left\{\left(\alpha^{*}\right)^{2} F_{0}\left(A_{t}\right)\left[1-F_{0}\left(A_{t}\right)\right]+\alpha^{*} n F_{0}\left(A_{t}\right)\right. \\
& \left.+n^{2} F_{n}\left(A_{t}\right)\left[1-F_{n}\left(A_{t}\right)\right]+\alpha^{*} n F_{n}\left(A_{t}\right)\left[1-2 F_{0}\left(A_{t}\right)\right]\right\} .
\end{aligned}
$$


Logo, de (C.10) obtemos

$$
\begin{aligned}
\operatorname{Var}\left[F\left(A_{t} \mid \boldsymbol{x}_{n}\right)\right] & =\frac{1}{\left(\alpha^{*}+n+1\right)\left(\alpha^{*}+n\right)^{2}}\left\{\left(\alpha^{*}\right)^{2} F_{0}\left(A_{t}\right)\left[1-F_{0}\left(A_{t}\right)\right]+\alpha^{*} n F_{0}\left(A_{t}\right)\right. \\
& \left.+n^{2} \int_{\Lambda^{n}} F_{n}\left(A_{t}\right)\left[1-F_{n}\left(A_{t}\right)\right] \nu\left(d \boldsymbol{\lambda}_{n} \mid \boldsymbol{x}_{n}\right)+\alpha^{*} n\left[1-2 F_{0}\left(A_{t}\right)\right] \int_{\Lambda^{n}} F_{n}\left(A_{t}\right) \nu\left(d \boldsymbol{\lambda}_{n} \mid \boldsymbol{x}_{n}\right)\right\} .
\end{aligned}
$$

Note que a primeira integral de $\operatorname{Var}\left[F\left(A_{t} \mid \boldsymbol{x}_{n}\right)\right]$ pode ser estimada através do mesmo algoritmo usado para estimar a segunda integral (comentado no final da Seção C.1), a diferença aqui será que ao invés de calcular somente a proporção de $\lambda_{i}$ 's de $\boldsymbol{\lambda}_{n}$ (simulado de $\nu\left(\cdot \mid \boldsymbol{x}_{n}\right)$ ) que pertencem a $A_{t}$, calculamos também proporção $\times(1$ - proporção $)$. O restante da expressão pode ser facilmente calculado com o conhecimento de $F_{0}$. $\mathrm{O}$ algoritmo para obter uma estimativa dessa variância é similar ao C.3.2 com a diferença que neste caso temos que estimar duas integrais em relação a $\nu\left(\cdot \mid \boldsymbol{x}_{\boldsymbol{n}}\right)$.

\section{C.3 Algoritmos necessários para o cálculo do tamanho amostral}

\section{C.3.1 Distribuição $\nu\left(\cdot \mid \boldsymbol{x}_{n}\right)$}

Um possível algoritmo para simular uma amostra da distribuição $\nu\left(\cdot \mid \boldsymbol{x}_{n}\right)$ e que também pode ajudar na estimação de integrais calculadas em relação a esta distribuição é descrito como segue.

Passo 1. Simule $\lambda_{i}$ de $F_{0}, i=1, \ldots, n$;

Passo 2. Para $i=1, \ldots, n$ :

1. Calcule $q_{0}$ e $q_{j}, j=1, \ldots, n$ conforme (C.12) e padronize-os;

2. Com probabilidade $q_{0}$ simule $\lambda_{i}$ de $g\left(x_{i} \mid \lambda_{i}\right) F_{0}\left(d \lambda_{i}\right)$ ou de $\delta_{\lambda_{j}}\left(d \lambda_{i}\right)$ com probabilidade $q_{j}$, $j \neq i$.

Passo 3. Repita o passo anterior até atingir um critério de parada, e.g., a estabilização do número de valores diferentes para $\lambda_{i}$.

\section{C.3.2 Estimação de (C.13)}

Dado $\boldsymbol{x}_{n}$ e um conjunto $A$, um possível algoritmo para calcular uma estimativa de (C.13) é descrito como segue.

Passo 1. Simule $M$ amostras (e.g., $M=100$ ) de $\boldsymbol{\lambda}_{n}$ usando o algoritmo da Seção C.3.1; 
Passo 2. Calcule uma estimativa da integral em (C.13) da seguinte forma: Para cada amostra $\boldsymbol{\lambda}_{n}$ calcule a proporção de $\lambda_{i}$ 's que pertence ao conjunto $A$. Em seguida calcule a média das $M$ proporções, essa será uma estimativa da integral;

Passo 3. Multiplique a estimativa da integral obtida no Passo 2 por $n /\left(\alpha^{*}+n\right)$, calcule $F_{0}(A)$ e em seguida multiplique-o por $\alpha^{*} /\left(\alpha^{*}+n\right)$, finalmente some os dois resultados obtidos.

\section{C.3.3 Obtenção da região HPD de probabilidade $1-\rho$}

Dado $\boldsymbol{x}_{n}$ um possível algoritmo para obter a região HPD de probabilidade $1-\rho$ usando a distribuição (C.13) é descrito como segue.

Passo 1. Considere um reticulado $t_{1}<t_{2}<\cdots<t_{K}$ do espaço paramétrico, com $K$ um número arbitrário fixado e considere $A_{t_{i}}=\left(-\infty, t_{i}\right], i=1,2, \ldots, K$;

Passo 2. Estime as probabilidades $\mathbb{E}\left[F\left(A_{t_{i}} \mid \boldsymbol{x}_{n}\right)\right], i=1,2, \ldots, K$ usando o algoritmo da Seção C.3.2, em seguida calcule a probabilidade $\mathbb{E}\left[F\left(A_{t_{i}} \mid \boldsymbol{x}_{n}\right)\right]-\mathbb{E}\left[F\left(A_{t_{i-1}} \mid \boldsymbol{x}_{n}\right)\right]$, referente ao intervalo $\left(t_{i-1}, t_{i}\right], i=1,2, \ldots, K$

Passo 3. Obtenha o intervalo $\left(t_{i-1}, t_{i}\right]$ com maior probabilidade dentre as probabilidades calculadas no Passo 2;

Passo 4. Guarde o intervalo obtido no Passo 3 e sua respectiva probabilidade;

Passo 5. Se a soma das probabilidades guardadas no Passo 4 for $\geq 1-\rho$, pare. A união dos intervalos guardados é a região HPD desejada e seu tamanho a soma dos tamanhos dos respectivos intervalos guardados no Passo 4. Caso contrário, volte ao Passo 3 e desconsidere o último intervalo obtido quando for obter o próximo intervalo com maior probabilidade.

\section{C.3.4 Obtenção da região HPD de tamanho $\ell$}

Dado $\boldsymbol{x}_{n}$, um possível algoritmo para obter a região HPD de tamanho $\ell$ usando a distribuição (C.13) é descrito como segue.

Passo 1. Considere um reticulado $t_{1}<t_{2}<\cdots<t_{K}$ do espaço paramétrico, com $K$ um número arbitrário fixado e considere $A_{t_{i}}=\left(-\infty, t_{i}\right], i=1,2, \ldots, K$;

Passo 2. Estime as probabilidades $\mathbb{E}\left[F\left(A_{t_{i}} \mid \boldsymbol{x}_{n}\right)\right], i=1,2, \ldots, K$ usando o algoritmo da Seção C.3.2, em seguida calcule a probabilidade $\mathbb{E}\left[F\left(A_{t_{i}} \mid \boldsymbol{x}_{n}\right)\right]-\mathbb{E}\left[F\left(A_{t_{i-1}} \mid \boldsymbol{x}_{n}\right)\right]$, referente ao intervalo $\left(t_{i-1}, t_{i}\right], i=1,2, \ldots, K$ 
Passo 3. Obtenha o intervalo $\left(t_{i-1}, t_{i}\right]$ com maior probabilidade dentre as probabilidades calculadas no Passo 2;

Passo 4. Guarde o intervalo obtido no Passo 3 e sua respectiva probabilidade;

Passo 5. Se a soma dos tamanhos dos intervalos guardados no Passo 4 for $\geq \ell$, pare. A união dos intervalos guardados é a região HPD desejada e sua probabilidade será a soma das respectivas probabilidades guardadas no Passo 4. Caso contrário, volte ao Passo 3 e desconsidere o último intervalo obtido quando for obter o próximo intervalo com maior probabilidade.

\section{C.3.5 CCobM}

Um algoritmo para obtenção do tamanho amostral $n$ usando o CCobM é descrito como segue.

Passo 1. Fixe $n=1, \ell, \lambda_{0}, \theta_{0}, w$ e $\rho$;

Passo 2. Simule $M$ amostras (e.g., $M=100)$ de $\boldsymbol{x}_{n}$; cada $x_{i}$ de $\boldsymbol{x}_{n}$ pode ser simulado de uma distribuição binomial negativa com média $w \lambda_{0}$ e parâmetro de dispersão $\theta_{0}$ pelo fato apresentado em (C.9) e de as variáveis $X_{i}$ serem consideradas independentes;

Passo 3. Para cada $\boldsymbol{x}_{n}$ simulado, obtenha a probabilidade da região HPD de tamanho $\ell$ usando o algoritmo da Seção C.3.4. Em seguida, calcule a média dessas $M$ probabilidades;

Passo 4. Se essa média for $\geq 1-\rho$, pare. O valor $n$ fixado nesse passo é o valor desejado. Caso contrário, faça $n=n+1$ e volte ao Passo 2 .

\section{C.3.6 CCompM}

Um algoritmo para obtenção do tamanho amostral $n$ usando o CCompM é descrito como segue.

Passo 1. Fixe $n=1, \ell_{\max }, \lambda_{0}, \theta_{0}, w$ e $\rho$;

Passo 2. Simule $M$ amostras (e.g., $M=100)$ de $\boldsymbol{x}_{n}$; cada $x_{i}$ de $\boldsymbol{x}_{n}$ pode ser simulado de uma distribuição binomial negativa com média $w \lambda_{0}$ e parâmetro de dispersão $\theta_{0}$ pelo fato apresentado em (C.9) e de as variáveis $X_{i}$ serem consideradas independentes;

Passo 3. Para cada $\boldsymbol{x}_{n}$ simulado obtenha o tamanho da região HPD de probabilidade $1-\rho$ usando o algoritmo da Seção C.3.3. Em seguida, calcule a média desses $M$ tamanhos;

Passo 4. Se essa média for $\leq \ell_{\max }$, pare. O valor $n$ fixado nesse passo é o valor desejado. Caso contrário, faça $n=n+1$ e volte ao Passo 2 . 


\section{C.3.7 MCT}

Um possível algoritmo para obtenção do tamanho da amostra $n$ usando o MCT com a função perda 3 é descrito como segue.

Passo 1. Fixe $\lambda_{0}, \theta_{0}, \alpha^{*}, w, c$, e a função de ponderação $W(t)$, além de um conjunto na qual $n$ irá variar;

Passo 2. Para cada $n$, simule uma amostra de tamanho $M($ e.g., $M=100)$ de $\boldsymbol{x}_{n}$, em seguida para cada $\boldsymbol{x}_{n}$ simulado calcule uma estimativa de $\mathbb{E}\left[L\left(F, D_{n}^{*}\right) \mid \boldsymbol{x}_{n}\right]$ da seguinte forma: considere um reticulado $t_{1}<t_{2}<\cdots<t_{K}$ do espaço paramétrico, com $K$ um número arbitrário fixado e considere $A_{t_{i}}=\left(t_{i}, t_{i-1}\right], i=1,2, \ldots, K$, obtenha uma estimativa de $\operatorname{Var}\left[F\left(A_{t_{i}} \mid \boldsymbol{x}_{n}\right)\right]$ e calcule $W\left(t_{i}\right)$, em seguida multiplique esses dois valores, para $i=1,2, \ldots, K$, em seguida calcule a soma desses produtos. Essa soma será a estimativa do valor esperado a posteriori da perda. A estimativa do risco de Bayes a posteriori minimizado será a média das $M$ estimativas dos valores esperados a posteriori da perda;

Passo 3. Para cada risco de Bayes a posteriori estimado, some o respectivo valor de custo cn, guarde esses valores;

Passo 4. Com os valores obtidos no Passo 3 e os respectivos valores de $n$, ajuste o modelo de regressão (3.5) e obtenha o valor mínimo de $n$ usando (3.6). 


\section{Referências}

AdCock, C. J. (1987). A Bayesian approach to calculating sample sizes for multinomial sampling. Journal of the Royal Statistical Society: Series D (The Statistician) 36, 155-159. 15

AdCOCK, C. J. (1988). A Bayesian approach to calculating sample sizes. Journal of the Royal Statistical Society: Series D (The Statistician) 37, 433-439. 15

Adcock, C. J. (1997). Sample size determination: a review. Journal of the Royal Statistical Society: Series D (The Statistician) 46, 261-283. 16

Aguirre-Macedo, M. L., Vidal-Martinez, V. M., Herrera-Silveira, J. A., ValdésLozano, D. S., Herrera-Rodríguez, M. \& Olvera-Novoa, M. A. (2008). Ballast water as a vector of coral pathogens in the Gulf of Mexico: The case of the cayo arcas coral reef. Marine Pollution Bulletin 56, 1570-1577. 34

Antoniak, C. E. (1974). Mixtures of Dirichlet processes with applications to Bayesian nonparametric problems. The Annals of Statistics 2, 1152-1174. 13, 14, 23, 24, 54, 55

Balaji, R., YaAkob, O. \& KoH, K. K. (2014). A review of developments in ballast water management. Environmental Reviews 22, 298-310. 35

Basurko, O. C. \& Mesbahi, E. (2011). Statistical representativeness of ballast water sampling. Em Proceedings of the Institution of Mechanical Engineer, Part M. Journal of Engineering for the Maritime Environment, 183-190. 1

Bernardo, J. M. (1997). Statistical inference as a decision problem: the choice of sample size. Journal of the Royal Statistical Society. Series D (The Statistician) 46, 151-153. 16

Bierman, S. M., VRies, P. \& KaAg, N. H. B. M. (2012). The development of a full standard methodology for testing ballast water discharges for gross non-compliance of the IMO's Ballast Water Management Convention (EMSA/NEG/12/2012). Relatório técnico C124/12, IMARES Wageningen UR. 1

Billingsley, P. (1995). Probability and Measure. New York: John Wiley \& Sons. 40

Blackwell, D. \& MacQueen, J. B. (1973). Ferguson distributions via Pólya-urn schemes. The Annals of Statistics 1, 353-355. 53

Brutti, P., De Santis, F. \& Gubbiotti, S. (2008). Robust Bayesian sample size determination in clinical trials. Statistics in Medicine 27, 2290-2306. 16

Brutti, P., De Santis, F. \& Gubbiotti, S. (2009). Mixtures of prior distributions for predictive Bayesian sample size calculations in clinical trials. Statistics in Medicine 28, 2185-2201. 16

Brutti, P., De Santis, F. \& Gubbiotti, S. (2014). Predictive measures of the conflict between frequentist and Bayesian estimators. Journal of Statistical Planning and Inference 148, 111-122. 16 
Bush, C. A. \& MacEachern, S. N. (1996). A semiparametric Bayesian model for randomised block designs. Biometrika 83, 275-285. 13

Carney, K. J., Basurko, O. C., Pazouki, K., Marsham, S., Delany, J. E., Desai, D. V., Anil, A. C. \& Mesbahi, E. (2013). Difficulties in obtaining representative samples for compliance with the Ballast Water Management Convention. Marine Pollution Bulletin 68, 99-105. 1

Chen, X. (2007). Exact computation of minimum sample size for estimation of Poisson parameters. http://arxiv.org/abs/0707.2116. Accessed March 20, 2017. 5

Chen, X. (2008). A simple sample size formula for estimating means of Poisson random variables. http://arxiv.org/abs/0804.3033. Accessed March 20, 2017. 5, 6

Chen, X. (2011). Exact computation of minimum sample size for estimation of binomial parameters. Journal of Statistical Planning and Inference 141, 2622-2632. 5

Chen, Z. \& Chen, X. (2016). Exact calculation of minimum sample size for estimating a Poisson parameter. Communications in Statistics-Theory and Methods 45, 4692-4715. 5

Chu, K. H., Tam, P. F., Fung, C. H. \& Chen, Q. C. (1997). A biological survey of ballast water in container ships entering Hong Kong. Hydrobiologia 352, 201-206. 35

Costa, E. G. (2013). Aspectos estatísticos da amostragem de água de lastro. Dissertação de mestrado. Departamento de Estatística, Universidade de São Paulo, São Paulo. 1

Costa, E. G., Lopes, R. M. \& Singer, J. M. (2015). Implications of heterogeneous distributions of organisms on ballast water sampling. Marine Pollution Bulletin 91, 280-287. 2, 3, 4, 15

Costa, E. G., Lopes, R. M. \& Singer, J. M. (2016). Sample size for estimating the mean concentration of organisms in ballast water. Journal of Environmental Management 180, 433-438. $2,4,5,6,15,25,30$

David, M., Gollasch, S., Cabrini, M., Perkovič, M., Bosnjak, D. \& Virgilio, D. (2007). Results from the first ballast water sampling study in the Mediterranean Sea, the Port of Koper study. Marine Pollution Bulletin 54, 53-65. 1

De Santis, F. (2007). Using historical data for Bayesian sample size determination. Journal of the Royal Statistical Society. Series A (Statistics in Society) 170, 95-113. 16

De Santis, F. \& Gubbiotti, S. (2016). A decision-theoretic approach to sample size determination under several priors. Applied Stochastic Models in Business and Industry, doi: 10.1002/asmb.2211. 16

Drake, D. A. R. \& Mandrak, N. E. (2010). Least-cost transportation networks predict spatial interaction of invasion vectors. Ecological Applications 20, 2286-2299. 35

Drake, D. A. R. \& Mandrak, N. E. (2014). Ecological risk of live bait fisheries: a new angle on selective fishing. Fisheries 39, 201-211. 35

Escobar, M. D. (1994). Estimating normal means with a Dirichlet process prior. Journal of The American Statistical Association 89, 268-277. 13

Escobar, M. D. \& West, M. (1995). Bayesian density estimation and inference using mixtures. Journal of The American Statistical Association 90, 577-588. 13

Escobar, M. D. \& West, M. (1998). Computing nonparametric hierarchical models. Em Practical nonparametric and semiparametric Bayesian Statistics. Springer, 1-22. 55 
Etzioni, R. \& Kadane, J. B. (1993). Optimal experimental design for another's analysis. Journal of the American Statistical Association 88, 1404-1411. 16

Faulkner, K. T., Robertson, M. P., Rouget, M. \& Wilson, J. R. (2014). A simple, rapid methodology for developing invasive species watch lists. Biological Conservation 179, 25-32. 35

Ferguson, T. S. (1973). A Bayesian analysis of some nonparametric problems. The Annals of Statistics 1, 209-230. 7, 13, 51, 52, 53

Freedman, D. A. (1963). On the asymptotic behavior of Bayes' estimates in the discrete case. The Annals of Mathematical Statistics 34, 1386-1403. 51

Gollasch, S. \& Leppäkoski, E. (2007). Risk assessment and management scenarios for ballast water mediated species introductions into the Baltic Sea. Aquatic Invasions 2, 313-340. 35

Hjort, N. L., Holmes, C., Müller, P. \& Walker, S. G. (2010). Bayesian Nonparametrics, $1^{\text {a }}$ ed. Cambridge: Cambridge University Press. 54

Ishwaran, H. \& James, L. F. (2002). Approximate Dirichlet process computing finite normal mixtures: smoothing and prior information. Journal of Computational and Graphical Statistics 11, 508-532. 55

IshWARAN, H. \& Zarepour, M. (2002). Exact and aproximate sum representations for the Dirichlet process. The Canadian Journal of Statistics 30, 269-283. 54

Islam, A. F. M. (2011). Loss functions, utility functions and Bayesian sample size determination. Tese de doutorado. Queen Mary, University of London. 16

Islam, A. F. M. S. \& Pettit, L. I. (2012). Bayesian sample size determination using linex loss and linear cost. Communications in Statistics-Theory and Methods 41, 223-240. 16

Islam, A. F. M. S. \& Pettit, L. I. (2014). Bayesian sample size determination for the bounded linex loss function. Journal of Statistical Computation and Simulation 84, 1644-1653. 16, 19

Johnson, N. L., Kotz, S. \& Balakrishnan, N. (1994a). Continuous Univariate Distributions, $2^{\mathrm{a}}$ ed., vol. 1. New York: John Wiley \& Sons. 11

Johnson, N. L., Kotz, S. \& Balakrishnan, N. (1994b). Continuous Univariate Distributions, $2^{\mathrm{a}}$ ed., vol. 2. New York: John Wiley \& Sons. 11

Joseph, L., Berger, R. D. \& BéLisle, P. (1997). Bayesian and mixed Bayesian/likelihood criteria for sample size determination. Statistics in Medicine 16, 769-781. 15

Joseph, L. \& BÉLISle, P. (1997). Bayesian sample size determination for normal means and differences between normal means. Journal of the Royal Statistical Society: Series D (The Statistician) 46, 209-226. 16, 26, 27

Joseph, L. \& Wolfson, D. B. (1997). Interval-based versus decision theoretic criteria for the choice of sample size. Journal of the Royal Statistical Society: Series D (The Statistician) 46, 145-149. 16

Joseph, L., Wolfson, D. B. \& Berger, R. D. (1995). Sample size calculations for binomial proportions via highest posterior density intervals. Journal of the Royal Statistical Society: Series $D$ (The Statistician) 44, 143-154. 15, 27, 40

Kalli, M., Griffin, J. E. \& Walker, S. G. (2011). Slice sampling mixture models. Statistics and Computing 21, 93-105. 55 
Lindley, D. V. (1997). The choice of sample size. Journal of the Royal Statistical Society: Series $D$ (The Statistician) 46, 129-138. 16, 19, 32

LiU, T.-K., Chang, C.-H. \& Chou, M.-L. (2014). Management strategies to prevent the introduction of non-indigenous aquatic species in response to the Ballast Water Convention in Taiwan. Marine Policy 44, 187-195. 35

Lo, A. Y. (1984). On a class of Bayesian nonparametric estimates: I. Density estimates. The Annals of Statistics 12, 351-357. 54

MacEachern, S. N. \& Müller, P. (1998). Estimating mixture of Dirichlet process models. Journal of Computational and Graphical Statistics 7, 223-238. 55

Matuszewski, D. J., Cesar, R. M., Strickler, J. R., Baldasso, L. F. \& Lopes, R. M. (2015). Visual rhythm for particle analysis in sample-in-flow systems: application for continuous plankton monitoring. Limnology and Oceanography: Methods 13, 687-696. 35

McCarthy, S. A., McPhearson, R. M., Guarino, A. \& Gaines, J. (1992). Toxigenic Vibrio cholerae 01 and cargo ships entering Gulf of Mexico. Lancet 339, 624-625. 1

Miller, A. W., Frazier, M., Smith, G. E., Perry, E. S., Ruiz, G. M. \& Tamburri, M. N. (2011). Enumerating sparse organisms in ships' ballast water: why counting to 10 is not easy. Environmental Science \& Technology 45, 3539-3546. 1, 34

M'Lan, C. E., Joseph, L. \& Wolfson, D. B. (2006). Bayesian sample size determination for case-control studies. Journal of the American Statistical Association 101, 760-772. 16, 29, 40

M'Lan, C. E., Joseph, L. \& Wolfson, D. B. (2008). Bayesian sample size determination for binomial proportions. Bayesian Analysis 3, 269-296. 16, 17, 19, 29

Müller, P. \& Parmigiani, G. (1995). Optimal design via curve fitting of Monte Carlo experiments. Journal of the American Statistical Association 90, 1322-1330. 21

Murphy, K. R., Ritz, D. \& Hewitt, C. L. (2002). Heterogeneous zooplankton distribution in a ship's ballast tanks. Journal of Plankton Research 24, 729-734. 1, 3

Nassar, M. M., Khamis, S. M. \& Radwan, S. S. (2010). Geometric sample size determination in Bayesian analysis. Journal of Applied Statistics 37, 567-575. 16

Nassar, M. M., Khamis, S. M. \& Radwan, S. S. (2011). On Bayesian sample size determination. Journal of Applied Statistics 38, 1045-1054. 16

Papaspiliopoulos, O. \& Roberts, G. O. (2008). Retrospective Markov chain Monte Carlo methods for Dirichlet process hierarchical models. Biometrika 95, 169-186. 55

Parmigiani, G. \& Inoue, L. Y. T. (2009). Decision Theory: principles and approaches. New York: John Wiley \& Sons. 16, 19, 20, 23

Pham-Gia, T. \& Turkkan, N. (1992). Sample size determination in Bayesian analysis. Journal of the Royal Statistical Society: Series D (The Statistician) 41, 389-392. 16

R Core Team (2016). R: A Language and Environment for Statistical Computing. R Foundation for Statistical Computing, Vienna, Austria. URL https://www.R-project.org/. 4, 35

Rahme, E., Joseph, L. \& Gyorkos, T. W. (2000). Bayesian sample size determination for estimating binomial parameters from data subject to misclassification. Journal of the Royal Statistical Society: Series C (Applied Statistics) 49, 119-128. 16, 28 
Rice, K. M., Lumley, T. \& Szpiro, A. A. (2008). Trading bias for precision: decision theory for intervals and sets. http://www.bepress.com/uwbiostat/paper336. Working Paper 336, UW Biostatistics. 22, 23, 42, 43

Ruiz, G. M., Rawlings, T. K., Dobbs, F. C., Drake, L. A., Mullady, T., Huq, A. \& Colwell, R. R. (2000). Global spread of microorganisms by ships. Nature 408, 49-50. 1

Sahu, S. K. \& Sмith, T. M. F. (2006). A Bayesian method of sample size determination with practical applications. Journal of the Royal Statistical Society: Series A (Statistics in Society) 169, 235-253. 16

Schervish, M. (1995). Theory of Statistics. New York: Springer-Verlag. 23

Sen, P. K., Singer, J. M. \& De Lima, A. C. P. (2009). From Finite Sample to Asymptotic Methods in Statistics. Cambridge: Cambridge University Press. 40

Sethuraman, J. \& Tiwari, R. C. (1981). Convergence of Dirichlet measures and the interpretation of their parameter. Relatório técnico M583, Florida State University. DTIC Document. 31, 54

Souza, R. C. C. L., Fernandes, F. C., Danelon, O. M., Larsen, K. T. S., Silva, J. S. V., Collichio, F. \& Rapagnã, L. (2001). Metodologia de amostragem dos organismos transportados em água de lastro dos navios mercantes. Pesquisa Naval 14, 221-236. 1

Stamey, J. D., Young, D. M. \& Bratcher, T. L. (2006). Bayesian sample-size determination for one and two Poisson rate parameters with applications to quality control. Journal of Applied Statistics 33, 583-594. 16, 40

Tsolaki, E. \& Diamadopoulos, E. (2010). Technologies for ballast water treatment: a review. Journal of Chemical Technology and Biotechnology 85, 19-32. 35

Wang, F. \& Gelfand, A. E. (2002). A simulation-based approach to Bayesian sample size determination for performance under a given model and for separating models. Statistical Science 17, 193-208. 16 


\section{Índice Remissivo}

amostra representativa, 2

Bayes

decisão de, 20, 22-24, 56

risco de, 16, 20, 21, 56, 60

cólera, 1

critério

da cobertura média, 15, 16

do comprimento médio, 15, 18, 19

Dirichlet

distribuição, 15, 51, 52

mistura de processos, 14, 24, 56

mistura por processo, 14, 24, 56

processo, $7,13,14,23,24,51-53,55,56$

distribuição

base, 14, 53

beta, 16, 19, 52

beta-linha, 11

binomial, 1, 5, 15, 16, 19

binomial negativa, 1, 3-6, 11, 15, 18, 39, 49, 50,59

condicional completa, 55

Dirichlet, 15, 52

exponencial, 16

gama, 3, 4, 9, 16, 39

geométrica, 16

Laplace, 16

misturadora, 14

multinomial, 15

normal, 15, 16, 19

Pearson Tipo VI, 11, 47-50

Poisson, 1-6, 9, 10, 15, 16

qui-quadrado, 15

Weibull, 16

erro

de estimação absoluto, 5

de estimação relativo, 5

do Tipo I, 3, 15

do Tipo II, 3, 15

estudos caso-controle, 16 família exponencial, 16

função custo, 16

função de ponderação, 23

função perda

linex, 16

linex limitada, 16

logarítmica, 16

para teste de hipóteses, 16

quadrática, 16

gama

distribuição, 3, 4, 9, 16, 51

função, 3

método dos mínimos quadrados, 21, 22

mediana, 17

medida de probabilidade aleatória, 13, 14

moda, 17

modelos de sobrevivência, 16

multimodal, 18

organismos

nocivos, 1

vivos, 1,2

patógenos humanos, 1

procedimento sequencial, 6,35

regressão

linear, 16, 21

logística, 16

série de Taylor, 21

taxa de decrescimento, 21

urna de Pólya, 53, 55 